\title{
Stellar populations of early-type galaxies in different environments ${ }^{\star}$
}

\section{Line-strength indices. Relations of line-strengths with $\sigma$}

\author{
P. Sánchez-Blázquez ${ }^{1,2}$, J. Gorgas ${ }^{2}$, N. Cardiel ${ }^{2,3}$, and J. J. González ${ }^{4}$ \\ ${ }^{1}$ Laboratoire d'Astrophysique, École Polytechnique Fédérale de Lausanne (EPFL), Observatoire, 1290 Sauverny, Switzerland \\ e-mail: patricia.sanchezblazquez@epfl.ch \\ 2 Dpto. de Astrofísica, Fac. de Ciencias Físicas, Universidad Complutense de Madrid, 28040 Madrid, Spain \\ 3 Calar Alto Observatory, CAHA, Apartado 511, 04004 Almería, Spain \\ 4 Instituto de Astronomía, Universidad Nacional Autónoma de México, Apdo. Postal 70-264, México D.F., México
}

Received 11 January 2006 / Accepted 26 April 2006

\begin{abstract}
Aims. This paper commences a series devoted to the study of the stellar content of early-type galaxies. The goal of the series is to set constraints on the evolutionary status of these objects.

Methods. In this paper we describe the details of the galaxy sample, the observations, and the data reduction. Line-strength indices and velocity dispersions $(\sigma)$ are measured in 98 early-type galaxies drawn from different environments, and the relation of the indices with the velocity dispersion analysed in detail.

Results. The present sample indicates that some of the index $-\sigma$ relations depend on galaxy environment. In particular, the slope of the relation between Balmer lines and $\sigma$ is steeper for galaxies in the Virgo cluster, small groups, and in the field than for galaxies in the Coma cluster. In several indices there is also a significant offset in the zero point between the relations defined by the different subsamples. The slopes of the index $-\sigma$ relation for the Virgo and low-density environment galaxies are explained by a variation of both age and metallicity, with velocity dispersion, as previously noted in other studies. For the galaxies in the Coma cluster, however, the relation of the indices with $\sigma$ only requires a variation of the abundance along the $\sigma$ sequence. In agreement with other studies, we find that the models that better reproduce the slopes are those in which the $\alpha$ elements vary more than the Fe-peak elements along the $\sigma$ sequence, while, at a given $\sigma$, older galaxies show an higher $\alpha /$ Fe ratio.

Conclusions. The results can be explained assuming that galaxies in the Coma cluster have experienced a truncated star formation and chemical enrichment history compared to a more continuous time-extended history for their counterparts in lower density environments.
\end{abstract}

Key words. galaxies: evolution - galaxies: elliptical and lenticular, cD - galaxies: stellar content

\section{Introduction}

There have long been two competing views on the star formation history of the elliptical galaxies in the present day Universe. The modern version of the classical "monolithic collapse" scenario puts the stress on elliptical assembly out of gaseous material (that is, with dissipation), in the form of either a unique cloud or many gaseous clumps, but not out of preexisting stars. In this scenario, the stars form at high $z$ and on short timescales relative to spiral galaxies (Chiosi \& Carraro 2002; Matteucci 2003). The competing "hierarchical" scenario (e.g., Toomre 1977; Kauffmann 1996; Somerville et al. 1999; de Lucia et al. 2006) propounds that galaxies form hierarchically through successive, non-dissipative, random mergers of subunits over a wide redshift range. The first scenario is favoured by the tight relations followed by the elliptical families, such as the Fundamental Plane (Djorgovski \& Davis 1987), the colour-magnitude, and the $\mathrm{Mg}_{2}-\sigma$ relationships (Bender et al. 1993; Jørgensen 1999; Kuntschner 2000). The second scenario is favoured by the wide range in the apparent age of their stellar

* Appendices A-D are only available in electronic form at http://www.edpsciences.org populations (González 1993, hereafter G93; Faber et al. 1995; Trager et al. 2000a; Terlevich \& Forbes 2002; Caldwell et al. 1993; Denicolo et al. 2005), the kinematical and dynamical peculiarities (e.g., de Zeeuw et al. 2002), and the presence of shells and ripples, indicative of recent interactions, in a large number of elliptical galaxies (Schweizer et al. 1990).

A natural outcome of the hierarchical scenarios is that haloes in regions of the Universe that are destined to form a cluster collapse earlier and merge more rapidly (e.g., Kauffmann \& Charlot 1998; de Lucia et al. 2006). Therefore, the study of the stellar content of early-type galaxies in different environments should allow us to test the hierarchical clustering scenarios of early-type galaxy formation.

Several works have analysed the differences in the evolution of cluster and field early-type galaxies through the study of the Fundamental Plane (FP), but the results remain controversial. In general, the evolution of the trends in cluster galaxies suggests an earlier formation for these systems when compared with their analogs in the field (Treu et al. 1999, 2001, 2002; van Dokkum \& Ellis 2003; Gebhardt et al. 2003; Rusin et al. 2003; Bernardi et al. 2003; Yi et al. 2005), but the environmental dependencies do not appear to be as large (van Dokkum et al. 2001) as 
predicted by some interpretations of hierarchical models (e.g., Diaferio et al. 2001; de Lucia et al. 2004). However, the evolutionary trends in the FP can be hidden due to the age-metallicity degeneracy (Worthey 1994), if there is a relation between the age and the metallicity of the galaxies (Coles et al. 1999; Ferreras et al. 1999).

Another approach, in principle less affected by this problem, is to compare the absorption spectral features between galaxies in different environments.

Kuntschner \& Davies (1998) studied a sample of galaxies in the Fornax cluster finding that they are mostly coeval, which contrasts with other studies in which intermediate-age or young populations have been found in a large fraction of non-cluster luminous elliptical galaxies (e.g., Rose 1985; G93; Forbes et al. 1998; Trager et al. 2000; Caldwell et al. 2003; Denicolo et al. 2005). However, whether such contrasting results are a product of differences in the environment is still an unanswered question. Some authors claim that other parameters such as the luminosity or the morphological type determine the star formation history (SFH) of early-type galaxies rather than environment. In this context, the differences between cluster and field galaxies found in several studies could be due to dissimilarities in the luminosity range of the different samples (see Poggianti et al. 2001b) and/or different proportions of S0 with respect to E galaxies. For example, Kuntschner \& Davies (1998) found that, in the Fornax cluster, luminous elliptical galaxies are old and coeval, while (less luminous) S0 galaxies display a significant age spread. In accordance with this result, Smail et al. (2001) found, studying a sample of galaxies drawn from Abell 2218 at $z=0.17$, that elliptical at all magnitudes and luminous S0's are coeval, while the faintest S0's have younger luminosity-weighted ages (see also Treu et al. 2005).

Bernardi et al. (1998) found a small offset (0.007 \pm $0.002 \mathrm{mag}$ ) in the $\mathrm{Mg}_{2}-\sigma$ relation between cluster and field galaxies, but the same intrinsic scatter about the relation for both subsamples. They also concluded that the differences are mainly driven by the faint objects. These authors also studied the zero point of the $\mathrm{Mg}_{2}-\sigma$ relation among cluster early-type galaxies and did not find any dependence of this value with the cluster richness as measured by cluster X-ray luminosity, temperature of the ICM, or velocity dispersion of member galaxies.

More recently, Denicoló et al. (2005a) compared a sample of galaxies in groups and in the field with the sample of Fornax galaxies from Kuntschner (2000), finding that the slopes of the index $\sigma$ relations for ellipticals in low-density regions are not significantly different from those of cluster E/S0, although the scatter of the relations seems larger for group, field, and isolated ellipticals than for cluster galaxies. In the second paper of the series (Denicoló et al. 2005b), the authors calculate ages and metallicities, finding that elliptical galaxies in low density environments are, on average, 3-4 Gyr younger than their counterparts in the Fornax cluster. The only caveat in this study is that both samples only share one galaxy in common, and the differences can be due to systematic offsets in the individual indices.

Thomas et al. (2005) also carried out an study of 124 earlytype galaxies in high- and low-density environments. They also found, in agreement with Denicoló, that massive early-type galaxies in low density environments seem, on average, 2 Gyr younger and slightly $(\sim 0.05-0.1 \mathrm{dex})$ more metal rich than their counterparts in high-density environments, consisting of galaxies drawed from the Coma and Virgo clusters. Interestingly, these authors found very massive $\left(M>10^{11} M_{\odot}\right)$ S0 galaxies showing low average ages between 2 and $5 \mathrm{Gyr}$, and very high metallicities of $[\mathrm{Z} / \mathrm{H}] \leq 0.67 \mathrm{dex}$. These galaxies are only present in high density environments, contrasting the above quoted result by Smail et al. (2001).

The lack of conclusive evidence for or against systematic differences between cluster and field galaxies has prompted us to carry out a systematic study analysing the stellar population of galaxies in different environments.

This paper starts a series devoted to the study of the stellar population in local early-type galaxies. The goal of the series is to shed light on the star formation history of these systems and the influence of the environment on their evolution. Although there have been several studies with larger samples that have studied the stellar population of the early-type galaxies in the Coma cluster and in the field, this is the first time that a large number of spectral features are analysed in a homogenous and high signal-to-noise ratio sample containing galaxies in both environments. Furthermore, these features are compared with the new stellar population synthesis models of Vazdekis et al. (2006, in preparation; hereafter V06), which themselves are an updated version of the Vazdekis et al. (2003) models with an improved stellar library, MILES (Sánchez-Blázquez et al. 2006). The new library contains 1003 stars covering the atmospheric parameter space in a homogenous way, reducing the uncertainties of the predictions at metallicities departing from solar. Although a more detailed description of these models and the derivation of age and metallicities procedures will be given in the Paper II of this series, we discuss here some of the results based on them. We refer the reader to Vazdekis et al. (2003) for details regarding the ingredients of the models.

The outline of the paper is as follows: Sect. 2 presents the sample description, observations, and data reduction. Section 3 presents the absorption-line measurements. Section 4 shows the relation of the absorption line indices with the velocity dispersion of the galaxies. Section 5 summarises our conclusions.

\section{Observations and data reduction}

\subsection{Sample selection}

We analyse a sample of 98 early-type galaxies, which includes ellipticals (E) and S0 galaxies spanning a large range in velocity dispersion (from $40 \mathrm{~km} \mathrm{~s}^{-1}$ to $400 \mathrm{~km} \mathrm{~s}^{-1}$ ).

As one of the main goals of this work is to study the influence of the environment on the star formation history of early-type galaxies, the sample contains galaxies in the field, poor groups, and in the Virgo, Coma, and some Abell galaxy clusters. For the purpose of this series, we have divided the sample in two main groups that we call hereafter high density environment galaxies (HDEGs) and low density environment galaxies (LDEGs).

It is difficult to identify a single, optimal manner by which to separate a sample of galaxies to study the role of environment. In this series of papers, instead of using an indicator of the local density, we have chosen to separate and delineate galaxies by field, group, or cluster environments.

Therefore, in the first group we include galaxies residing in the central regions of the Coma cluster and one galaxy from the cluster Abell 2199 (NGC 6166). We have assigned the galaxies in the field, groups (including the groups Abell 569 and Abell 779 with very low values of velocity dispersion and X-ray luminosity (see, e.g., Xian-Ping \& Yan-Jie 1999)), and in the Virgo cluster to the second group. This assignment of Virgo galaxies to the LDEG group reflects that our classification should be taken in relative terms, HDEG meaning, in fact the Coma-like rich clusters, while the rest the of environments are assigned to the LDEG group. The Virgo cluster contains 
Table 1. Sample of galaxies in the different observing runs. Type: morphological type extracted from the NASA/IPAC Extragalactic Database; $M_{B}$ : absolute magnitude in the $B$-band, obtained from the Lyon-Meudon Extragalactic Database; Env.: when the galaxy is a member of a wellknown cluster, the cluster name is indicated; the label Pair indicates that the galaxy is a member of a pair of galaxies; when the galaxies do not belong to a cluster, group, or pair of galaxies, it is labeled as field galaxy; Run: observation run in which each galaxy was observed; $t_{\text {exp }}$ : exposure time (seconds); PA: position angle of the major axis of the galaxy measured from north to east (degrees); SA: orientation of the slit (degrees); Flag: environment assigned to each galaxy (L: low-density environment: H: high-density environment); Code: symbols used in Figure; Ref: reference used to assigned the environment Flag to each galaxy (Gc93: García 1993; VCC85: Binggeli et al. 1985; GMP: Godwin et al. 1983; CGCG: Zwicky et al. 1974). 6.

\begin{tabular}{|c|c|c|c|c|c|c|c|c|c|c|c|c|}
\hline$\overline{\overline{\text { Name }}}$ & RA (J2000) & $\overline{\text { Dec (J2000) }}$ & Type & $M_{\mathrm{B}}$ & Env. & Run & $t_{\exp }$ & PA & $\overline{\mathrm{SA}}$ & Flag & $\overline{\overline{C o d e}}$ & Ref. \\
\hline \multirow[t]{2}{*}{ NGC 221} & 004241.87 & +405157.2 & $\mathrm{cE} 2$ & -17.58 & LGG 11 & 1 & 1204 & 170 & 170.0 & $\mathrm{~L}$ & $\triangle$ & Gc93 \\
\hline & & & & & & 3 & 1200 & 170 & 0.0 & & & \\
\hline \multirow[t]{2}{*}{ NGC 315} & 005748.88 & +302108.8 & $E+$ & -22.37 & LGG 18 & 1 & 1800 & 40.0 & 0.0 & $\mathrm{~L}$ & 0 & Gc93 \\
\hline & & & & & & 3 & 2400 & 40.0 & 45.0 & & & \\
\hline NGC 507 & 012340.00 & +331521.9 & $\mathrm{SA}(\mathrm{r})$ & -22.15 & LGG 26 & 1 & 1585 & 67.0 & 0.0 & $\mathrm{~L}$ & 口 & Gc93 \\
\hline NGC 584 & 013120.72 & -065206.1 & E4 & -20.63 & NGC 584 group & 1 & 1800 & 120.0 & 0.0 & $\mathrm{~L}$ & 0 & Gc93 \\
\hline NGC 636 & 013906.52 & -073045.6 & E3 & -19.65 & LGG 27 & 1 & 1800 & 140.0 & 0.0 & $\mathrm{~L}$ & 0 & Gc93 \\
\hline NGC 821 & 020821.04 & +105941.1 & E6 & -20.57 & Field & 1 & 1200 & 25.0 & 0.0 & $\mathrm{~L}$ & 0 & \\
\hline NGC 1453 & 034627.22 & -035808.9 & E2-3 & -21.52 & LGG 103 & 1 & 1800 & 8.0 & 0.0 & $\mathrm{~L}$ & 0 & Gc93 \\
\hline \multirow[t]{2}{*}{ NGC 1600} & 043139.89 & -050510.1 & E3 & -22.31 & Field & 1 & 1800 & 170.0 & 0.0 & $\mathrm{~L}$ & 0 & \\
\hline & & & & & & 2 & 1800 & 170.0 & 195.0 & & & \\
\hline NGC 1700 & 045656.30 & -045152.0 & E4 & -21.80 & LGG 123 & 1 & 3000 & 65.0 & 0.0 & $\mathrm{~L}$ & 0 & Gc93 \\
\hline NGC 2300 & 073221.82 & +854232.2 & E-S0 & -20.85 & Abell 569 & 1 & 1500 & 78.0 & 0.0 & $\mathrm{~L}$ & $\square$ & Gc93 \\
\hline NGC 2329 & 070908.08 & +483653.3 & S0- & -21.73 & Abell 569 & 2 & 1800 & 175.0 & 175.0 & $\mathrm{~L}$ & 口 & Gc93 \\
\hline NGC 2693 & 085659.28 & +512049.5 & E3 & -21.67 & LGG 168 & 1 & 3200 & 160.0 & 0.0 & $\mathrm{~L}$ & 0 & Gc93 \\
\hline NGC 2694 & 085659.28 & +511955.1 & E1 & -19.15 & LGG 168 & 1 & 3200 & & 0.0 & $\mathrm{~L}$ & 0 & Gc93 \\
\hline NGC 2778 & 091224.35 & +350139.4 & $\mathrm{E}$ & -19.06 & LGG 171 & 2 & 1800 & 40.0 & 220.0 & $\mathrm{~L}$ & 0 & Gc93 \\
\hline NGC 2832 & 091946.89 & +334500.0 & $\mathrm{E}+2$ & -22.38 & Abell 779 & 2 & 1800 & 160.0 & 160.0 & $\mathrm{~L}$ & 0 & CGCG \\
\hline NGC 3115 & 100513.80 & -074308.0 & S0- & -19.77 & Field & 2 & 1500 & 43.0 & 310.0 & $\mathrm{~L}$ & 0 & \\
\hline NGC 3377 & 104742.36 & +135908.8 & E5-6 & -19.16 & Leo group (LGG217) & 1 & 1800 & 35.0 & 0.0 & $\mathrm{~L}$ & 0 & Gc93 \\
\hline \multirow[t]{2}{*}{ NGC 3379} & 104749.75 & +123454.6 & E1 & -20.57 & Leo group (LGG 217) & 1 & 900 & 71.0 & 0.0 & $\mathrm{~L}$ & 0 & Gc93 \\
\hline & & & & & & 2 & 1200 & 71.0 & 251.0 & & & \\
\hline NGC 3605 & 111646.69 & +180101.0 & E4-5 & -17.07 & LGG 237 & 2 & 1800 & 17.0 & 17.0 & $\mathrm{~L}$ & $\Delta$ & Gc93 \\
\hline NGC 3608 & 111659.07 & +180854.6 & E2 & -19.62 & LGG 237 & 1 & 1800 & 75.0 & 0.0 & $\mathrm{~L}$ & $\bigcirc$ & Gc93 \\
\hline NGC 3641 & 112108.85 & +03 1140.2 & E pec & -17.91 & LGG 233 & 2 & 1800 & 3.0 & 240.0 & $\mathrm{~L}$ & $\Delta$ & Gc93 \\
\hline NGC 3665 & 112443.64 & +384545.0 & SA(s) & -20.84 & LGG 236 & 1 & 1800 & 30.0 & 0.0 & $\mathrm{~L}$ & $\square$ & Gc93 \\
\hline NGC 3818 & 114157.50 & -060920.0 & E5 & -19.11 & Field & 2 & 1800 & 103.0 & 280.0 & $\mathrm{~L}$ & 0 & \\
\hline NGC 4261 & 121923.21 & +05 4929.7 & E2-3 & -21.32 & LGG 278 & 2 & 1800 & 160.0 & 160.0 & $\mathrm{~L}$ & 0 & Gc93 \\
\hline NGC 4278 & 122006.83 & +291650.7 & E1-2 & -19.26 & LGG 279 & 2 & 1800 & 22.7 & 22.0 & $\mathrm{~L}$ & 0 & Gc93 \\
\hline NGC 4365 & 122428.34 & +07 1904.2 & E3 & -20.90 & Virgo (LGG 289) & 2 & 1200 & 40.0 & 220.0 & $\mathrm{~L}$ & 0 & Gc93 \\
\hline NGC 4374 & 122503.74 & +125313.1 & E1 & -20.87 & Virgo (LGG 292) & 1 & 1800 & 135.0 & 0.0 & $\mathrm{~L}$ & 0 & Gc93 \\
\hline
\end{tabular}

significant substructure (e.g., de Vaucouleurs 1961; Binggeli et al. 1985; Binggeli et al. 1993; Gavazzi et al. 1999), being an aggregation of sub-clumps that are very likely in the process of merging (Binggeli 1997). The clump containing M 87 has an estimated mass from the X-ray haloes of $10^{14} M_{\odot}$, but the other main sub-clumps, which include M 86 and M49, have masses one order of magnitude less (Bohringer et al. 1994), which is the typical mass for a group of galaxies. All this suggests that Virgo can be considered as a group of groups rather than as a normal cluster. Furthermore, and admittedly, this is an adhoc argument, as the analysis of the stellar populations of Virgo early-type galaxies indicate that they are not dissimilar to those galaxies in poorer environments (field and small groups) (e.g., Concannon 2003; Sánchez-Blázquez 2004), while they are markedly different from our sample of ellipticals in the Coma cluster (as it will be shown throughout this paper). We decided to group the Virgo galaxies together with the rest of the galaxies in low-density environments to maximize the statistical significance of the sample. Galaxies were considered to be in a group if they were included in the Lyon Group of Galaxies catalogue (García 1993). This catalogue classifies galaxies in the Virgo cluster as belonging to the groups LGG 289 and LGG 292. Galaxies were considered to be "Field" galaxies if they were not listed as a member of a Lyon Galaxy Group catalogue or as a member of a known cluster. Coma clusters were selected from both the GMP catalogue (Godwin et al. 1983) and the catalogue of galaxies and clusters of galaxies (Zwicky et al. 1961). The last column in Table 1 lists the catalogue reference giving the group assigment. Galaxies in both subsamples were selected to span a wide range of luminosity and observational properties. Table 1 lists the whole sample together with their morphological type and absolute magnitude. The distribution in velocity dispersion for LDEGs and HDEGs is shown in Fig. 1. Although the $\sigma$ range covered by both subsamples is similar, the LDEG sample is slightly skewed towards more massive objects.

\subsection{Observations}

Long-slit spectroscopy was carried out in four observing runs using two different telescopes. In Runs 1 and 3 (January 1998 and August 1999), we used the Cassegrain Twin Spectrograph with a blue coated TEK CCD in the blue channel on the $3.5 \mathrm{~m}$ telescope at the German-Spanish Astronomical Observatory at Calar Alto (Almería, Spain). The observations in Runs 2 and 4 (March 1999 and April 2001) were carried out with the ISIS double spectrograph mounted at the f/11 Cassegrain focus on the William Herschel Telescope in the Roque de los Muchachos Observatory (La Palma, Spain). Details of the observational 
Table 1. continued.

\begin{tabular}{|c|c|c|c|c|c|c|c|c|c|c|c|c|}
\hline Name & RA (J2000) & $\overline{\mathrm{DEC}(\mathrm{J} 2000)}$ & Type & $\overline{M_{\mathrm{B}}}$ & Env. & Run & $\overline{t_{\exp }}$ & $\overline{\mathrm{PA}}$ & $\overline{\mathrm{SA}}$ & $\overline{\text { Env }}$ & Code & $\overline{\text { Ref. }}$ \\
\hline \multirow[t]{2}{*}{ NGC 4415 } & \multirow{2}{*}{122640.56} & \multirow{2}{*}{+082607.5} & \multirow{2}{*}{ S0/a } & \multirow{2}{*}{-17.27} & \multirow[t]{2}{*}{ Virgo (LGG 292) } & 1 & 4200 & 0.0 & $\overline{0.0}$ & \multirow{2}{*}{$\mathrm{L}$} & \multirow[t]{2}{*}{$\Delta$} & \multirow[t]{2}{*}{ Gc93 } \\
\hline & & & & & & 2 & 3000 & 0.0 & 180.0 & & & \\
\hline JGC 4431 & 122727.43 & +121723.8 & $\mathrm{SA}(\mathrm{r}) 0$ & -16.95 & Virgo (LGG 292) & 1 & 7200 & 177.0 & 0.0 & $\mathrm{~L}$ & $\triangle$ & Gc93 \\
\hline NGC 4464 & 122921.38 & +080923.1 & E3 & -18.12 & Virgo (LGG 289) & 2 & 1800 & 0.0 & 180.0 & $\mathrm{~L}$ & $\square$ & Gc93 \\
\hline NGC 4467 & 122930.35 & +075938.3 & E2 & -16.89 & Virgo (LGG 289) & 2 & 1800 & 81.2 & 99.0 & $\mathrm{~L}$ & 0 & Gc93 \\
\hline NGC 4472 & 122946.76 & +075959.9 & $\mathrm{E} 2 / \mathrm{SO}(2)$ & -21.47 & Virgo (LGG 292) & 2 & 1200 & 155.0 & 155.0 & $\mathrm{~L}$ & 口 & Gc93 \\
\hline NGC 4478 & 123017.53 & +121940.3 & E2 & -19.55 & Virgo (LGG 289) & 2 & 1800 & 140.0 & 140.0 & $\mathrm{~L}$ & 0 & Gc93 \\
\hline NGC 4486B & 123031.82 & +122925.9 & cE0 & -17.46 & Virgo (LGG 289) & 1 & 1800 & 117.3 & 0.0 & $\mathrm{~L}$ & $\bigcirc$ & Gc93 \\
\hline NGC 4489 & 123052.34 & +164530.4 & $\mathrm{E}$ & -18.25 & Virgo (LGG 292) & 1 & 2500 & & 0.0 & $\mathrm{~L}$ & $\triangle$ & Gc93 \\
\hline GC 4552 & 123 & +123322.9 & E & -20.91 & Virgo & 1 & 1800 & & 0.0 & $\mathrm{~L}$ & 0 & VCC85 \\
\hline GC 4564 & 1236 & +112618.8 & E6 & -19.43 & Virgo (LGC & 2 & 1500 & 47.0 & 47.0 & $\mathrm{~L}$ & $\bigcirc$ & Gc93 \\
\hline GC 4594 & 123 & -113 & $\mathrm{SA}(\mathrm{s}) \mathrm{a}$ & -22.36 & Field & ? & 1013 & 89.0 & 179.0 & $\mathrm{~L}$ & 0 & \\
\hline GC 4621 & 124202.39 & +113845.1 & E5 & -20.71 & Virgo & 2 & 1200 & 165.0 & 165.0 & $\mathrm{~L}$ & 0 & VCC85 \\
\hline GC 4636 & 124250.00 & +024116.5 & E-S0 & -20.78 & Field & 1 & 1200 & 150.0 & 0.0 & $\mathrm{~L}$ & $\square$ & \\
\hline GC 4649 & 124340.19 & +113308.9 & E2 & -21.53 & Virgo (LGG 292) & 1 & 900 & 105.0 & 0.0 & $\mathrm{~L}$ & $\bigcirc$ & Gc93 \\
\hline NGC 4673 & 124534.77 & +270337.3 & E1-2 & -20.52 & Coma & 4 & 1800 & 170.0 & 170.0 & $\mathrm{H}$ & 0 & GMP \\
\hline NGC 4692 & 124755.42 & +271318.3 & $\mathrm{E}+$ & -21.70 & Coma & 4 & 1500 & 97.0 & 97.0 & $\mathrm{H}$ & 0 & GMP \\
\hline NGC 4697 & 124835.70 & -054803.0 & E6 & -20.97 & LGG 314 & 2 & 1200 & 70.0 & 70.0 & $\mathrm{~L}$ & 0 & Gc93 \\
\hline NGC 4742 & 125147.92 & -102717.1 & E4 & -22.40 & LGG 307 & 2 & 1800 & 75.0 & 75.0 & $\mathrm{~L}$ & 0 & Gc93 \\
\hline NGC 4839 & 125724.31 & +272952.0 & $\mathrm{cD} ; \mathrm{SA} 0$ & -21.84 & Coma & 4 & 5400 & 65.0 & 49.4 & $\mathrm{H}$ & $\square$ & GMP \\
\hline NGC 4842A & 125735.60 & +272 & E/SA0 & -19.89 & Coma & 4 & 1800 & & 172.4 & $\mathrm{H}$ & $\square$ & GMP \\
\hline NGC 4842B & 125736.14 & 05.6 & SA0 & -18.80 & Coma & 4 & 1800 & & 172.4 & $\mathrm{H}$ & $\square$ & GMP \\
\hline NGC 4864 & 125 & +275 & E2 & -20.55 & Coma & 4 & 1800 & & 128.7 & $\mathrm{H}$ & 0 & GMP \\
\hline JGC & 125 & +28 & $\mathrm{E}$ & -19.91 & $\mathrm{Co}$ & 4 & 1800 & & 70.0 & $\mathrm{H}$ & 0 & GMP \\
\hline NGC 4 & 125 & +275 & E3 & -19.01 & $\mathrm{Co}$ & 4 & 1800 & & 128.7 & $\mathrm{H}$ & $\bigcirc$ & GMP \\
\hline NGC 4874 & 125935.91 & +275730.8 & $\mathrm{cD}$ & -22.53 & Coma & 2 & 1800 & 39.7 & 79.0 & $\mathrm{H}$ & $\bigcirc$ & GMP \\
\hline & & & & & $\mathrm{Co}$ & 4 & 3600 & 39.7 & 39.7 & & & \\
\hline GC 4875 & 1259 & +275 & SAB0 & -19.72 & Coma & 4 & 1800 & & 48.5 & $\mathrm{H}$ & $\square$ & GMP \\
\hline NGC 4889 & 130008.03 & +275835.1 & $\mathrm{cD}$ & -22.46 & Coma & 2 & 2178 & 80.0 & 80.0 & $\mathrm{H}$ & 0 & GMP \\
\hline & & & & & & 4 & 3800 & 80.0 & 80.0 & & & \\
\hline JGC 4908 & 1300 & +280233.6 & E5 & -21.01 & Coma & 4 & 1800 & & 163.0 & $\mathrm{H}$ & 口 & GMP \\
\hline NGC 5638 & 142940.30 & +031400.0 & E1 & -19.78 & LGG 386 & 2 & 1800 & 150.0 & 150.0 & $\mathrm{~L}$ & $\bigcirc$ & Gc93 \\
\hline NGC 5796 & 145924.20 & -163724.0 & E0-1 & -20.77 & LGG 386 & 2 & 1800 & 95.0 & 194.0 & $\mathrm{~L}$ & $\bigcirc$ & Gc93 \\
\hline NGC 5812 & 150055.60 & -072726.0 & E0 & -20.36 & Field & 2 & 1800 & 130.0 & 130.0 & $\mathrm{~L}$ & $\bigcirc$ & \\
\hline NGC 5813 & 150111.32 & +014206.4 & E1-2 & -20.99 & LGG 393 & 2 & 1800 & 145.0 & 145.0 & $\mathrm{~L}$ & $\bigcirc$ & Gc93 \\
\hline NGC 5831 & 150407.10 & +01 1311.7 & E3 & -19.72 & LGG 393 & 2 & 1800 & 55.0 & 235.0 & $\mathrm{~L}$ & 0 & Gc93 \\
\hline NGC 5845 & 150600.90 & +013801.4 & $\mathrm{E}$ & -18.58 & LGG 392 & 2 & 1800 & 150.0 & 150.0 & $\mathrm{~L}$ & 0 & Gc93 \\
\hline NGC 5846 & 150629.37 & +013619.0 & E0-1 & -21.30 & LGG 393 & 2 & 1200 & & 182.0 & $\mathrm{~L}$ & 0 & Gc93 \\
\hline & & & & & & 4 & 1000 & & 182.0 & & & \\
\hline
\end{tabular}

configurations for each run are given in Table 2. As can be seen, the spectral resolution of Run 4 is very different from the rest of the runs. As the Lick indices depend on the instrumental broadening (as well as velocity dispersion broadening), special care has to be taken to degrade the spectra to the Lick resolution to avoid systematic differences between observing Runs. Typical exposure times varied from $1800 \mathrm{~s}$ for the brightest galaxies to $7200 \mathrm{~s}$ for the faintest ones in the Coma cluster. Typical signalto-noise ratios per $\AA$, measured in the range between 3500 and $6500 \AA$, are 110 and 50 for the LDEGs and HDEGs galaxies, respectively. The wavelength coverage varies between different runs, but all includes the range between 3500 and $5250 \AA$, which allows us to the measure the D4000 break (Bruzual 1983) and $15 \mathrm{Lick} / \mathrm{IDS}$ indices (from $\mathrm{H} \delta_{A}$ to $\mathrm{Mgb}$; Trager et al. 1998, hereafter T98). When possible, the slit was oriented along the major axis of the galaxies. The high quality of the data allowed us to extract spatially resolved spectra and measured the indices out to the effective radius with a high signal-to-noise ratio. In this paper, we analyse only the central regions of the galaxies. The analysis of the gradients will be the objective of Paper III in this series.

Table 1 lists the sample of galaxies together with additional information including total exposure times and position angles of the spectrograph slit. Additionally, we observed about $45 \mathrm{G}-\mathrm{K}$ stars to be used as templates for velocity dispersion measurements, as well as to transform our line-strength indices to the Lick system. Flux standards from Oke (1990) were observed to correct the continuum shape of the spectra. To check for the possibility of systematic effects between different runs, several galaxies were observed in more than one observing period.

\subsection{Data reduction}

The standard data reduction procedures (flat-fielding, cosmic ray removal, wavelength calibration, sky subtraction, and fluxing) were performed with $\mathrm{R}_{\mathrm{E}} \mathrm{D}_{\mathrm{m}}^{\mathrm{uC}} \mathrm{E}$ (Cardiel 1999). This reduction package allows a parallel treatment of data and error frames and, therefore, produces an associated error spectrum for each individual data spectrum. We want to stress the importance of obtaining reliable errors on the measurements of individual features to analyse the effects of the correlated errors between the derived stellar population parameters. The use of this package allowed us to control errors more easily than what would have been possible with other available software packages. 
Table 1. continued.

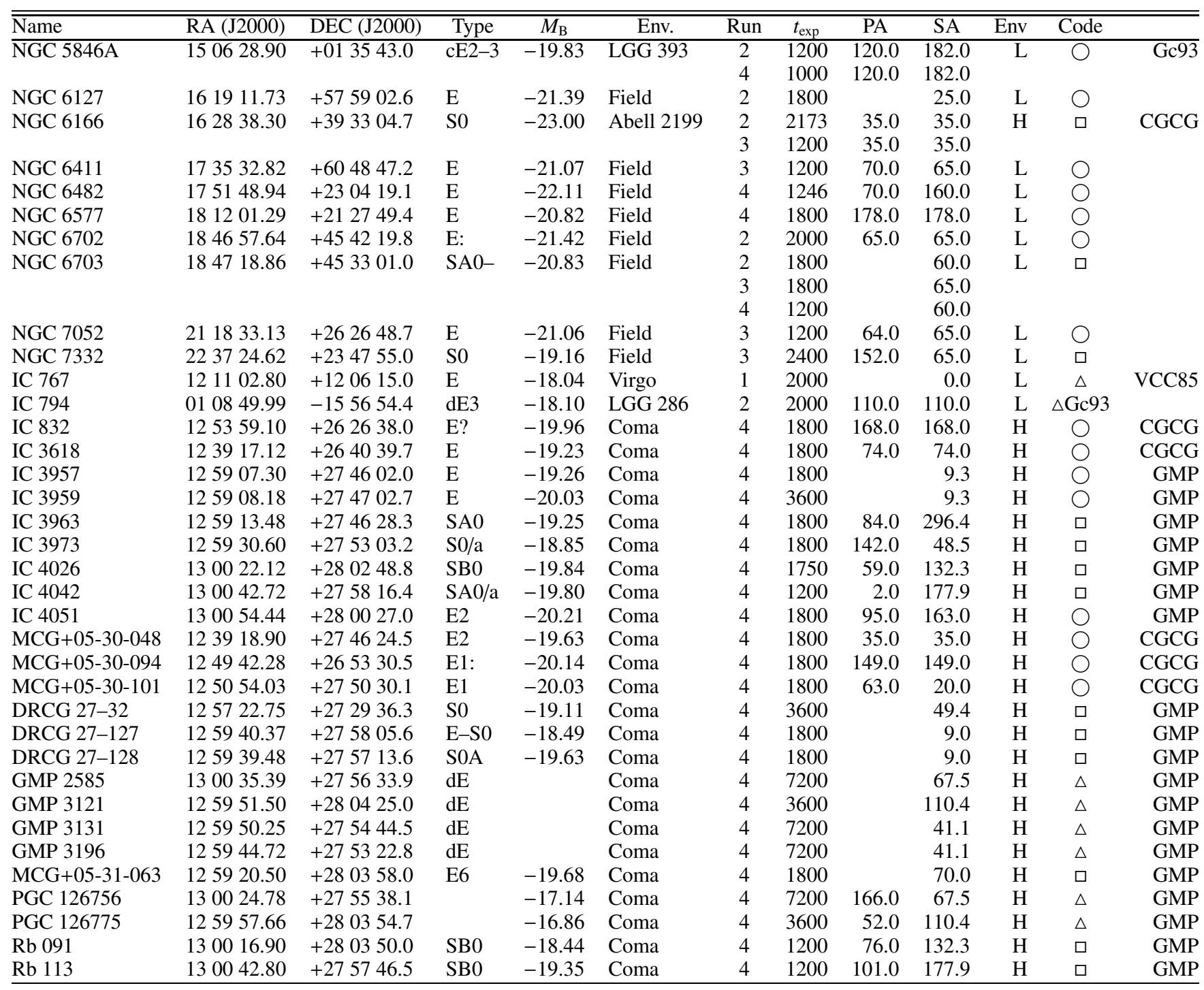

Initial reduction of the CCD frames involved bias and dark current subtraction, the removal of pixel-to-pixel sensitivity variations (using flat-field exposures of a tungsten calibration lamp), and correction for two-dimensional low-frequency scale sensitivity variations (using twilight sky exposures).

The dichroics in Runs 2 and 4 produced an intermediate frequency pattern that varies with the position of the telescope. This pattern was removed during flat-fielding in the images of Run 4. Unfortunately, in Run 2 this was not possible as we did not acquire flat field exposures in all the galaxy positions. Fortunately, several galaxies from Run 2 were also observed in other runs. By dividing the spectra of these galaxies from Run 2 by the spectra acquired in other runs (previously resampled to the same instrumental resolution and dispersion), we obtained the shape of the oscillations in Run 2. The shape of this pattern turned out to be identical for all the galaxies with repeated observations, with the exception of small offsets between them. These offsets were quantified with respect to a galaxy of reference for all the galaxies of Run 2 (given the characteristic shape of the oscillation pattern, even for galaxies without repeated observations, it was straightforward to determine this offset using, for this purpose, similar spectra from other observing runs). Then, all the galaxies were divided by this reference pattern shifted to the previously calculated offset (for further details of the procedure, see Sánchez-Blázquez 2004). The uncertainty introduced by this correction was added to the final error budget, although it is not a dominant error source (see Table 4).

Prior to the wavelength calibration, arc frames were used to correct from $\mathrm{C}$-distortion in the images. This rotation correction guaranteed alignment errors to be below 0.1 pixel. Spectra were converted to a linear wavelength scale using typically 120 arc lines fitted by 3 th-5th order polynomials, with rms errors of $0.3-0.6 \AA$. In addition, all the spectra were corrected for S-distortion. This correction is performed with a routine that finds the maximum corresponding to the center of the galaxy as a function of wavelength and fits these positions with a low-order polynomial. Finally, the spectra were displaced with a technique that minimizes the errors due to the discretization of the signal. This technique does not assume that the signal is constant in a given pixel, but adopts a more realistic distribution by fitting a second order polynomial using the available information in the adjacent pixels. The S-distortion can change along the slit, but the correction only affect the measurements in the central parts, where the signal gradient is important. Therefore, we assume 


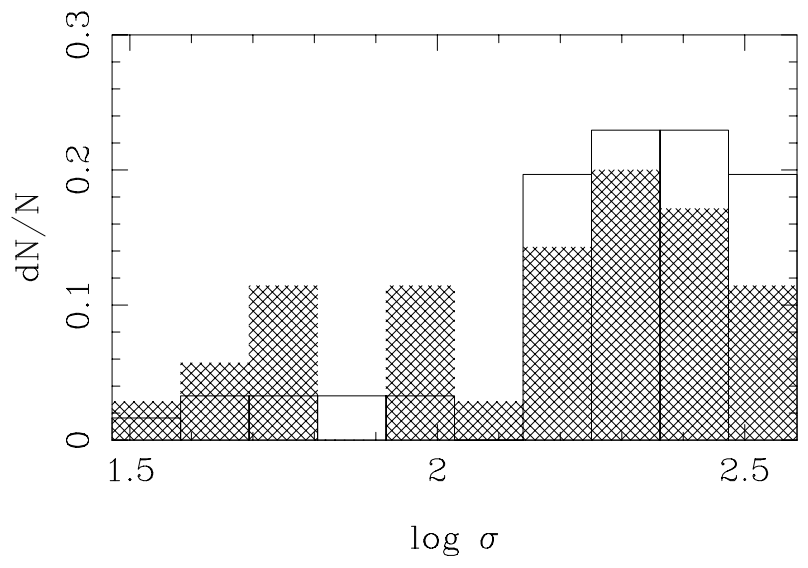

Fig. 1. Distribution of central velocity dispersion in HDEGs (grey shaded histogram) and LDEGs (empty histogram). The mean values of the distributions are $2.27 \pm 0.24$ and $2.16 \pm 0.30$ for LDEGs and HDEGs, respectively. The probability that the differences are due to chance is $\sim 50 \%$.

that the error of using the S-distortion pattern derived from the central galaxy region in the whole extent of the slit can be safely ignored.

Atmospheric extinction was calculated using the extinction curve of King (1985) for Runs 2 and 4, and the extinction curve of Calar Alto for Runs 1 and 3. To correct the effect of interstellar extinction, we used the curve of Savage \& Mathis (1979). The reddenings were extracted from the RC3 catalogue of galaxies (de Vaucouleurs et al. 1991). Relative flux calibration of the spectra was achieved using exposures of standard stars (3, 4, 5, and 33 exposures were taken in Runs 1, 2, 3, and 4, respectively). All the calibration curves of each run were averaged and the flux calibration errors were estimated by the differences between the indices measured with different curves. To transform our indices into the spectrophotometric system of Lick/IDS, we measured line strengths in a sample of G-K giant stars in common with Worthey et al. (1994). The comparison is presented in Appendix A.

Since in the outer parts of some galaxies we measure indices in spectra with light levels corresponding to only a few per cent of the sky signal (Paper III), the sky subtraction is a critical step in our data reduction to obtain reliable gradients. After correcting for both C-and S-distortion, a sky image was generated for each galaxy observation. This was done by fitting a low-order polynomial (order zero or one) to regions selected at both sides of the galaxy for each channel (pixel in the $\lambda$ direction). A possible systematic overestimation of the sky level could arise if the galaxy contribution to the regions from where the sky is extracted was not negligible. This overestimation of the sky level could increase the measured indices dramatically in the outer regions. To explore this effect we have fitted de Vaucouleurs profiles to the surface brightness profiles of our galaxies to estimate the relative contribution of the galaxy. When necessary, the effect has been taken into account, subtracting a scaled and averaged galaxy spectrum from the sky spectra. An underestimation of the sky level is very unlikely because this would imply high systematic errors that should be clearly detected such as unremoved sky lines (see Cardiel et al. 1995).

From each fully reduced galaxy frame, the spectra within an equivalent aperture of $4^{\prime \prime}$ at a redshift $z=0.016$ were coadded. This corresponds to a physical aperture size of $0.62 \mathrm{kpc}$ assuming $H_{0}=70 \mathrm{~km} \mathrm{~s}^{-1} \mathrm{Mpc}^{-1}$. This aperture was chosen as a compromise to obtain a fair number of co-added spectra for galaxies in the Coma and in the Virgo clusters.
This method does not fully extract the same physical area for all galaxies, as the slit width was kept constant. To quantify this second-order aperture effect, we also extracted spectra along the slit, simulating equal-physical-area circular apertures (distance-weighted co-added spectra), and found no significant differences. Therefore, we chose to work with the first aperture (without correcting for second-order effects) so as to maximize the $\mathrm{S} / \mathrm{N}$ of our spectra.

\section{Velocity dispersion measurements}

Radial velocities and velocity dispersions for the galaxies were measured using the MOVEL and OPTEMA algorithms described by G93. The MOVEL algorithm is an iterative procedure based in the Fourier Quotient method (Sargent et al. 1977) in which a galaxy model is processed in parallel to the galaxy spectrum. The main improvement of the procedure is introduced through the OPTEMA algorithm, which is able to overcome the typical template mismatch problem by constructing an optimal template as a linear combination of stellar spectra of different spectral types and luminosity classes for each galaxy (see, for details, G93; Pedraz et al. 2002; Sánchez-Blázquez 2004). To build the optimal template, we made use of 25,40 , 10 , and 33 stars in Runs $1,2,3$, and 4 respectively. To illustrate the procedure, Fig. 2 shows a typical fit between the observed central spectrum of a galaxy and the corresponding optimal template corrected with the derived kinematic parameters. The errors in the radial velocity and velocity dispersion $(\sigma)$ were computed through numerical simulations. In each simulation, a bootstrapped galaxy spectrum, obtained using the error spectrum provided by the reduction with $\mathrm{RED}_{\mathrm{m}}^{\mathrm{uC}} \mathrm{E}$, is fed into the MOVEL and OPTEMA algorithms (note that a different optimal template is computed in each simulation). Errors in the parameters were then calculated as the unbiased standard deviation of the different solutions. These final errors are expected to be quite realistic, as they incorporate all the uncertainties of the whole reduction process, from the first steps (e.g., flat-fielding) to the final measurements of the parameters.

To check the quality of the measured kinematics, we compared our derived velocity dispersions with different data compiled from the literature. We have chosen four different studies: G93, T98, Kuntschner et al. (2001), and Moore et al. (2002), with $33,52,31$, and 12 galaxies in common with our sample, respectively. Figure 3 shows the result of this comparison and, in Table 3, we summarise the mean differences. As can be seen, our velocity dispersions are, on average, $\sim 15 \mathrm{~km} \mathrm{~s}^{-1}$ larger than those in other studies. In particular, the differences seem to be larger as $\sigma$ increases. Using a linear fit to the data (represented in Fig. 3, we calculated that the maximum difference, for a galaxy with $\sigma=400 \mathrm{~km}^{-1}$, is $17 \mathrm{~km} \mathrm{~s}^{-1}$. This difference in $\sigma$ translates into errors in the indices of less than $0.1 \%$. This systematic effect can be the consequence of template mismatches either in our study or in the others. Nevertheless, our method makes use of several stars to calculate the optimal template, then minimises the effect of a poor fit. Furthermore, the effect of mismatching tend to underestimate the values of the velocity dispersion (Laird \& Levison 1985; Bender 1990), instead of making them larger. It is not the purpose of this section to investigate the real causes of the differences as this systematic offset does not affect any of our conclusions.

\section{Line-strength indices}

Line-strength indices in the Lick/IDS system (e.g., T98) and the D4000 defined by Bruzual (1983) were measured in these 
Table 2. Observational configurations employed in the four different observing runs.

\begin{tabular}{lllll}
\hline \hline & Run 1 & Run 2 & Run 3 & Run 4 \\
\hline Dates & 19-21 Jan. 1998 & 15-17 Mar. 1999 & 18-19 Aug. 1999 & 25-27 Apr. 2001 \\
Telescope & CAHA 3.5 m & WHT 4.2 m & CAHA 3.5 m & WHT 4.2 m \\
Spectrograph & CTS & ISIS blue & CTS & ISIS blue \\
Detector & CCD TEK 12 & EEV12 & CCD TEK 12 & EEV12 \\
Dispersion & $1.08 \AA /$ pixel & $0.80 \AA /$ pixel & $1.08 \AA /$ pixel & $1.72 \AA /$ pixel \\
Wavelength range & $3570-5770 \AA$ & $3700-5269 \AA$ & $3570-5770 \AA$ & $3700-6151 \AA$ \\
Spectral resolution & $3.6 \AA$ (FWHM) & $3.5 \AA$ & $3.6 \AA$ & $6.56 \AA$ \\
Slit width & 2.1 arcsec & 2.0 arcsec & 2.1 arcsec & 2.0 arcsec \\
Spatial scale & 1.1 arcsec/pixel & 0.8 arcsec/pixel & 1.1 arc/pixel & 0.8 arcsec/pixel \\
\hline
\end{tabular}
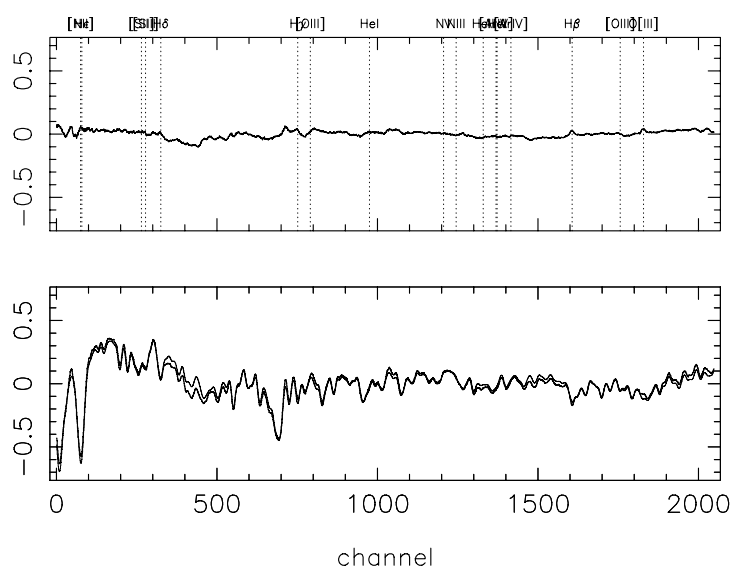

Fig. 2. Example of determination of kinematical parameters in the case of the galaxy NGC 3379. The upper panel shows the residuals of the optimal template fit, with the vertical dotted lines indicating the position of typical emission lines. In the lower panel, the thick and thin lines correspond to the galaxy and optimal template spectra, respectively.

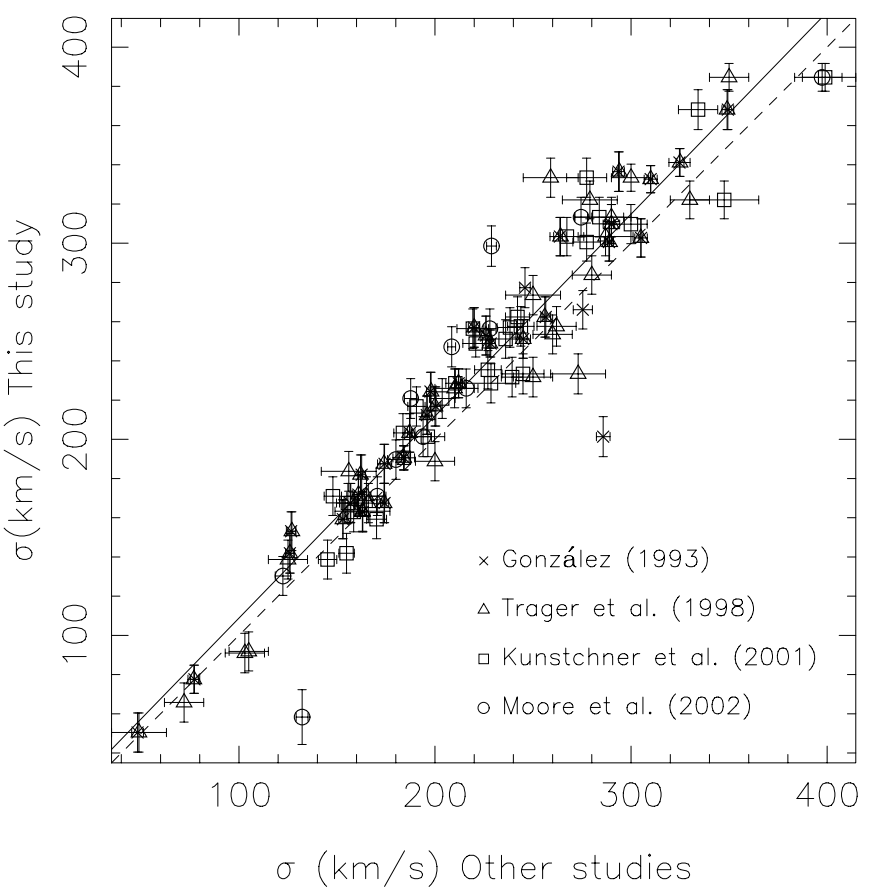

Fig. 3. Comparison of the derived velocity dispersions calculated in this work with the data compiled from the literature. Different symbols represent different datasets, as indicated in the inset. The 1:1 relation and the linear fit to the data are drawn with dashed and solid lines, respectively.
Table 3. Comparison of the velocity dispersions derived in this study and in previous works. The table columns are: Offset: mean offset between this study and the one in Col. 1; rms: dispersion among the mean value; Exp. rms: dispersion expected from the errors; $z$ : result of a $z$-test to contrast the hypothesis of a null offset (a value higher than 1.96 allows us to reject the hypothesis with a significance level lower than $0.05) ; N$ : number of galaxies in each comparison. Units are $\mathrm{km} \mathrm{s}^{-1}$.

\begin{tabular}{lccccc}
\hline \hline Study & Offset & rms & Exp. rms & $z$ & $N$ \\
\hline G93 & 12.5 & 19.5 & 9.6 & 3.1 & 33 \\
T98 & 13.7 & 15.1 & 10.7 & 4.8 & 52 \\
M02 & 17.4 & 21.2 & 11.0 & 1.7 & 12 \\
K01 & 12.9 & 15.8 & 11.9 & 3.5 & 31 \\
\hline
\end{tabular}

Table 4. Typical values of the different considered error sources. Phot.: photonic noise; Flux: flux calibration; Wave.: wavelength calibration; Dichr.: typical error in the subtraction of the dichroic pattern (this error is only added in the measurements of Run 2). Res.: residual errors, based on repeated observations of 10 galaxies.

\begin{tabular}{lccccl}
\hline \hline Index & Phot. & Flux & Wave. & $\begin{array}{c}\text { Dichr. } \\
\text { (Run 2) }\end{array}$ & Res. \\
\hline $\mathrm{D} 4000$ & 0.006 & 0.088 & 0.000 & 0.001 & $0.000 \AA$ \\
$\mathrm{H} \delta_{A}$ & 0.156 & 0.038 & 0.005 & 0.053 & $0.000 \AA$ \\
$\mathrm{H} \delta_{F}$ & 0.104 & 0.009 & 0.009 & 0.021 & $0.000 \AA$ \\
$\mathrm{CN}_{1}$ & 0.004 & 0.002 & 0.000 & 0.003 & $0.001 \mathrm{mag}$ \\
$\mathrm{CN}_{2}$ & 0.005 & 0.003 & 0.000 & 0.003 & $0.010 \mathrm{mag}$ \\
$\mathrm{Ca} 4227$ & 0.072 & 0.002 & 0.006 & 0.023 & $0.000 \AA$ \\
$\mathrm{G} 4300$ & 0.121 & 0.014 & 0.013 & 0.138 & $0.074 \AA$ \\
$\mathrm{H} \gamma_{A}$ & 0.143 & 0.033 & 0.002 & 0.121 & $0.302 \AA$ \\
$\mathrm{H} \gamma_{F}$ & 0.087 & 0.012 & 0.003 & 0.062 & $0.072 \AA$ \\
$\mathrm{Fe} 4383$ & 0.167 & 0.045 & 0.019 & 0.114 & $0.161 \AA$ \\
$\mathrm{Ca} 4455$ & 0.086 & 0.004 & 0.024 & 0.024 & $0.000 \AA$ \\
$\mathrm{Fe} 4531$ & 0.127 & 0.015 & 0.012 & 0.026 & $0.000 \AA$ \\
$\mathrm{C} 4668$ & 0.186 & 0.171 & 0.010 & 0.012 & $0.054 \AA$ \\
$\mathrm{H} \beta$ & 0.078 & 0.010 & 0.007 & 0.003 & $0.029 \AA$ \\
$\mathrm{Fe} 5015$ & 0.167 & 0.081 & 0.022 & 0.025 & $0.115 \AA$ \\
$\mathrm{Mgb}$ & 0.087 & 0.035 & 0.006 & 0.108 & $0.029 \AA$ \\
$\mathrm{Fe} 5270$ & & & & & $0.050 \AA$ \\
$\mathrm{Fe} 5335$ & & & & & $0.000 \AA$ \\
\hline
\end{tabular}

spectra. The errors were estimated from the uncertainties caused by photon noise, wavelength calibration, and flux calibration. We also added a residual error based on the comparison of galaxies observed in several runs and, in Run 2, the error due to the dichroic correction. Table 4 shows the typical values of the different sources of errors.

Table D.3 presents the fully corrected line-strength indices for our sample of galaxies. 
Table 5. Resolution at which Lick indices are measured.

\begin{tabular}{lrlr}
\hline \hline Index & $\begin{array}{r}\text { Resolution } \\
\left(\mathrm{km} \mathrm{s}^{-1}\right)\end{array}$ & Index & $\begin{array}{r}\text { Resolution } \\
\left(\mathrm{km} \mathrm{s}^{-1}\right)\end{array}$ \\
\hline $\mathrm{H} \delta_{A}$ & 325 & $\mathrm{Ca} 4455$ & 250 \\
$\mathrm{H} \delta_{F}$ & 325 & $\mathrm{Fe} 4531$ & 250 \\
$\mathrm{CN}_{1}$ & 325 & $\mathrm{C} 4668$ & 250 \\
$\mathrm{CN}_{2}$ & 325 & $\mathrm{H} \beta$ & 225 \\
$\mathrm{Ca} 4227$ & 300 & $\mathrm{Fe} 5015$ & 200 \\
$\mathrm{G} 4300$ & 300 & $\mathrm{Mgb}$ & 200 \\
$\mathrm{H} \gamma_{A}$ & 275 & $\mathrm{Fe} 5270$ & 200 \\
$\mathrm{H} \gamma_{F}$ & 275 & $\mathrm{Fe} 5335$ & 200 \\
$\mathrm{Fe} 4383$ & 250 & & \\
\hline
\end{tabular}

\subsection{Conversion to the Lick/IDS system}

The line-strength indices were transformed to the Lick system, taking into account three effects: (a) the differences in the spectral resolution between the Lick/IDS system and our setups, (b) the internal velocity broadening of the observed galaxies, and (c) small systematic offsets due to the continuum shape differences.

(a) To account for the differences in spectral resolution, we broadened the observed spectra with a Gaussian of wavelength-dependent width, following the prescriptions of Gorgas et al. (2006, in preparation). These authors estimated the resolution at which each particular index should be measured by broadening stars in common with the Lick library to several resolutions in steps of $25 \mathrm{~km} \mathrm{~s}^{-1}$. They then calculated an approximate resolution that, changing gently with wavelength, minimized the residuals. These values are given in Table 5. In general, the values calculated by these authors agree with the estimates given in Worthey \& Ottaviani (1997).

(b) In the second step, we corrected our indices for velocity dispersion broadening. We followed the standard procedure of determining correction curves from artificially broadened stellar spectra. However, instead of using individual stars, we broadened the different composite templates obtained in the determination of the kinematics parameters (Sect. 3). This was done because, in principle, one might expect a dependence of the correction factor on line-strength. The indices were then measured for each template and a correction factor of the form $C(\sigma)=I(0) / I(\sigma)$ (for the atomic indices) and $C(\sigma)=I(0)-I(\sigma)$ (for the molecular indices, $\mathrm{H} \delta_{A}$ and $\left.\mathrm{H} \delta_{F}\right)$ was determined for each galaxy, where $I(0)$ represents the index corrected from the broadening, and $I(\sigma)$ is the index measured in a spectrum broadened by $\sigma$. Although we derived different polynomials for each galaxy, the final correction factors were obtained by taking the mean of all the 98 templates in each $\sigma$-bin. This allowed us to quantify the errors due to this correction. Appendix $\mathrm{C}$ shows the dependence of the correction factor on $\sigma$ for all the measured indices and the derived uncertainties.

(c) The original Lick/IDS spectra were not flux-calibrated by means of a flux-standard stars but normalised to a quartziodide tungsten lamp. The resulting continuum shape cannot be reproduced and causes significant offsets for indices with a broad wavelength coverage. To calculate these offsets, we observed 20, 28, 9, and 30 different Lick stars in the first, second, third, and forth observing runs, respectively. By comparing the indices measured in our stars with those in the Lick/IDS database for the same objects, we derived mean offsets for all the indices in each observing run. However, the final offsets were obtained as an average of the offsets in all the runs, weighted by the number of observed stars. We do not apply different offsets to each run, as they, in principle, are expected to be the same (as our spectra are flux calibrated). Appendix A, available in electronic form at http: //www . edpsciences . org, summarises the comparison with the Lick/IDS stars. The last column of Table A.1 shows the final offsets applied to all the analysed indices.

Differences in the derived offsets between runs could appear due to systematic errors in the flux calibration, but these possible errors affect stars and galaxies differently, and they are taken into account in the comparison between galaxies observed in different runs. Systematic differences in the offsets between runs could also appear if the Lick resolution was not matched perfectly in all the runs. As the resolution in Run 4 is very different from that of the other runs, we have compared, in Appendix B, the indices measured from stars observed in both Run 4 and other runs. We have not found any significant difference. In the special case of the D4000, we compared the stars in common with the library of Gorgas et al. (1999). The estimated offset for this index is zero.

Finally, we calculated a residual error for each index based on repeated observations of galaxies in different runs. We have a total of 11 repeated observations of 10 different galaxies. The residual error was computed by comparing the rms dispersion with the estimated errors from known sources. The final residual errors are shown in the last column of Table 4.

\subsection{Emission correction}

Elliptical galaxies contain much less dust and ionised gas than spiral galaxies and were regarded as dust- and gas-free objects for a long time. However, surveys of large samples of early-type galaxies (Caldwel 1984; Phillips et al. 1986; Goudfrooij et al. 1994) have revealed that 50-60 per cent of these galaxies show weak optical emission lines. Some line-strength indices are affected by these emission lines, in particular $\mathrm{H} \beta, \mathrm{Fe} 5015$, and $\mathrm{Mgb}$. The effect of the emission on $\mathrm{H} \beta$ is particularly important because our estimation of ages (Paper II) relies on its strength. The emission, when present, tends to fill the line, lowering the value of the index and, hence, increasing the derived age. To correct the $\mathrm{H} \beta$ index from this effect, we used the calibration of G93, who showed that $\mathrm{H} \beta$ is correlated with [O III] such that $E W\left(\mathrm{H} \beta_{\mathrm{em}}\right) / E W([\mathrm{O} \mathrm{III}] \lambda$ 5007) 0.7. Trager et al. (2000a; hereafter T00a) carefully studied the accuracy of this correction, finding a better value of 0.6 instead of 0.7 . Whilst there is evidence that this correction factor is uncertain for individual galaxies (Mehlert et al. 2000), it is good enough in a statistical sense. In any case, we have repeated the analysis eliminating all the galaxies with [O III] $\lambda 5007>0.4 \AA$ and none of the results presented in this work suffer variation. To determine the [O III] emission, we subtracted an emission-free template spectrum from the galaxy spectrum and measured the residual equivalent width. The zero-emission template was the one calculated during the determination of the kinematics parameters, as described in Sect. 3. An example of the process is shown in Fig. 4. The emission lines [O III] $\lambda 5007$ and [N II] $\lambda 5199$, which affect the measurements of Fe5015 and $\mathrm{Mgb}$, were replaced with the optimal template in these regions. A total of 37 galaxies (38\% of our sample) were found to have evidence of [O III] $\lambda 5007$ emission $(E W([\mathrm{O}$ III $])>0.2 \AA)$. The [O III] equivalent widths measured in the central spectra are presented in the last column of Table D.3. 


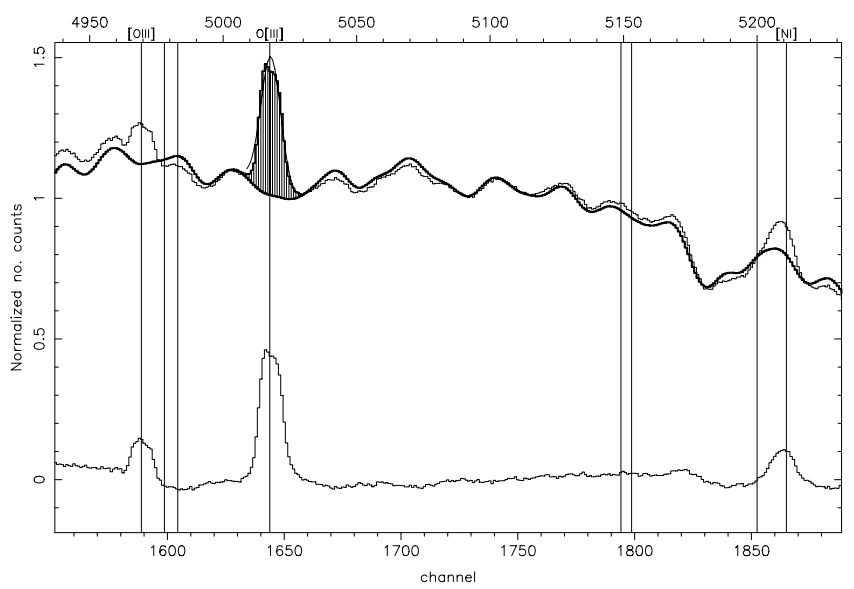

Fig. 4. Illustrative example of the procedure followed to measure emission lines. The upper part of the figure shows the galaxy (NGC 4278; thin line) and the template spectrum corrected for the kinematics parameters (thick line). The difference between both spectra (observed - template) is shown at the bottom of the figure. The equivalent width of the emission line [O III] $\lambda 5007$ is highlighted in the shaded area. The vertical lines indicate the expected location of the [O III] $] \lambda 5007,[\mathrm{~N}$ II $] \lambda 5199$, and several sky lines.

\subsection{Comparison with other authors}

To verify that our measurements have been properly transformed into the Lick system and that the errors have been properly estimated, we have compared our line strengths with data from 6 different works with galaxies in common: (i) G93, (ii) T98, (iii) Mehlert et al. (2000, hereafter M00), (iv) Kuntschner et al. (2001, hereafter K01), (v) Moore et al. (2002, hereafter M02), and (vi) Denicoló et al. (2005a, hereafter D05). Figure 5 shows this comparison and Table 6 summarises the mean offsets between different studies and the root mean square dispersions.

In general, our measurements agree with other studies fairly well, although we find significant offsets in the values of some indices. In particular, Mgb and C4668 seem to be depressed in our sample compared with the other studies. We will address throughout the paper the possible variation in our conclusions should an offset of -0.2 and -0.848 in Mgb and C4668 (corresponding to the mean difference in the comparison with other authors) be assumed.

We can obtain a good estimation of the quality of our errors comparing the dispersion along the 1:1 relation with the one expected from the errors. The last column of Table 6 shows the probability that the real scatter is larger than the one expected from the errors by chance. As can be seen, in most of the cases the observed scatter agrees with the one predicted from the errors, which indicates that the errors have been correctly estimated. However, this does not happen in the comparison with D05. As this is the only study in which the real scatter is significatively larger than the one expected by the errors, we think that these authors have underestimated the errors in their measurements.

Furthermore, we have used the galaxies in common with other studies to double check the possibility of systematic differences between different observing runs confirming the absence of these differences. This comparison can be found in Appendix D, available in electronic form at http: //www . edpsciences.org.

\section{The index-velocity dispersion relations}

The relations between the central velocity dispersion and the strength of the integrated stellar $\mathrm{Mg}$ and $\mathrm{MgH}$ features around $5100 \AA$ have been studied in numerous works (e.g., Terlevich et al. 1981; Gorgas et al. 1990; Guzmán et al. 1992; Bender et al. 1993; Jørgensen et al. 1996; Bender et al. 1998; Bernardi et al. 1998; Colless et al. 1999; Jørgensen 1999; Concannon et al. 2000; Kuntschner 2000; Poggianti et al. 2001a; Proctor \& Sansom 2002; Worthey \& Collobert 2003; Mehlert et al. 2003; Thomas et al. 2004). Although, classically, the $\mathrm{Mg}_{2}-\sigma$ relation has been interpreted as the interplay between mass and metallicity (Forbes et al. 1998; Kobayashi \& Arimoto 1999; Terlevich et al. 1999), nowadays there is still much debate as to whether this relation reflects trends in stellar ages, in metallicities, or in the relative abundance of different heavy elements (e.g., T98; Jørgensen 1999; Trager et al. 2000b; K01; Poggianti et al. 2001a; Mehlert et al. 2003; Caldwell et al. 2003; Thomas et al. 2005). The problem is due to the well-known agemetallicity degeneracy, which makes very difficult to separate both effects with the current stellar population models. However, the use of a larger number of indices can help us to disentangle this degeneracy, thanks to the different sensitivity of each index to variations of these parameters.

So far, most studies have concentrated on the relation of the $\mathrm{Mg}$ indices with the velocity dispersion. In this section, we show the relation of 18 Lick indices with $\sigma$. Some of the relations have already been presented by Sánchez-Blázquez et al. (2003), but they are shown here again for completeness. Most of them, however, are new. We aim to answer two questions:

1. Which parameter(s) is(are) responsible for the correlation between the indices and velocity dispersion?

2. Which parameter(s) is(are) responsible for the intrinsic scatter in these relations?

Following Colles et al. (1999), we will express the atomic indices in magnitudes. These indices will be denoted by the name followed by a prime sign, and are obtained as

$I^{\prime}=-2.5 \log \left(1-\frac{I}{\Delta \lambda}\right)$

where $I$ is the classic index measured in $\AA$ (atomic index), and $\Delta \lambda$ is the width of the index central bandpass. The D4000 was transformed to magnitudes as

$\mathrm{D} 4000^{\prime}=-2.5 \log (\mathrm{D} 4000)$.

Figure 6 shows the index $-\sigma$ relations for all the indices measured in this work. The fits were performed using an ordinary least-squares method, minimising the residuals in both the $x$-and $y$-directions. The method initially performs a typical unweighted ordinary least-squares regression of $\mathrm{Y}$ on $\mathrm{X}$, and the coefficients from the first fit are then employed to derive (numerically, with a downhill method) the straight line data fit with errors in both coordinates. The best linear relation $\left(I^{\prime}=a+b \log \sigma\right)$ and the scatter are summarised in Table 7a for the two different subsamples considered in this paper, HDEGs and LDEGs. From the LDEGs sample, two outliers were eliminated, NGC 4742 and IC 767, and from the HDEGs sample, NGC 6166 was also eliminated. The probability that the parameters $\left(I^{\prime}\right.$ and $\left.\sigma\right)$ are not correlated $(\alpha)$ was derived from a (non-parametric) Spearman rank-order test and is also shown in Table 7a. However, the Spearman test does not take into account the errors of the individual measurements. For this reason, we also carried out a $t$-test 

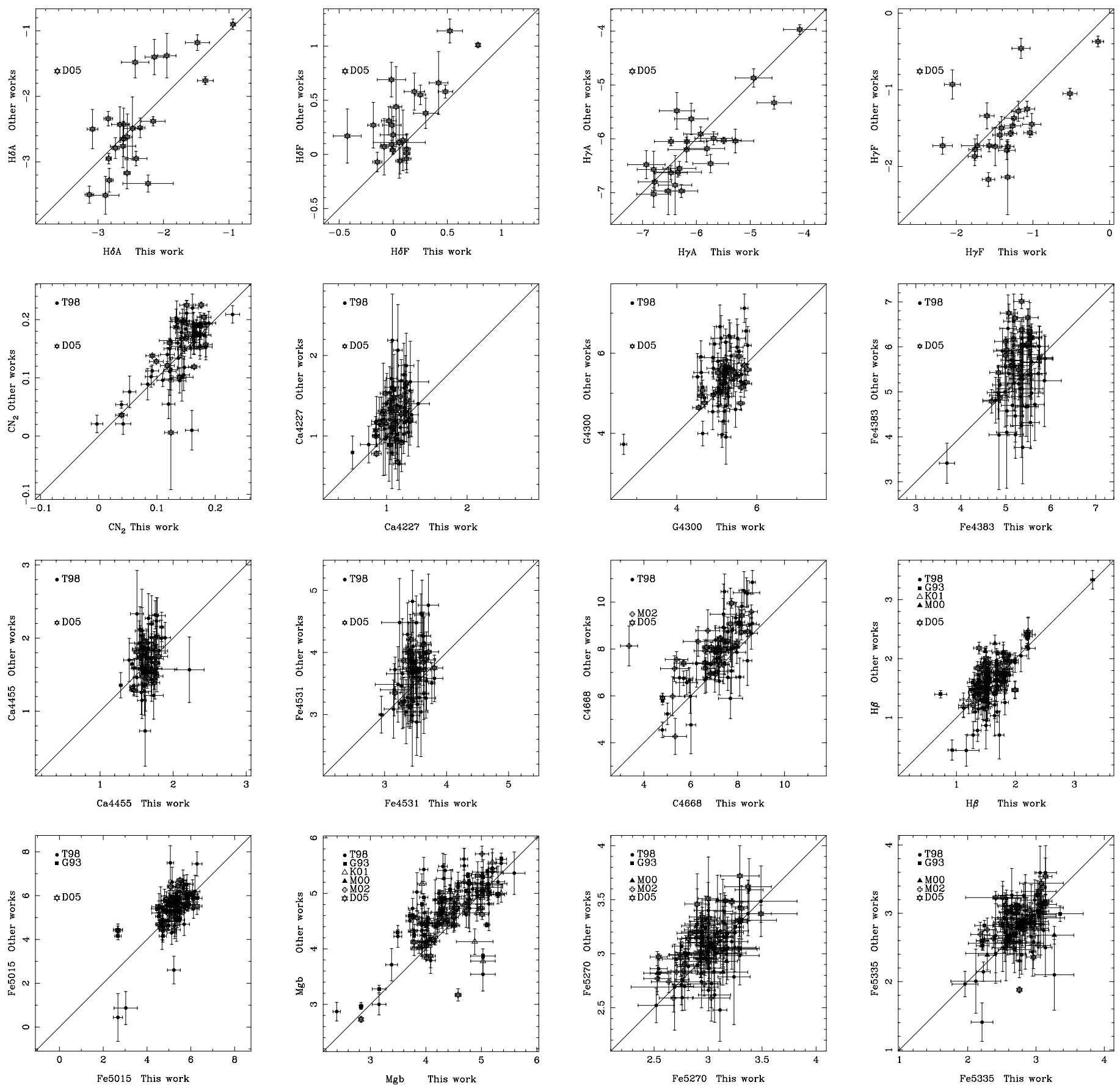

Fig. 5. Comparison between the Lick indices measured in this study with those in other works for the galaxies in common. Different symbols represent distinct references, as indicated in the insets.

to check the hypothesis $b=0$, where $b$ represents the slope of the fit. Values of $t$ larger than 1.96 indicate that the hypothesis can be rejected with a significance level lower than $\alpha=0.05$. To obtain the errors in the slope, 1000 Monte Carlo simulations were performed, perturbing the points randomly in both the $x$ and $y$-directions with a Gaussian distributions of width given by their errors.

\subsection{The slope of the relations}

To study which parameters drive the relation of the indices with $\sigma$, we have parameterised the models of V06 as a function of age and metallicity. The relations are shown in Table $7 \mathrm{~b}$.
In estimating these expressions, we have restricted the age to the interval $4.7-17.78 \mathrm{Gyr}$, and the metallicity range to $-0.68<$ $[\mathrm{M} / \mathrm{H}]<+0.2$, which is the range covered by the galaxies in our sample. To show that, we have plotted, in Fig. 7, an index-index diagram comparing the $[\mathrm{MgFe}]^{\prime}$ (Thomas et al. 2003, TMB03 hereafter) and the $\mathrm{H} \beta$ indices, including the LDEGs (open symbols) and HDEG (filled symbols). We have also over-plotted the grid of models by V06. The caption of the figure indicates the corresponding values of age and metallicity for each model. The two last columns of Table 7b give, for the LDEGs and HDEGs, respectively, the required dependence of metallicity on $(\log \sigma)$ if the observed slope of the particular index in the index- $\log \sigma$ diagram were to be driven entirely by a metallicity dependence 
Table 6. Comparison of line strengths measured in this and other studies. Ref.: reference of the comparison work (see description in the text); $N$ : number of galaxies in common; $\Delta I$ : calculated offset between both studies (this work minus other study); $\sigma$ : rms dispersion; $\sigma_{\text {exp }}$ : expected rms from the errors; $t: t$-parameter of the comparison of means; $\alpha$ : probability that $\sigma>\sigma_{\text {exp }}$ by chance.

\begin{tabular}{|c|c|c|c|c|c|c|c|}
\hline$\overline{\overline{\text { Index }}}$ & $\overline{\text { Ref. }}$ & $\overline{\bar{N}}$ & $\overline{\overline{\Delta I I}}$ & $\bar{\sigma}$ & $\overline{\sigma_{\exp }}$ & $\bar{t}$ & $\bar{\alpha}$ \\
\hline$\overline{\mathrm{H} \delta_{A}}$ & D05 & 24 & +0.305 & 0.442 & 0.569 & 2.76 & 0.006 \\
\hline $\mathrm{H} \delta_{F}$ & D05 & 24 & -0.036 & 0.197 & 0.450 & 0.87 & 0.197 \\
\hline $\mathrm{H} \gamma_{A}$ & D05 & 24 & +0.550 & 0.428 & 0.555 & 3.82 & 0.001 \\
\hline $\mathrm{H} \gamma_{F}$ & D05 & 24 & +0.349 & 0.359 & 0.463 & 3.38 & 0.001 \\
\hline $\mathrm{CN}_{2}$ & T98 & 53 & -0.010 & 0.033 & 0.195 & 2.22 & 0.015 \\
\hline $\mathrm{CN}_{2}$ & D05 & 24 & +0.125 & 0.034 & 0.094 & 4.64 & 0.001 \\
\hline $\mathrm{Ca} 4427$ & T98 & 53 & -0.181 & 0.287 & 0.755 & 3.88 & 0.001 \\
\hline $\mathrm{Ca} 4227$ & D05 & 24 & -0.070 & 0.170 & 0.341 & 1.85 & 0.039 \\
\hline G4300 & T98 & 53 & -0.272 & 0.640 & 0.807 & 2.84 & 0.003 \\
\hline G4300 & D05 & 24 & +0.061 & 0.310 & 0.506 & 0.94 & 0.179 \\
\hline $\mathrm{Fe} 4383$ & T98 & 53 & -0.051 & 0.561 & 1.081 & 0.65 & 0.259 \\
\hline $\mathrm{Fe} 4383$ & D05 & 24 & -0.639 & 0.463 & 0.615 & 3.91 & 0.001 \\
\hline $\mathrm{Ca} 4455$ & T98 & 53 & -0.150 & 0.304 & 0.700 & 3.21 & 0.001 \\
\hline $\mathrm{Ca} 4455$ & D05 & 24 & +0.170 & 0.210 & 0.419 & 3.05 & 0.003 \\
\hline $\mathrm{Fe} 4531$ & T98 & 53 & -0.205 & 0.422 & 0.887 & 3.18 & 0.001 \\
\hline $\mathrm{Fe} 4531$ & D05 & 24 & -0.175 & 0.217 & 0.411 & 3.05 & 0.003 \\
\hline C4668 & T98 & 53 & -0.667 & 0.821 & 1.129 & 4.57 & 0.001 \\
\hline C4668 & M02 & 12 & -1.194 & 1.332 & 1.143 & 2.19 & 0.027 \\
\hline C4668 & D05 & 24 & -0.684 & 0.541 & 0.671 & 3.79 & 0.001 \\
\hline $\mathrm{H} \beta$ & T98 & 53 & +0.121 & 0.256 & 0.621 & 3.10 & 0.001 \\
\hline $\mathrm{H} \beta$ & G93 & 33 & -0.057 & 0.150 & 0.333 & 2.16 & 0.019 \\
\hline $\mathrm{H} \beta$ & K01 & 32 & +0.098 & 0.187 & 0.565 & 2.62 & 0.007 \\
\hline $\mathrm{H} \beta$ & M00 & 6 & -0.428 & 0.316 & 0.418 & 1.86 & 0.068 \\
\hline $\mathrm{H} \beta$ & D05 & 24 & +0.004 & 0.491 & 0.357 & 0.04 & 0.484 \\
\hline Fe5015 & T98 & 53 & -0.187 & 1.030 & 0.739 & 1.79 & 0.040 \\
\hline Fe5015 & G93 & 33 & -0.451 & 0.465 & 0.612 & 2.22 & 0.017 \\
\hline Fe5015 & D05 & 24 & -0.208 & 0.653 & 0.574 & 1.48 & 0.076 \\
\hline Mgb & T98 & 53 & -0.376 & 0.478 & 0.717 & 4.49 & 0.001 \\
\hline Mgb & G93 & 33 & -0.535 & 0.601 & 0.386 & 2.20 & 0.018 \\
\hline Mgb & K01 & 32 & -0.047 & 0.431 & 0.615 & 0.61 & 0.273 \\
\hline Mgb & M00 & 6 & -0.158 & 0.220 & 0.491 & 1.39 & 0.118 \\
\hline $\mathrm{Mgb}$ & M02 & 12 & -0.160 & 0.125 & 0.518 & 2.66 & 0.012 \\
\hline Mgb & D05 & 24 & +0.061 & 0.443 & 0.467 & 0.67 & 0.255 \\
\hline $\mathrm{Fe} 5270$ & T98 & 53 & +0.009 & 0.340 & 0.761 & 0.19 & 0.425 \\
\hline $\mathrm{Fe} 5270$ & G93 & 33 & +0.004 & 0.027 & 0.322 & 0.61 & 0.273 \\
\hline $\mathrm{Fe} 5270$ & M00 & 6 & +0.212 & 0.153 & 0.480 & 1.87 & 0.067 \\
\hline $\mathrm{Fe} 5270$ & M02 & 12 & +0.118 & 0.213 & 0.495 & 1.66 & 0.064 \\
\hline $\mathrm{Fe} 5270$ & D05 & 24 & -0.057 & 0.181 & 0.399 & 1.46 & 0.079 \\
\hline Fe5335 & T98 & 53 & +0.009 & 0.340 & 0.761 & 0.19 & 0.425 \\
\hline Fe5335 & G93 & 33 & -0.009 & 0.018 & 0.340 & 1.79 & 0.042 \\
\hline Fe5335 & M00 & 6 & +0.111 & 0.398 & 0.514 & 0.65 & 0.275 \\
\hline Fe5335 & M02 & 12 & +0.035 & 0.373 & 0.598 & 0.32 & 0.378 \\
\hline Fe5335 & D05 & 24 & +0.040 & 0.431 & 0.441 & 0.45 & 0.328 \\
\hline$<\mathrm{Fe}>$ & K01 & 31 & -0.016 & 0.183 & 0.159 & 0.65 & 0.110 \\
\hline
\end{tabular}

on $\log \sigma$. This is also illustrated in Fig. 8 for both subsamples, LDEGs and HDEGs. As can be seen in Table 7b, the metallicity dependence required to explain the slope of the $\mathrm{CN}_{2}, \mathrm{C} 4668$, and $\mathrm{Mgb}$ indices with $\sigma$ is much larger than that required to explain the slope of the other indices. Therefore, the metallicity cannot be the only parameter varying with the velocity dispersion. It does not seem probable, either, that a unique combination of age and metallicity is responsible for the differences in the slope between indices, since $\mathrm{CN}_{2}, \mathrm{C} 4668$, and $\mathrm{Mgb}$ are not especially sensitive to age variations (e.g., Worthey 1994). A dependence of age on velocity dispersion in the galaxies would produce steeper slopes in other indices, such as G4300 or Fe4531.

The most plausible explanation is the existence of systematic changes in the chemical abundance ratios along the $\sigma$ sequence.
That is, not all the elements are changing in lockstep with the velocity dispersion. As different indices have different sensitivities to changes in the chemical composition, a variation of the latter with $\sigma$ would produce differences in the slope of the relation. Although we do not have the tools to derive detailed chemical abundances in early-type galaxies, we know that the index $\mathrm{CN}_{2}$ is especially sensitive to variations of $\mathrm{C}$ and $\mathrm{N}, \mathrm{C} 4668$ to variations in the $\mathrm{C}$ abundance, while the $\mathrm{Mgb}$ index increases with the $\mathrm{Mg}$ abundance, and it has an inverse dependence with the $\mathrm{C}$ abundance (Tripicco \& Bell 1995, TB95 hereafter; Korn et al. 2005). Therefore, the explanation of the differences in the slopes of these indices with respect to the others would require, in principle, an increase of the $[\mathrm{Mg} / \mathrm{Fe}]$ (already noted by a number of authors, e.g., Worthey et al. 1992; Greggio 1997; Jørgensen 1999; Kuntschner 2000; Trager et al. 2000b; Thomas et al. 2002; M03; Thomas et al. 2005), [C/Fe] and [N/Fe] (see Worthey 1998) ratios with $\sigma$.

However, there are other indices that also depend strongly on these elements and, thus, before reaching the above conclusion, we should study whether the variations in the relative abundance of these elements with $\sigma$ are compatible with the slopes obtained for the rest of the indices.

Using a similar procedure to T00a, we have used the response functions of TB95 to calculate the variation of the Lick indices to changes in the abundances of different elements.

Instead of changing all the elements separately, we assume that some elements are linked nucleosynthetically, so we vary them in lockstep. Following T00a, we separate the elements into two different groups: (G1) Na, Si, Ca, O, and $\mathrm{Ti}^{1}$; (G2) Fe and $\mathrm{Cr}$. In addition, we allow the abundance of $\mathrm{C}, \mathrm{N}$, and $\mathrm{Mg}$ to vary independently. TB95 response functions are for enhancement values corresponding to $[\mathrm{X} / \mathrm{H}]=+0.3 \mathrm{dex}$. The fractional response of an index $I$ to other arbitrary values is (see T00a)

$$
\frac{\Delta I}{I_{0}}=\left\{\prod_{i}\left[1+R_{0.3}\left(\mathrm{X}_{i}\right)\right]^{\left(\left[\mathrm{X}_{i} / \mathrm{H}\right] / 0.3\right)}\right\}-1,
$$

where $R_{0.3}\left(\mathrm{X}_{i}\right)$ is the TB95 response function for the $i$ th element at $\left[\mathrm{X}_{i} / \mathrm{H}\right]=+0.3$ dex.

To compute the final index variations we have assumed the following composition: $53 \%$ of cool giants, $44 \%$ of turn off stars, and $3 \%$ of cool dwarfs stars, as in T00a.

We calculated the changes in the indices that are induced by changing the abundance ratio patterns according to the following four different prescriptions:

- Model (i): $\mathrm{G} 1[\mathrm{X} / \mathrm{H}]=+0.5$ (including $\mathrm{C}, \mathrm{N}$ and $\mathrm{Mg}$ ) and $\mathrm{G} 2[\mathrm{X} / \mathrm{H}]=+0.3$.

- Model (ii): $\mathrm{G} 1[\mathrm{X} / \mathrm{H}]=+0.5$ (including $\mathrm{C}, \mathrm{N}$ and $\mathrm{Mg}$ ) and $\mathrm{G} 2[\mathrm{X} / \mathrm{H}]=+0.0$.

- Model (iii): $\mathrm{G} 1[\mathrm{X} / \mathrm{H}]=+0.5$ (including $\mathrm{N}$ and $\mathrm{Mg}$ ) and $\mathrm{G} 2$ $[\mathrm{X} / \mathrm{H}]=+0.3$ (including $\mathrm{C}$ ).

- Model (iv): $\mathrm{G} 1 \quad[\mathrm{X} / \mathrm{H}]=+0.5, \quad \mathrm{G} 2 \quad[\mathrm{X} / \mathrm{H}]=+0.3$, $[\mathrm{C} / \mathrm{H}]=+0.43,[\mathrm{~N} / \mathrm{H}]=+0.63$, and $[\mathrm{Mg} / \mathrm{H}]=+1.2$.

The first three models are simple variations of the different proposed groups and are shown here to show the influence that the variation of different groups of elements have over the slopes of the indices with $\sigma$. In the fourth model, on the other hand, we have fixed the values for G1 and G2 as in model (iii), which is

\footnotetext{
1 Although the nucleosynthesis theory of Ti is not well understood, this element appears to be enhanced in most bulge stars by $\sim 0.3 \mathrm{dex}$ (e.g., see McWilliam \& Rich 2004).
} 

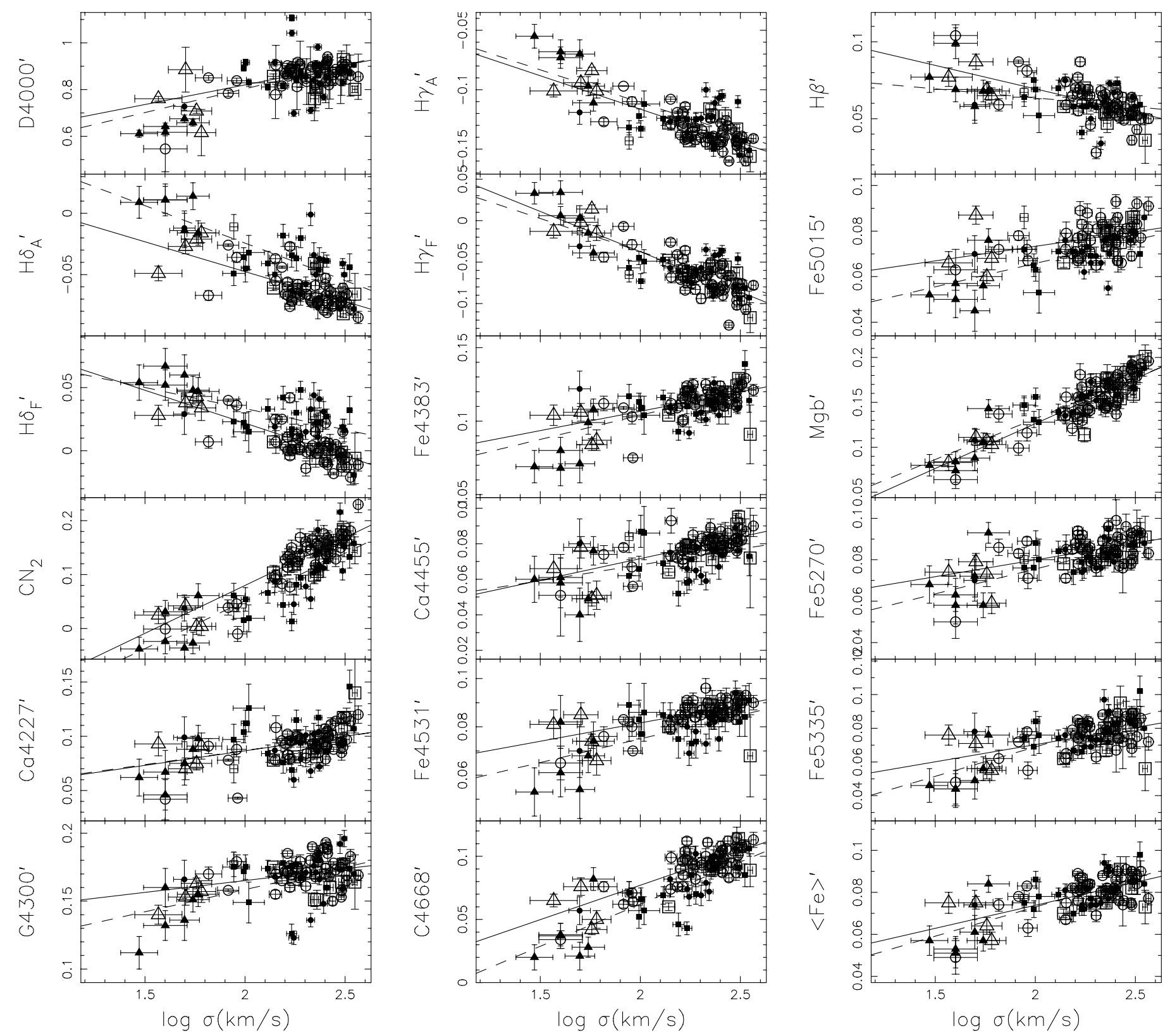

Fig. 6. Line-strength indices plotted against $\log \sigma$. Open symbols show the galaxies in low-density environments and filled symbols, galaxies in the Coma cluster. The different shapes represent distinct morphological types (triangles: dwarfs; squares: S0; circles: ellipticals). All indices are measured in magnitudes following the conversion of Eq. (1). Solid and dashed lines represent error-weighted least-squares linear fits to LDEGs and HDEGs, respectively.

the one with lower rms of the first three models, and we have fitted the values of $\mathrm{C}, \mathrm{N}$, and $\mathrm{Mg}$ to reproduce the observed slopes in $\mathrm{CN}_{2}, \mathrm{C} 4668$, and $\mathrm{Mgb}$.

Figure 9 shows the comparison of the slopes with the variation of the indices in the different proposed models. The rms of the dispersion and the mean differences in the indices modeled by TB95 are summarised in Table 8 . As it is apparent, the model that best reproduces the observed slopes is model (iv), in which the $\mathrm{Mg}$ and the $\mathrm{N}$ change more than the other $\alpha$-elements, while the $\mathrm{C}$ changes more than the Fe-peak elements, but less than the $\alpha$-elements. However, this variation of $\mathrm{C}$ gives slopes in the G4300 index (also very C sensitive) steeper than observed. The observations of this index do not fit well with the stellar atmosphere model of the coolest giant star of TB95. Therefore, the differences may also be due to a problem in the modeling.
On the other hand, there is a possibility that the C4668 index is not as sensitive to variations in the $\mathrm{C}$ abundance as predicted by TB95 (Worthey 2004). Therefore, we cannot make firm conclusions about the variation of the $[\mathrm{C} / \mathrm{Fe}]$ abundance with the velocity dispersion of the galaxies. The reported values for the different models are only illustrative. We do not pretend to quantify the real variations in the chemical abundance ratios with the velocity dispersion, but only to obtain relative variation of some elements with respect to the others. To explain the differences in the slope of the relations of $\mathrm{Mgb}$ and $\langle\mathrm{Fe}\rangle$ with $\sigma$, some authors have already proposed the existence of an increase in the $\mathrm{Mg} / \mathrm{Fe}$ ratio with the velocity dispersion (K01; Proctor \& Sansom 2002; Mehlert et al. 2003; but, for an opposing alternative, see Proctor et al. 2004). In this work, we propose that, apart from the variation in this ratio, there is also a variation in the $[\mathrm{N} / \mathrm{Fe}]$ ratio with 
Table 7. a) Parameters of the linear fits $I^{\prime}=a+b \log \sigma$. First column: $y$-intersect of the fit and its error; second column: slope, and corresponding error, of the linear fit; $\alpha$ : probability, given by a non-parametric Spearman test, that the slope of the fit is significatively different from zero by chance; $N$ : number of galaxies used to make the fit; $t: t$ parameter to test the hypothesis $b=0$ (a $t$ value higher than 1.9 indicates that the slope is significant different from zero with a significance level lower than 0.05$) ; \sigma_{\text {std }}:$ standard deviation about the fit; $\sigma_{\text {exp }}$ : standard deviation expected from the errors; $\sigma_{\text {res }}$ : standard deviation not explained from the errors.

\begin{tabular}{|c|c|c|c|c|c|c|c|c|}
\hline \multicolumn{9}{|c|}{ LDEGs } \\
\hline Index & $a \pm \sigma(a)$ & $\overline{b \pm \sigma(b)}$ & $\bar{\alpha}$ & $N$ & $\bar{t}$ & $\bar{\sigma}_{\text {std }}$ & $\sigma_{\text {exp }}$ & $\sigma_{\text {res }}$ \\
\hline$\overline{\mathrm{D} 4000^{\prime}}$ & $0.484 \pm 0.016$ & $0.1679 \pm 0.007$ & 0.005 & 59 & 23.98 & 0.037 & 0.012 & 0.035 \\
\hline $\mathrm{H} \delta_{A}^{\prime}$ & $0.050 \pm 0.023$ & $-0.049 \pm 0.004$ & $9.22 \mathrm{E}-07$ & 59 & 4.83 & 0.032 & 0.002 & 0.032 \\
\hline $\mathrm{H} \delta_{F}^{\prime}$ & $0.126 \pm 0.016$ & $-0.052 \pm 0.007$ & $1.72 \mathrm{E}-09$ & 59 & 7.66 & 0.038 & 0.002 & 0.038 \\
\hline $\mathrm{CN}_{2}$ & $-0.276 \pm 0.035$ & $0.178 \pm 0.007$ & $3.08 \mathrm{E}-17$ & 59 & 12.96 & 0.023 & 0.006 & 0.023 \\
\hline $\mathrm{Ca} 4227^{\prime}$ & $0.033 \pm 0.011$ & $0.027 \pm 0.005$ & $5.93 \mathrm{E}-06$ & 59 & 2.20 & 0.008 & 0.004 & 0.007 \\
\hline G4300' & $0.130 \pm 0.008$ & $0.017 \pm 0.004$ & 0.054 & 59 & 1.96 & 0.017 & 0.003 & 0.017 \\
\hline $\mathrm{H} \gamma_{A}^{\prime}$ & $-0.004 \pm 0.010$ & $-0.056 \pm 0.004$ & 4.16E-09 & 59 & 6.52 & 0.026 & 0.002 & 0.026 \\
\hline $\mathrm{H} \gamma_{F}^{\prime}$ & $0.157 \pm 0.013$ & $-0.097 \pm 0.006$ & $9.65 \mathrm{E}-11$ & 59 & 9.85 & 0.025 & 0.004 & 0.025 \\
\hline Fe4383' & $0.054 \pm 0.007$ & $0.026 \pm 0.003$ & 2.97E-08 & 59 & 3.20 & 0.009 & 0.002 & 0.009 \\
\hline $\mathrm{Ca} 4455^{\prime}$ & $0.023 \pm 0.008$ & $0.024 \pm 0.003$ & $3.61 \mathrm{E}-07$ & 59 & 2.51 & 0.004 & 0.003 & 0.002 \\
\hline Fe4531' & $0.051 \pm 0.007$ & $0.015 \pm 0.003$ & $2.53 \mathrm{E}-07$ & 59 & 3.18 & 0.003 & 0.002 & 0.003 \\
\hline C4668' & $-0.032 \pm 0.001$ & $0.054 \pm 0.004$ & 2.23E-09 & 59 & 7.34 & 0.009 & 0.003 & 0.009 \\
\hline $\mathrm{H} \beta$, & $0.130 \pm 0.014$ & $-0.030 \pm 0.006$ & 1.37E-09 & 59 & 5.25 & 0.012 & 0.002 & 0.012 \\
\hline Fe5015' & $0.048 \pm 0.005$ & $0.013 \pm 0.002$ & $1.0 \mathrm{E}-04$ & 59 & 2.30 & 0.004 & 0.002 & 0.004 \\
\hline $\mathrm{Mgb}^{\prime}$ & $-0.073 \pm 0.012$ & $0.100 \pm 0.005$ & $1.22 \mathrm{E}-16$ & 59 & 10.78 & 0.011 & 0.002 & 0.011 \\
\hline $\mathrm{Fe} 5270^{\prime}$ & $0.047 \pm 0.010$ & $0.016 \pm 0.004$ & 0.001 & 59 & 3.60 & 0.004 & 0.002 & 0.004 \\
\hline Fe5335' & $0.029 \pm 0.013$ & $0.020 \pm 0.006$ & 0.030 & 59 & 3.52 & 0.003 & 0.003 & 0.000 \\
\hline
\end{tabular}

\begin{tabular}{|c|c|c|c|c|c|c|c|c|}
\hline \multicolumn{9}{|c|}{$\overline{\mathrm{HDEGs}}$} \\
\hline Index & $a \pm \sigma(a)$ & $b \pm \sigma(b)$ & $\alpha$ & $N$ & $t$ & $\sigma_{\text {std }}$ & $\sigma_{\exp }$ & $\sigma_{\text {res }}$ \\
\hline$\overline{\mathrm{D} 4000^{\prime}}$ & $0.397 \pm 0.019$ & $0.203 \pm 0.008$ & 0.071 & 36 & 23.87 & 0.098 & 0.014 & 0.097 \\
\hline $\mathrm{H} \delta_{A}^{\prime}$ & $0.098 \pm 0.018$ & $-0.061 \pm 0.009$ & $1.00 \mathrm{E}-04$ & 36 & 7.03 & 0.018 & 0.008 & 0.017 \\
\hline $\mathrm{H} \delta_{F}^{\prime}$ & $0.100 \pm 0.0205$ & $-0.033 \pm 0.009$ & 0.010 & 36 & 3.59 & 0.015 & 0.008 & 0.013 \\
\hline $\mathrm{CN}_{2}$ & $-0.303 \pm 0.043$ & $0.177 \pm 0.020$ & $1.44 \mathrm{E}-10$ & 36 & 8.81 & 0.034 & 0.013 & 0.031 \\
\hline $\mathrm{Ca} 4227^{\prime}$ & $0.035 \pm 0.023$ & $026 \pm 0.010$ & 0.125 & 36 & 2.51 & 0.011 & 08 & 0.007 \\
\hline G4300' & 1 & 0 & 0. & 36 & 3.41 & 0. & 06 & 0.014 \\
\hline $\mathrm{H} \gamma_{A}^{\prime}$ & -0.0 & $-0.055 \pm$ & 0.020 & 36 & 4.70 & 0.016 & 06 & 0.015 \\
\hline $\mathrm{H} \gamma_{F}^{\prime}$ & $0.122 \pm$ & $-0.080 \pm 0.013$ & $3.00 \mathrm{E}-04$ & 36 & 5.95 & 0.017 & 0.070 & 0.016 \\
\hline $\mathrm{Fe} 4383^{\prime}$ & $0.038 \pm 0.023$ & $0.033 \pm 0.010$ & 0.006 & 36 & 3.29 & 0.007 & 0.005 & 0.005 \\
\hline $\mathrm{Ca} 4455^{\prime}$ & $0.032 \pm 0.014$ & $0.018 \pm 0.006$ & 0.011 & 36 & 2.87 & 0.006 & 0.005 & 0.002 \\
\hline $\mathrm{Fe} 4531^{\prime}$ & $0.036 \pm 0.0120$ & $0.020 \pm 0.005$ & $5.0 \mathrm{E}-04$ & 36 & 3.70 & 0.006 & 0.005 & 0.0027 \\
\hline $\mathrm{Fe} 4668^{\prime}$ & $-0.071 \pm 0.018$ & $0.067 \pm 0.008$ & $2.19 \mathrm{E}-09$ & 36 & 8.25 & 0.012 & 0.006 & 0.010 \\
\hline $\mathrm{H} \beta^{\prime}$ & $0.087 \pm 0.009$ & $-0.012 \pm 0.004$ & 0.189 & 36 & 2.85 & 0.009 & 0.004 & 0.008 \\
\hline Fe5015' & $0.025 \pm 0.011$ & $0.021 \pm 0.005$ & $1.46 \mathrm{E}-05$ & 36 & 4.38 & 0.004 & 0.003 & 0.002 \\
\hline $\mathrm{Mgb}^{\prime}$ & $-0.050 \pm 0.019$ & $0.091 \pm 0.008$ & $3.15 \mathrm{E}-10$ & 36 & 10.96 & 0.009 & 0.004 & 0.008 \\
\hline Fe5270' & $0.027 \pm 0.007$ & $0.024 \pm 0.003$ & 0.001 & 36 & 7.27 & 0.004 & 0.004 & 0.001 \\
\hline Fe5335' & $-0.001 \pm 0.013$ & $0.035 \pm 0.006$ & $1.79 \mathrm{E}-05$ & 36 & 6.02 & 0.004 & 0.004 & 0.000 \\
\hline
\end{tabular}

the velocity dispersion. On the other hand, although all the $\alpha$ elements change along the $\sigma$ sequence more than the Fe-peak elements, the variation of the ratio $[\mathrm{Mg} / \mathrm{Fe}]$ is larger than $[\mathrm{X} / \mathrm{Fe}]$ for the rest of $\alpha$ elements.

Although this experiment has been done using the calculated slopes for the LDEG sample, the conclusions are the same for the HDEGs, as the slopes of the metal-sensitive indices in both subsamples are very similar (see Fig. 8). This is not true, however, for the slopes of the age-sensitive indices. In particular, the relation of $\mathrm{H} \beta$ with $\sigma$ is much steeper for the first subsample of galaxies (see Table 7a, and note the striking difference for $\mathrm{H} \beta$ in Fig. 8). For LDEGs, these indices are strongly correlated with $\sigma$, while for HDEGs the statistical significance of the correlation is lower. There are different points of views about the relation of the $\mathrm{H} \beta$ index with velocity dispersion in the literature. While some authors report a strong correlation between these two parameters (e.g., Jørgensen 1997; T98; Caldwell et al. 2003), others find a weak or null correlation (e.g., Mehlert et al. 2003). These discrepancies could be due to differences in the relation as a function of the environment. In fact, the sample of T98 is composed mainly of field and low density environment galaxies, while the sample of Mehlert et al. (2003) consists of galaxies in the Coma cluster. Jørgensen's (1997) sample is more heterogeneous, containing galaxies belonging to 11 different clusters of variable density and field galaxies, while the Caldwell et al. (2003) sample consists of galaxies in the field and in the Virgo cluster, therefore, very similar to our LDEG sample. In general, it seems that all the studies analysing galaxies in low density environments find a correlation between $\mathrm{H} \beta$ and $\sigma$, while those exclusively analysing galaxies in the Coma cluster do not. However, Kuntschner (1998) finds a significant correlation between $\mathrm{H} \beta$ and $\sigma$ in his sample of nearly coeval Fornax galaxies, although he claims that the slope in this relation is driven mainly by variations in metallicity, as the galaxies in the Fornax cluster span a very broad metallicity range. To study the possibility of an age variation along the $\sigma$ sequence, we have derived the change in age that would explain the slope of the $\mathrm{H} \beta-\sigma$ relation, assuming that the age is the only parameter varying with 
Table 7. b) Line-strength indices used in this work as a function of the logarithm of age and metallicity using the models by V06. The last two columns express the required variation in metallicity with velocity dispersion to explain the slopes of the index $-\sigma$ relations, assuming that the slope is due to an exclusive variation of this parameter with the velocity dispersion.

\begin{tabular}{|c|c|c|c|}
\hline \multirow{2}{*}{ Index } & & $\begin{array}{l}\text { (LDEG) } \\
\partial[\mathrm{M} / \mathrm{H}]\end{array}$ & $\begin{array}{l}\text { (HDEG) } \\
\partial[\mathrm{M} / \mathrm{H}]\end{array}$ \\
\hline & & $\overline{\partial \log \sigma}$ & $\overline{\partial \log \sigma}$ \\
\hline$\overline{\mathrm{D} 4000^{\prime}}$ & $-2.7672+0.3817[\mathrm{M} / \mathrm{H}]+0.3724 \log (\mathrm{age})$ & 0.440 & 0.532 \\
\hline $\mathrm{H} \delta_{\Delta}^{\prime}$ & $+1.0737-0.1206[\mathrm{M} / \mathrm{H}]-0.1155 \log (\mathrm{age})$ & 0.403 & 0.507 \\
\hline $\mathrm{H} \delta_{F}^{\prime}$ & $+1.0202-0.0878[\mathrm{M} / \mathrm{H}]-0.1029 \log ($ age $)$ & 0.593 & 0.380 \\
\hline $\mathrm{CN}_{2}$ & $-0.5655+0.1210[\mathrm{M} / \mathrm{H}]+0.0640 \log ($ age $)$ & 1.470 & 1.464 \\
\hline $\mathrm{Ca} 4227^{\prime}$ & $-0.6957+0.0857[\mathrm{M} / \mathrm{H}]+0.0845 \log ($ age $)$ & 0.313 & 0.304 \\
\hline G4300' & $-0.4797+0.0651[\mathrm{M} / \mathrm{H}]+0.0669 \log ($ age $)$ & 0.267 & 0.504 \\
\hline $\mathrm{H} \gamma_{A}^{\prime}$ & $+0.9424-0.0993[\mathrm{M} / \mathrm{H}]-0.1092 \log ($ age $)$ & 0.565 & 0.554 \\
\hline $\mathrm{H} \gamma_{F}^{\prime}$ & $+1.3029-0.1080[\mathrm{M} / \mathrm{H}]-0.1388 \log ($ age $)$ & 0.900 & 0.738 \\
\hline Fe4383' & $-0.4544+0.0907[\mathrm{M} / \mathrm{H}]+0.0583 \log ($ age $)$ & 0.290 & 0.363 \\
\hline $\mathrm{Ca} 4455^{\prime}$ & $-0.1952+0.0509[\mathrm{M} / \mathrm{H}]+0.0282 \log ($ age $)$ & 0.479 & 0.350 \\
\hline $\mathrm{Fe} 4531^{\prime}$ & $-0.1611+0.0374[\mathrm{M} / \mathrm{H}]+0.0252 \log ($ age $)$ & 0.406 & 0.524 \\
\hline C4668' & $-0.1022+0.0734[\mathrm{M} / \mathrm{H}]+0.0171 \log ($ age $)$ & 0.742 & 0.911 \\
\hline $\mathrm{H} \beta^{\prime}$ & $+0.5974-0.0240[\mathrm{M} / \mathrm{H}]-0.0538 \log ($ age $)$ & 1.271 & 0.487 \\
\hline Fe5015' & $-0.0133+0.0374[\mathrm{M} / \mathrm{H}]+0.0097 \log ($ age $)$ & 0.342 & 0.551 \\
\hline $\mathrm{Mgb}^{\prime}$ & $-0.4036+0.0775[\mathrm{M} / \mathrm{H}]+0.0545 \log ($ age $)$ & 1.289 & 1.174 \\
\hline $\mathrm{Fe} 5270^{\prime}$ & $-0.1415+0.0414[\mathrm{M} / \mathrm{H}]+0.0229 \log ($ age $)$ & 0.391 & 0.579 \\
\hline Fe 5335' & $-0.1561+0.0472[\mathrm{M} / \mathrm{H}]+0.0236 \log ($ age $)$ & 0.432 & 0.739 \\
\hline
\end{tabular}

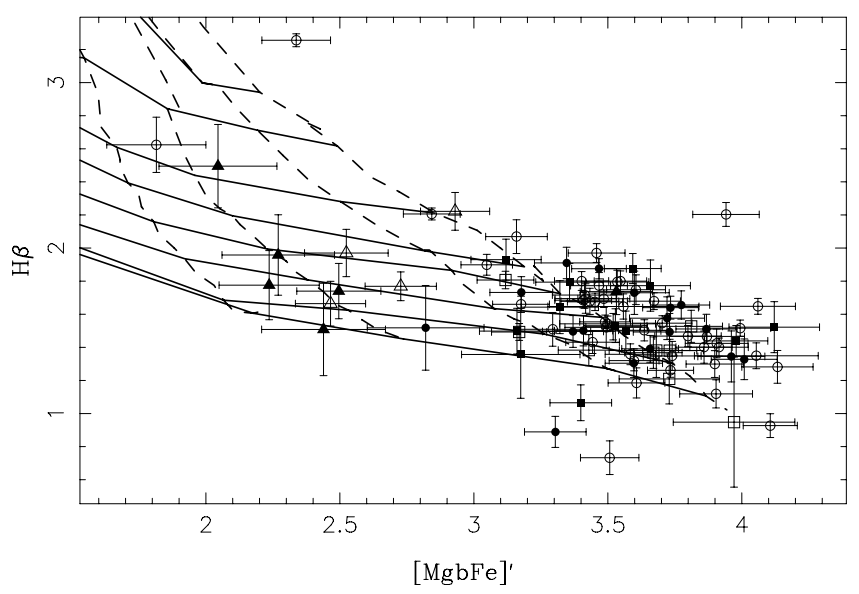

Fig. 7. $[\mathrm{MgFe}]^{\prime}$ versus $\mathrm{H} \beta$ for the LDEG (open symbols) and HDEG (filled symbols). Model grids from V06 are superposed: solid lines are contours of constant age (top to bottom, 1.41, 2.00, 2.82, 3.98, 5.62, $7.98,11.22,15.85 \mathrm{Gyr})$, and dotted lines are contours of constant $[\mathrm{M} / \mathrm{H}]$ (left to right, $[\mathrm{M} / \mathrm{H}]=-0.68,-0.38,+0.00,+0.2)$.

$\sigma$. For the LDEGs, this value is $\partial \log ($ age $) / \partial \log \sigma=0.8$, while for HDEGs is only $\partial \log ($ age $) / \partial \log \sigma=0.2$. In fact, the metallicity variation that explains the slope in the Fe4383- $\sigma$ relation $(\partial[\mathrm{M} / \mathrm{H}] / \partial \log \sigma \sim 0.36)$ can account for the slope of the $\mathrm{H} \beta-\sigma$ relation obtained for HDEGs, without any further variation in age. For the LDEGs an additional age variation of $\partial \log ($ age $) / \partial \log \sigma=0.4$ is, however, required.

Summarising, we conclude that an increase of the overall metallicity with the velocity dispersion for both HDEGs and LDEGs exists. However, not all the elements change in lockstep along the $\sigma$ sequence. The models that best reproduce the slopes in all the indices are those in which the $\alpha$ elements change more than the Fe-peak elements and, furthermore, the $[\mathrm{Mg} / \mathrm{Fe}]$ and $[\mathrm{N} / \mathrm{Fe}]$ ratios change more than the rest of the $\alpha$ elements with the velocity dispersion of the galaxies. In the case of LDEGs, the slopes of the index $-\sigma$ relations also reflect a trend in the mean age of the galaxies in the sense that less massive galaxies are also younger. We will analyse this relation in more detail in Paper II,

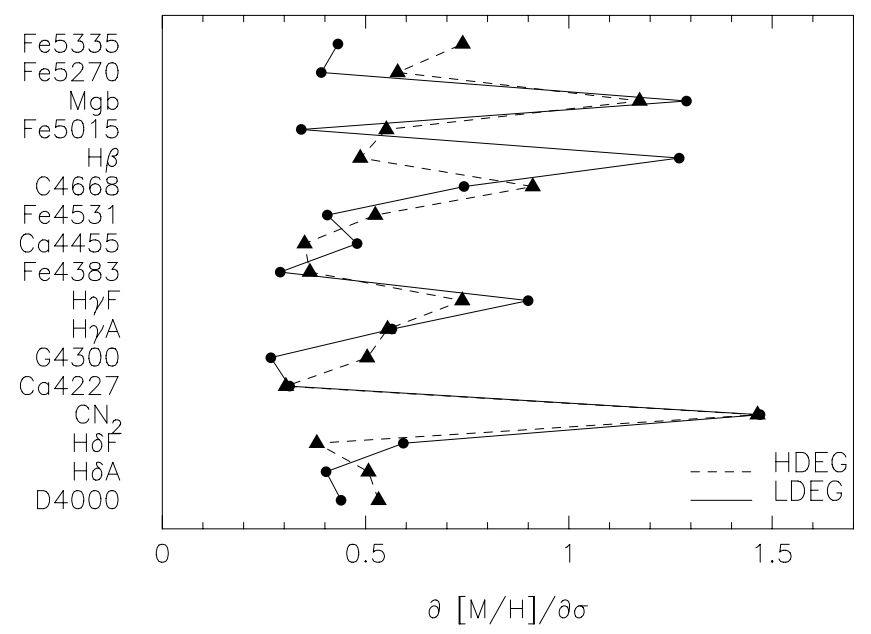

Fig. 8. Variation in metallicity required to explain the slope in the index $-\sigma$ relations for 17 indices, assuming that the metallicity is the only parameter changing with $\sigma$. The solid line represents the change in metallicity for the LDEGs, while the dashed line refers to the galaxies in the Coma cluster.

where we derive ages and metallicities for our sample of galaxies. None of these conclusions would change if we increased the $\mathrm{Mgb}$ and $\mathrm{C} 4668$ indices by a constant value, as all the results are based in the slope of the index- $\sigma$ relations.

\subsection{The dispersion of the relations}

The low dispersion in the $\mathrm{Mg}_{2}-\sigma$ relation and the fact that it is distance independent make it a powerful tool to constrain the models of formation and evolution of galaxies. In fact, the low dispersion has been used as an argument to give evidence that all ellipticals have nearly coeval stellar populations (Bender et al. 1993; Bernardi et al. 1998) in clear contradiction with other studies (G93; T98; Trager et al. 2001; Caldwell et al. 2003). Some authors have studied this problem, concluding that the low scatter is due to a conspiracy between the age and the metallicity, in the sense that younger galaxies are also more metal rich, 


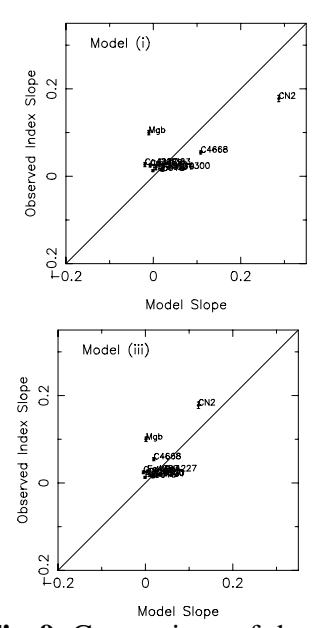

Fig. 9. Comparison of the observed slopes in the index- $\sigma$ relations for the LDEGs ( $y$-axis) with the expected slopes caused by changes in abundance ratio patterns given in the four models discussed in the text ( $x$-axis). The errors bars show the error in the slope of the relations (see text for details).

canceling the deviations with respect to the mean relation (e.g., Worthey et al. 1995; Trager 1997; Pedraz et al. 1998; Jørgensen 1999; K01).

However, though small, an intrinsic dispersion does exists in the $\mathrm{Mg}_{2}-\sigma$ relation. To understand the cause of this scatter, many authors have tried to find correlations between the deviation from the relation and other parameters. Bender et al. (1993) did not find any correlation between the residuals of this relation and other structural parameters of the galaxies such as effective radius, surface brightness, or mass. Neither did they find any correlation between the residuals and the position of the objects in the Fundamental Plane. On the other hand, Schweizer et al. (1990) found a correlation between the deviation of the $\mathrm{Mg}_{2}-\sigma$ relation and the fine structure parameter $\sum$, which is an indicator of recent interactions. González \& Gorgas (1996, hereafter GG96) found an anti-correlation between the residuals and the $\mathrm{H} \beta$ index, and concluded that recent episodes of star formation could explain, at least partially, the present scatter in the relation (see also Worthey \& Collobert 2003).

In this section we investigate the scatter in the relations of the Lick indices with the central velocity dispersion, trying to find which parameters are varying between galaxies with the same velocity dispersion. As we said, several studies suggest that the scatter in the $\mathrm{Mg}-\sigma$ relations is due to variations in the age of the galaxies at a given $\sigma$ through the dilution of the metallic features in a young stellar population. To explore this possibility, we have investigated, following the GG96 analysis, the dependence of the residuals in the index $-\sigma$ relations on the $\mathrm{H} \beta$ index. We performed a $t$-test to study the degree of correlation between them. Table 9 summarises the results. A correlation is considered significant if the $t$ value is higher than 1.96. As can be seen in the table, we confirm the result obtained by GG96 concerning the existence of an strong anti-correlation between the residuals of the $\mathrm{Mgb}-\sigma$ relation and the $\mathrm{H} \beta$ index for the LDEGs, but, on the other hand, we find a positive correlation between the residuals of the Fe4383- $\sigma$ relation and this index. This is illustrated in Fig. 10. If the age were the main parameter responsible for the scatter in the index $-\sigma$ relations, an anti-correlation of the residuals in the Fe4383- $\sigma$ with $\mathrm{H} \beta$ should also be observed, contrary to our findings. Therefore, our result excludes age as the only parameter producing the dispersion in the index $-\sigma$ relations. On the other hand, if only variations of overall metallicity at a given $\sigma$ were responsible for the dispersion, we would also expect the same behaviour in the correlation of the residuals with $\mathrm{H} \beta$ in both indices. Neither of these two parameters can be entirely responsible for the residuals from the relations. Interestingly, in the HDEG subsample, we do not find any correlation between the residuals and the $\mathrm{H} \beta$ index (we only found a marginal correlation for Fe5015 and Fe5335).

Discarding both age and metallicity as the only parameters responsible for the departure of the galaxies from the index $-\sigma$ relations, we investigate if a variation in the relative abundances between galaxies can help to explain the observed scatter. Figure 11 shows the quotient between the metallicities measured in the $\mathrm{Mgb}-\mathrm{H} \beta$ and $\mathrm{Fe} 4383-\mathrm{H} \beta$ diagrams (see Paper II for details) as a function of velocity dispersion, splitting the sample into galaxies older and younger than 7.5 Gyr (according to their position in the $\mathrm{H} \beta-[\mathrm{MgFe}]$ plane, using V06 models). It is clear from this figure that the $[\mathrm{Mg} / \mathrm{Fe}]$ relation with $\sigma$ depends on the luminosity-weighted mean age of the galaxies and, therefore, on their particular star formation history. In particular, older galaxies have, on average, higher $[\mathrm{Mg} / \mathrm{Fe}]$ than the younger ones. In the lower panel of Fig. 11, we have added the galaxies from the Coma cluster. It can be seen that they follow the trend of the older LDEGs, exhibiting, on average, larger $[\mathrm{Mg} / \mathrm{Fe}]$ ratios than younger LDEGs.

These trends indicate that the scatter in the index $-\sigma$ relations is not an exclusive effect of a dispersion in the age, but it is a consequence of the variation of the element abundance ratios with this parameter. Furthermore, we find a tendency for which older galaxies have, on average, higher $[\mathrm{Mg} / \mathrm{Fe}]$ ratios.

Fisher et al. (1995) noted, studying a sample of 7 bright early-type galaxies, a correlation between $[\mathrm{Mg} / \mathrm{Fe}]$ and the age of the galaxies, in the same way we did. These authors, however, did not find a correlation between the residuals of the Mgb- $\sigma$ relation and the $\mathrm{H} \beta$ index. This could be due to the small sample analysed. Thomas et al. (2002) also find a similar relation between the $[\alpha / \mathrm{Fe}]^{2}$ ratio and the age, studying several samples from the literature. Contrary to these findings, Trager et al. (2000b) do not report any correlation between $[\alpha / \mathrm{Fe}]^{3}$ and the age of the galaxies. However these authors analysed the relation between age, $[\mathrm{Z} / \mathrm{Fe}],[\mathrm{E} / \mathrm{Fe}]$ and $\sigma$, concluding that age cannot be the only parameter responsible for the scatter in the $\mathrm{Mg}-\sigma$ relation, being necessary variations of $[\mathrm{E} / \mathrm{Fe}]$ between galaxies with the same $\sigma$, in agreement with our results.

Since $\mathrm{Mg}$ is produced mainly in type II supernovae whilst the bulk of $\mathrm{Fe}$ is released by type I supernovae (e.g., Nomoto et al. 1984; Woosley \& Weaver 1995; Thielemann et al. 1996), the existence of a trend between $[\mathrm{Mg} / \mathrm{Fe}]$ and age favours the idea that the different element ratios are a consequence of different star formation histories (see, e.g., Greggio \& Renzini 1983; Matteucci \& Greggio 1986; Gibson 1997; Thomas et al. 1998), and that younger galaxies have suffered a more extended star formation history, incorporating the elements produced by lowmass stars (see, e.g., Worthey 1998). However, there are other possibilities that we explore later in Sect. 7. Again, in this section, none of the conclusions depends on the absolute values of the indices, therefore, they would not change if we add a constant offset to the C4668 and Mgb indices.

\footnotetext{
${ }^{2}[\alpha / \mathrm{Fe}]$ represents the ratio between the $\alpha$-elements abundances and $\mathrm{Fe}$.

3 These authors do not use this term but instead [E/Fe], being E the abundance of the enhanced elements, which does not coincide completely with the $\alpha$ elements.
} 
Table 8. Difference between the slope of the index $-\sigma$ relation and the expected index variation due to changes in the chemical composition.

\begin{tabular}{lrrrrrrrrrr}
\hline \hline Model & \multicolumn{1}{c}{$\Delta \mathrm{CN}_{2}$} & $\Delta \mathrm{Ca} 4227$ & $\Delta \mathrm{G} 4300$ & $\Delta \mathrm{Fe} 4383$ & $\Delta \mathrm{Ca} 4455$ & $\Delta \mathrm{Fe} 4531$ & $\Delta \mathrm{C} 4668$ & $\Delta \mathrm{Fe} 5015$ & $\Delta \mathrm{Mgb}$ & $\mathrm{rms}$ \\
\hline i & -0.109 & 0.046 & -0.050 & 0.013 & 0.032 & -0.003 & -0.054 & 0.014 & 0.110 & 0.040 \\
ii & -0.148 & 0.055 & -0.068 & 0.039 & 0.030 & 0.004 & -0.050 & -0.050 & 0.092 & 0.045 \\
iii & 0.056 & -0.016 & -0.008 & 0.022 & 0.029 & -0.003 & 0.035 & 0.014 & 0.098 & 0.029 \\
iv & -0.002 & 0.029 & -0.019 & 0.031 & 0.033 & -0.002 & 0.006 & 0.026 & 0.006 & 0.023 \\
\hline
\end{tabular}

Table 9. Statistical analysis of the correlations between the residuals of the index $-\sigma$ relation and the $\mathrm{H} \beta$ index. For a significance level lower than $\alpha=0.05$, a value of $t$ higher than 1.96 confirms the existence of correlation.

\begin{tabular}{lrr}
\hline \hline & LDEG & HDEG \\
Index & \multicolumn{1}{c}{$t$} & \multicolumn{1}{c}{$t$} \\
\hline $\mathrm{D} 4000$ & 1.00 & -1.06 \\
$\mathrm{H} \delta_{A}$ & 0.08 & -0.50 \\
$\mathrm{H} \delta_{F}$ & 2.50 & 0.73 \\
$\mathrm{CN}_{2}$ & -0.85 & 0.42 \\
$\mathrm{C} 4227$ & 0.34 & 0.36 \\
$\mathrm{G} 4300$ & 2.21 & 1.04 \\
$\mathrm{H} \gamma_{A}$ & -0.29 & -0.34 \\
$\mathrm{H} \gamma_{F}$ & 2.07 & -0.04 \\
$\mathrm{Fe} 4383$ & 3.00 & 0.82 \\
$\mathrm{Fe} 4531$ & 2.61 & 0.90 \\
$\mathrm{Ca} 4455$ & 2.93 & 0.56 \\
$\mathrm{C} 4668$ & 1.47 & 0.16 \\
$\mathrm{Fe} 5015$ & 1.32 & 2.23 \\
$\mathrm{Mgb}$ & -4.24 & 0.36 \\
$\mathrm{Fe} 5270$ & 1.97 & 1.50 \\
$\mathrm{Fe} 5335$ & 2.20 & 1.85 \\
\hline
\end{tabular}

We want to finish this section justifying the use of the term $[\mathrm{Mg} / \mathrm{Fe}]$ in our discussion when, in reality, we are just comparing the metallicity measured in a $\mathrm{Fe} 4383-\mathrm{H} \beta$ diagram with the one obtained using the $\mathrm{Mgb}-\mathrm{H} \beta$ combination. Figure 12 shows the values of $Z(\mathrm{Fe} 4383) / Z(\mathrm{Mgb})$ compared with the parameter $[\alpha / \mathrm{Fe}]$ derived from TMB03 models. To obtain these values, we used the indices $\mathrm{Fe} 4383, \mathrm{H} \beta$, and $\mathrm{Mgb}$, and followed an iterative process. We first measured age and metallicities assuming $[\alpha / \mathrm{Fe}]=0$ and obtained a first guess of the age that we used to determine $[\alpha / \mathrm{Fe}]$. With this new value, we calculated, again, age and metallicity and so on, until the derived parameters in two consecutive iterations were consistent within 5\% accuracy. As can be seen in Fig. 12, there is an excellent correspondence between the values of $[\alpha / \mathrm{Fe}]$ derived with TMB03 models and the ratio of metallicities measured using Fe4383 and Mgb with the V06 models. We could have used a transformation to derive $[\alpha / \mathrm{Fe}]$ values from $Z(\mathrm{Fe} 4383) / Z(\mathrm{Mgb})$ values, but several aspects of the models need to be understood before deriving any meaningful number. In any case, we are not making any conclusion based on the absolute value of $[\mathrm{Mg} / \mathrm{Fe}]$. We prefer to use the term $[\mathrm{Mg} / \mathrm{Fe}]$ instead of the most general $[\alpha / \mathrm{Fe}]$ since different $\alpha$-elements may have different behaviours in spheroidal systems, as seems to be the case in the bulge stars of our Galaxy (see, e.g., Fulbright et al. 2005).

\section{Differences in the line-strength indices between LDEGs and HDEGs}

Some studies have analysed the possible differences between the line-strength indices of galaxies in different environments. Guzmán et al. (1992) found systematic variations in the zeropoint of the $\mathrm{Mg}_{2}-\sigma$ relation for a sample of Coma galaxies as a

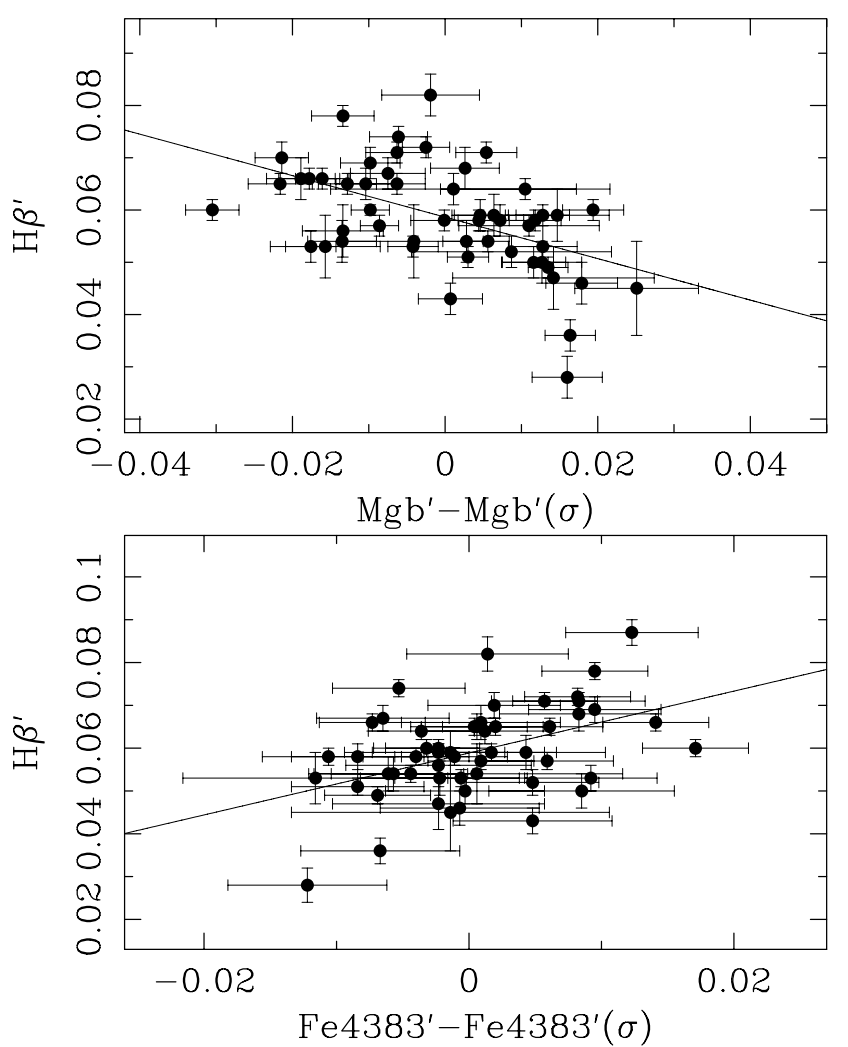

Fig. 10. $\mathrm{H} \beta^{\prime}$ index as a function of the residuals of the $\mathrm{Mgb}^{\prime}-\log \sigma$ $\left(\mathrm{Mgb}^{\prime}-\mathrm{Mgb}^{\prime}(\sigma)\right)$ and $\mathrm{Fe} 4383^{\prime}-\log \sigma\left(\mathrm{Fe} 4383^{\prime}-\mathrm{Fe} 4383^{\prime}(\sigma)\right)$ relations for LDEGs. The linear fits are shown.

function of their distance to the cluster centre. Jørgensen et al. (1996, 1997), examining a sample of eleven clusters, detected a weak correlation between the intensity of the $\mathrm{Mg}$ line and the local density within the clusters, in agreement with the Guzmán et al. result. Similar differences have been found by several other authors (e.g., de Carvalho \& Djorgovski 1992), while others (e.g., Bernardi et al. 1998) do not find evidence of an environmental influence on the zero point of the relation of the indices with the velocity dispersion of the galaxies. The dependence of the absorption features on the environment has been studied, almost exclusively, with just two indices, $\mathrm{Mg}_{2}$ and $\mathrm{Mgb}$.

In this section, we study the differences on 17 Lick indices, at a given $\sigma$, between LDEGs and HDEGs. To quantify possible systematic differences, we have performed a linear leastsquares fit to the index $-\sigma$ relations of the LDEGs subsample (see Sect. 5) and have measured the mean offsets (weighting with errors) of the HDEGs from the fits. Table 10 shows these mean offsets $(d)$ with their errors $\left(s / \sqrt{n_{\mathrm{eff}}}\right.$, where $s$ is the standard deviation and $n_{\mathrm{eff}}$ the effective number of points). We find significant differences in the following indices: $\mathrm{H} \delta_{A}, \mathrm{H} \delta_{F}, \mathrm{Ca} 4455$, $\mathrm{Fe} 4531, \mathrm{Fe} 5015$, and also in the indices $\mathrm{CN}_{2}$ and $\mathrm{C} 4668$, whose differences have been previously reported by Sánchez-Blázquez et al. (2003). On the other hand, in agreement with other studies 


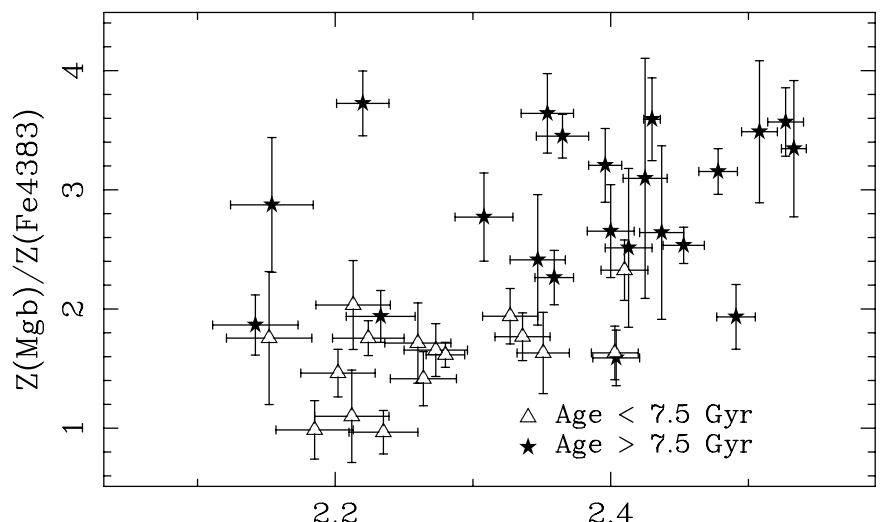

$\log \sigma$

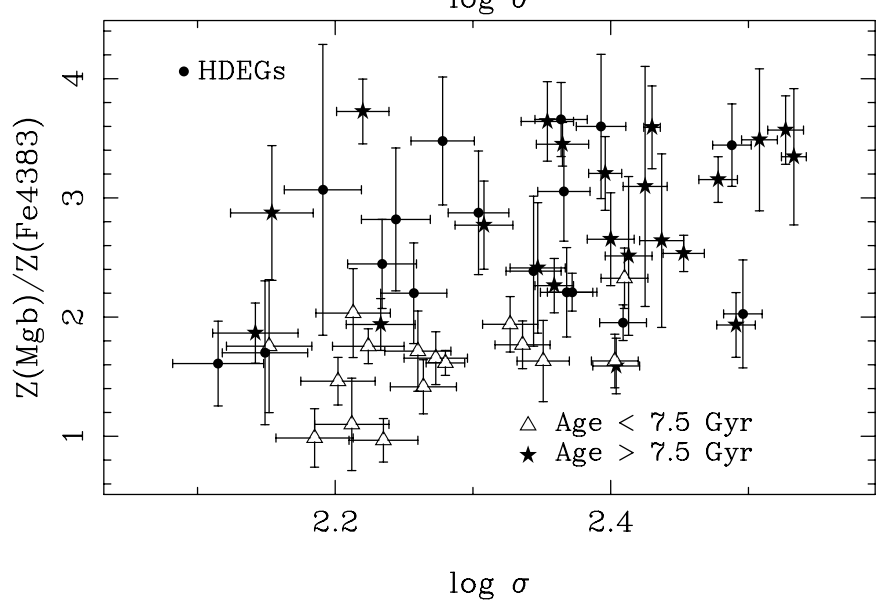

Fig. 11. Ratio of the metallicities calculated in the $\mathrm{Mgb}-\mathrm{H} \beta$ and $\mathrm{Fe} 4383-\mathrm{H} \beta$ diagrams as a function of the velocity dispersion for LDEGs. Different symbols represent galaxies younger (open triangles) and older (filled stars) than $7.5 \mathrm{Gyr}$, as derived from a $[\mathrm{MgFe}]-\mathrm{H} \beta$ diagram. In the lower panel we have also included the sample of HDEGs (solid circles).

(e.g., Bernardi et al. 1998), we do not find any significant difference in the Mgb index of both subsamples at a given velocity dispersion. In the next section, we discuss the possible causes of these differences.

\subsection{Possible interpretations of the differences}

In Sánchez-Blázquez et al. (2003), we briefly discussed possible mechanisms that would produce differences in the $\mathrm{CN}$ and C4668 between galaxies in different environments. In this section, we extend this discussion to include the rest of the indices analysed.

- IMF variations: given the high sensitivity of the $\mathrm{CN}_{2}$ index to the stellar surface gravity (giant stars have higher $\mathrm{CN}_{2}$ index than dwarfs; Gorgas et al. 1993; Worthey et al. 1994), a lower proportion of giant stars in HDEGs with respect to LDEGs would produce systematic differences in that and in some other spectral characteristics between both subsamples. However, $\mathrm{H} \delta_{A}, \mathrm{H} \delta_{F}, \mathrm{C} 4668, \mathrm{Ca} 4455, \mathrm{Fe} 4531$, and $\mathrm{Fe} 5015$ are not especially sensitive to gravity variations. Therefore, it does not seem probable that this is the reason behind the reported differences. In any case, and to quantify this, we calculated the variation of the IMF slope necessary to produce the observed difference in the $\mathrm{CN}_{2}$ index using the predictions of V06 corresponding to a single stellar

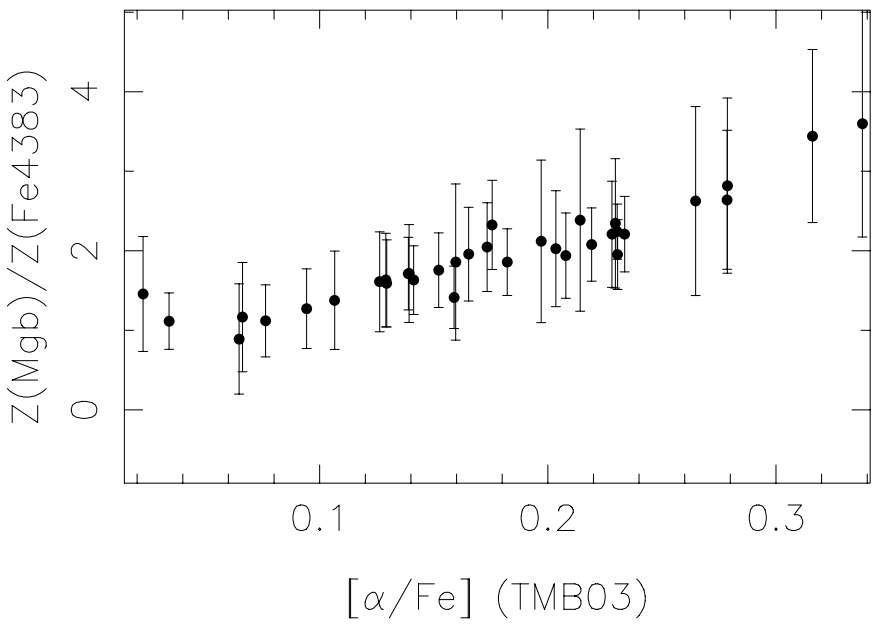

Fig. 12. Comparison of $Z(\mathrm{Fe} 4383) / Z(\mathrm{Mgb})$ with the values of $[\alpha / \mathrm{Fe}]$ derived using the models by TMB03. $Z(\mathrm{Fe} 4383)$ represents the metallicity measured in a $\mathrm{Fe} 4383-\mathrm{H} \beta$ diagram, while $Z(\mathrm{Mgb})$ the metallicity measured in a Mgb-H $\beta$.

Table 10. Mean differences in the Lick indices at a given $\sigma$ between galaxies in different environments. For each index, we list the mean offset $d$ and its error $\Delta d$ and the $t$-parameter (of the statistical test $d \neq 0$ ).

\begin{tabular}{lrcc}
\hline \hline Index & \multicolumn{1}{c}{$d$} & $\Delta d$ & $t$ \\
\hline $\mathrm{D} 4000$ & 0.0067 & 0.0208 & 0.321 \\
$\mathrm{H} \delta_{A}$ & 0.0202 & 0.0041 & 4.904 \\
$\mathrm{H} \delta_{F}$ & 0.0158 & 0.0032 & 4.886 \\
$\mathrm{CN}_{2}$ & -0.0287 & 0.0062 & 4.600 \\
$\mathrm{Ca} 4227$ & -0.0057 & 0.0034 & 1.689 \\
$\mathrm{G} 4300$ & -0.0035 & 0.0034 & 1.121 \\
$\mathrm{H} \gamma_{A}$ & 0.0061 & 0.0033 & 1.843 \\
$\mathrm{H} \gamma_{F}$ & 0.0060 & 0.0042 & 1.442 \\
$\mathrm{Fe} 4383$ & -0.0029 & 0.0022 & 1.332 \\
$\mathrm{Ca} 4455$ & -0.0063 & 0.0020 & 3.166 \\
$\mathrm{Fe} 4531$ & -0.0058 & 0.0015 & 3.827 \\
$\mathrm{C} 4668$ & -0.0103 & 0.0029 & 3.535 \\
$\mathrm{H} \beta$ & 0.0006 & 0.0024 & 0.259 \\
$\mathrm{Fe} 5015$ & -0.0053 & 0.0014 & 3.776 \\
$\mathrm{Mgb}$ & 0.0015 & 0.0029 & 0.500 \\
$\mathrm{Fe} 5270$ & -0.0022 & 0.0010 & 2.146 \\
$\mathrm{Fe} 5335$ & 0.0014 & 0.0014 & 0.993 \\
\hline & & &
\end{tabular}

population of $10 \mathrm{Gyr}$ and solar metallicity. This variation in the slope of the IMF would also reproduce the observed differences in C4668 and Fe5015, but, at the same time, would lead to variations in the opposite sense in the Ca4455 and Fe4531 indices. Therefore, although we do not discard the possibility of differences in the IMF between both subsamples, it cannot be the only factor responsible for the offsets of the relations in the indices.

- Differences in the mean age of galaxies in distinct environments: this would also introduce systematic variations in the line-strength indices. Using V06 models, the observed differences in $\mathrm{CN}_{2}, \mathrm{C} 4668, \mathrm{Ca} 4455, \mathrm{Fe} 4531$, and Fe5015 could be explained by assuming an age discrepancy between both subsamples of $9.8 \mathrm{Gyr}$, with the Coma galaxies being younger than the galaxies in low density environments (considering a single burst with solar metallicity, a Salpeter IMF, and a variation in age from 7.94 to $17.78 \mathrm{Gyr}$ ). This age difference would, however, produce changes in the $\mathrm{H} \delta$ indices 
almost twice as large as observed, and would also produce differences in other indices, like $\mathrm{H} \beta$, not only higher than observed, but in the opposite sense to the measured ones. Furthermore, the results reported by other authors suggest that galaxies in denser environments are older than galaxies in the field and low density groups (e.g., Kuntschner et al. 2002; Sánchez-Blázquez 2004; Thomas et al. 2005; see also Paper II).

- Variations in the relative abundances between LDEG and $H D E G$ : another explanation for the observed offsets could be the existence of differences in the element abundance ratios of different chemical species. According to TB95, C4668 is extremely sensitive to variations in the carbon abundance. $\mathrm{CN}_{2}$, on the other hand, is sensitive to variations of both $\mathrm{C}$ and $\mathrm{N}$ abundances. Furthermore, the blue band of the $\mathrm{H} \delta$ index overlaps with the $\mathrm{CN}$ bands, causing a decrease in these values as the $\mathrm{N}$ abundance increases (Worthey \& Ottaviani 1997; Schiavon et al. 2004). Fe4531 and Fe5015 are both very sensitive to variations of $\mathrm{Ti}$.

If we assume than the differences in $\mathrm{CN}_{2}, \mathrm{C} 4668, \mathrm{Fe} 5015$, and Fe4531 between HDEGs and LDEGs are due to variations in some chemical species with respect to $\mathrm{Fe}$, the existence of differences in $\mathrm{C}, \mathrm{N}$ and $\mathrm{Ti}$ abundances between both subsamples would be required. This is a very qualitative statement. To check if a variation of these chemical species is compatible with the calculated offsets for all the indices, we follow a similar approach to that in Sect. 5.1. In this case, instead of comparing the variation of the indices with the velocity dispersion, we compare the offsets between galaxies in different environments and try to reproduce these differences by varying the abundances of different chemical species. We separated the elements into 6 different groups: (i) $\alpha$ elements: $\mathrm{Ne}, \mathrm{Na}, \mathrm{Mg}, \mathrm{Si}$, $\mathrm{S}$, and $\mathrm{O}$; (ii) $\mathrm{C}$; (iii) $\mathrm{N}$; (iv) $\mathrm{Ti}$; (v) Ca; and (vi) Fe-peak elements ( $\mathrm{Fe}$ and $\mathrm{Cr}$ ). We have built 4 different models. In building the models, we have not tried to fit all the indices by varying the ratios of different elements arbitrarily, but we have proposed 4 simple permutations that can give a rough idea of the differences in the chemical composition between galaxies in different environments. This rough idea, though, can give us important clues to understand the differences in the star formation history of galaxies between LDEG and HDEG. The models can be described as follows:

Model 1: the abundances of $\mathrm{C}$ and $\mathrm{N}$ are increased by 0.05 dex, leaving the other element abundances unchanged.

Model 2: the abundances of $\mathrm{C}$ and $\mathrm{N}$ are increased by $0.05 \mathrm{dex}$, the abundance of Ti by $0.15 \mathrm{dex}$, and the other elements are kept fixed.

Model 3: the abundances of $\mathrm{C}$ is increased by $0.04 \mathrm{dex}, \mathrm{N}$ by 0.05 dex, Ti by 0.15 dex, and Ca by 0.10 dex; the remaining abundances are unchanged.

Model 4: similar to Model 3, increasing the abundances of all the $\alpha$ and the Fe-peak elements by 0.05 dex and the $\mathrm{N}$ abundance by 0.15 dex.

A summary of the 4 models is shown in Table 11.

Figure 13 shows the comparison of the observed offsets between galaxies in different environments and the ones obtained from the different models. The lines in the figures indicate the 1:1 relation (the last column of Table 11 indicates the rms of the dispersion around this relation). From
Table 11. Differences in the abundances of the distinct chemical species used to build the 4 models described in the text. The last column shows the dispersion around the 1:1 relation when comparing the offsets in the indices between galaxies in different environments and the offsets in the indices due to the changes in the chemical species of each model.

\begin{tabular}{lrrrrrrr}
\hline \hline Model & {$[\mathrm{C} / \mathrm{H}]$} & {$[\mathrm{N} / \mathrm{H}]$} & {$[\alpha / \mathrm{H}]$} & {$[\mathrm{G} 2 / \mathrm{H}]$} & {$[\mathrm{Ca} / \mathrm{H}]$} & {$[\mathrm{Ti} / \mathrm{H}]$} & $\mathrm{rms}$ \\
\hline 1 & +0.05 & +0.05 & +0.00 & +0.00 & +0.00 & +0.00 & 0.0066 \\
2 & +0.05 & +0.05 & +0.00 & +0.00 & +0.00 & +0.15 & 0.0060 \\
3 & +0.04 & +0.05 & +0.00 & +0.00 & +0.10 & +0.15 & 0.0035 \\
4 & +0.04 & +0.15 & +0.05 & +0.05 & +0.10 & +0.15 & 0.0034 \\
\hline
\end{tabular}

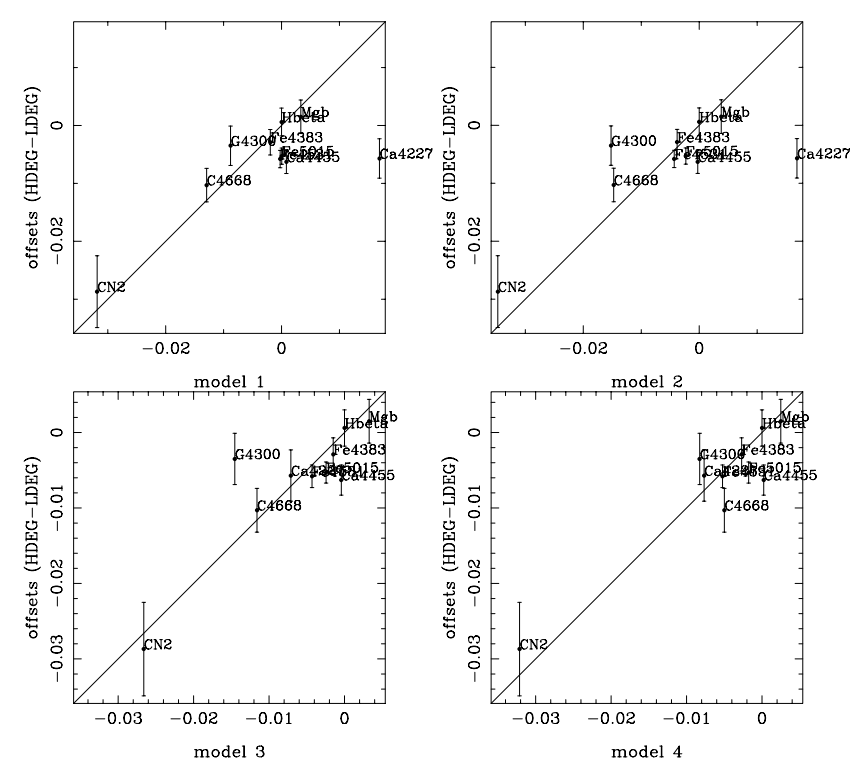

Fig. 13. Comparison of the offsets between HDEGs and LDEGs with the differences in the indices obtained in different models in which we have calculated the variation in the Lick indices due to variations on the ratio of different chemical elements (see text for a more detailed description). The error bars indicate the errors in the offsets.

the analysis of the figure and the rms of the residuals, we can conclude the following:

- The models in which the only elements varying are $\mathrm{C}, \mathrm{N}$, and $\mathrm{Ti}$ are not the best to reproduce the observed offsets in all the indices.

- Although we do not find a significant difference in the Ca4227 index between galaxies in different environments, due to the dependence of this index on the $\mathrm{C}$ abundance (its value drops when the $\mathrm{C}$ abundance increases; see TB95), a difference in the Ca abundance is necessary to reproduce the observed differences in this index.

- The models that include a variation in all the chemical species best reproduce the differences in all the observed indices.

- The observed differences between LDEGs and HDEGs are reproduced with a very small variation in the $\mathrm{C}$ abundance. However, it is difficult to simultaneously explain both the C4668 and G4300 indices. In fact, to reproduce the differences in G4300 a larger difference in the C abundance between low- and high-density environment galaxies would be necessary. Two possibilities, as we argued in Sect. 5.1, are that the index C4668 is not as sensitive to the $\mathrm{C}$ abundance as reported in TB95 or that G4300 is not very well calibrated in the model atmospheres of TB95. 
One study that may provide evidence for the existence of $\mathrm{C}$ differences between galaxies in different subsamples is that of Mobasher \& James (2000). These authors found significant differences in the $\mathrm{CO}$ band at $2.3 \mu \mathrm{m}$ between galaxies in the centre and in the outskirts of the Coma cluster. These authors interpreted the differences as evidence of the presence of a younger population in galaxies situated in the field, due to a larger contribution of AGB stars. Although in the present work we do not discard this possibility, the data presented in this paper allow an interpretation in which the differences are mainly due to changes in the $\mathrm{C}$ abundance. In any case, as we have shown, the reported differences between indices cannot be explained exclusively with an age variation between samples.

In summary, to reproduce the observed differences between the indices of LDEGs and HDEGs through variations in the abundances of different elements, we have to assume a variation in all the chemical species, in the sense that LDEGs are, on average, more metal rich than HDEGs. The relative changes in all the chemical species, however, are not the same. In particular, the models that best explain the differences are those that assume a higher variation in $\mathrm{N}, \mathrm{Ti}, \mathrm{Ca}$, and probably $\mathrm{C}$, between galaxies in different environments. $\mathrm{Ti}$ and $\mathrm{Ca}$ are elements that are very difficult to interpret. $\mathrm{Ca}$ is an $\alpha$-element, but in early-type galaxies seems to track Fepeak elements (Worthey 1998; TMB03; Cenarro et al. 2004). Ti is a poorly understood element. Nucleosynthesis theories predict that this element is produced in type II supernovae and that its abundance is similar to that of Fe. However, in galactic bulge stars, this element is found in higher proportions than in the solar partition, even for stars of solar metallicity (McWillian \& Rich 1994, 2004), which has not been reproduced by any nucleosynthesis model. This element can also be overabundant with respect to $\mathrm{Fe}$ in massive ellipticals (Worthey 1998). So, we do not try to discuss the origin of the differences in these two elements between galaxies in different environments. The conclusions of this section would not change if we were to add a constant offset to the indices $\mathrm{C} 4668$ and $\mathrm{Mgb}$, as the conclusions are based upon differences between galaxies in different environments, not on the absolute values of these indices.

In the following subsection, we extend the discussion already presented in Sánchez-Blázquez et al. (2003) about the possible mechanisms that can lead to a difference in the $[\mathrm{C} / \mathrm{Fe}]$ and $[\mathrm{N} / \mathrm{Fe}]$ ratios between low- and high-density environment galaxies.

\subsection{Abundances of $C$ and $N$}

$\mathrm{C}$ is produced predominantly by the triple-alpha reaction of $\mathrm{He}$, while $\mathrm{N}$ is produced in the conversion of $\mathrm{C}$ and $\mathrm{O}$ during the CNO cycles. The problem is to determine the evolutionary phases in which these elements are predominantly produced. Several studies seem to favour intermediate-mass stars (between 5 and $8 M_{\odot}$ ) as the main contributors to $\mathrm{N}$ (Renzini \& Voli 1981; Henry et al. 2000; Chiappini et al. 2003), although it is also predicted to be produced in massive stars (Meynet \& Maeder 2002). There is still more controversy over the location of the $\mathrm{C}$ production. Some authors claim that this occurs in low- and intermediate-mass stars (Renzini \& Voli 1981; Chiappini et al. 2003a) based on the variation of $[\mathrm{C} / \mathrm{Fe}]$ with metallicity in the stars of the galactic disk. This ratio remains constant from $[\mathrm{Fe} / \mathrm{H}] \sim-2.2$ to solar metallicities, as would be expected if it were produced in non-massive stars. On the other hand, recent measurements of the $\mathrm{C} / \mathrm{O}$ abundances among halo and disk stars show a discontinuity around $\log (\mathrm{O} / \mathrm{H}) \sim-3.6$. Oxygen is mainly created in massive stars; therefore, if the main contributors to the $\mathrm{C}$ abundance were also these stars, we would not expect to find this discontinuity. However, other authors affirm that, to explain the abundances of the observed $\mathrm{C}$ in stars in the galactic disk, most of the $\mathrm{C}$ has to be produced in massive stars (Carigi 2000; Henry et al. 2000). The conclusions of the different authors are very dependent on the adopted yields. The works that favour massive stars as the main contributors to the $\mathrm{C}$ abundance in the interstellar medium are based in the Maeder (1992) yields, which predict a strong dependence of the $\mathrm{C}$ yields in massive stars on the metallicity. However, this work has been improved in the new models of Meynet \& Maeder (2002), which have taken into account the effects due to the stellar rotation. Using these models, Chiappini et al. (2003) showed that, considering massive stars as the main producers of $\mathrm{C}$, it is not possible to explain the solar $\mathrm{C} / \mathrm{O}$ ratio. In this work we consider that $\mathrm{C}$ is produced mainly in low- and intermediate-mass stars $\left(1 \leq M / M_{\odot} \leq 8\right)$, while $\mathrm{N}$ is mainly produced in intermediate mass stars $\left(5 \leq M / M_{\odot} \leq 8\right)$. During the AGB, these stars eject significant amounts of ${ }^{4} \mathrm{He}$, ${ }^{12} \mathrm{C},{ }^{13} \mathrm{C}$, and ${ }^{14} \mathrm{~N}$ into the interestellar medium. Of course, the interpretation is subject to change if new results in stellar nucleosynthesis report that these stars are not the main contributors to these elements.

A difference in the relative abundance of $\mathrm{C}$ and $\mathrm{N}$ between LDEGs and HDEGs may be due to:

- A difference in the yields of the stars in HDEGs and LDEGs.

- A difference in the star formation history between both subsamples.

We start exploring the first possibility. The yields of low- and intermediate-mass stars change with the time between thermal pulses during the AGB, the number of these pulses and the efficiency of the third dredge-up. These factors depend, fundamentally, on the mass of the stars and their metallicity. The number and the duration of the thermal pulses in AGB stars increase with the mass of the star, as do the yields. However, when the mass of the star is higher than 3-4 $M_{\odot}$, a process known as hot bottom burning occurs. This process converts part of the ${ }^{12} \mathrm{C}$ into ${ }^{14} \mathrm{~N}$. The efficiency of this process increases with mass and decreases with metallicity (Marigo 2001) and has a particularly noticeable effect on the yields of ${ }^{12} \mathrm{C}$ and ${ }^{14} \mathrm{~N}$, leading to an increase in $\mathrm{N}$ and a decrease of $\mathrm{C}$ in the interstellar medium. A higher metallicity in HDEG with respect to LDEG could increase the efficiency of this phenomenon in the former, leading to an increase in the $\mathrm{N}$ abundance and a decrease in the $\mathrm{C}$ abundance. The differences in the indices $\mathrm{C} 4668$ and $\mathrm{CN}_{2}$, however, require that both $\mathrm{C}$ and $\mathrm{N}$ be in higher proportions in LDEGs with respect to the galaxies in high density environments. A higher metallicity would also increase the amount of $\mathrm{C}$ in the interstellar medium, due to more efficient mass loss in massive stars (Prantzos et al. 1994). This is caused by an increase in the opacities of the stellar envelopes. Therefore, the more metal rich massive stars would produce more $\mathrm{C}$ than the less massive ones through this mechanism (these stars would also produce less $\mathrm{O}$ and more $\mathrm{He}$ ), which could compensate for the decrease of $\mathrm{C}$ abundance in the hot bottom burning. However, the metallicity of LDEG is, on average, higher than in HDEGs (see Paper II), which is the opposite of what is needed to explain the observed variations between 
galaxies in distinct environments through differences in the stellar yields. Another effect that can change the atmospheric abundances in the stars is mixing due to the rotation of the star (Norris 1981; Sweigart 1997). While it does not have a big effect in the ${ }^{4} \mathrm{He}$, it may be important for the ${ }^{12} \mathrm{C}$ (especially in low-mass stars) and ${ }^{14} \mathrm{~N}$ yields. Following Meynet $\&$ Maeder (2002), rotation increases the size of the $\mathrm{C}$ and $\mathrm{O}$ core, due to an increase of the mixing efficiency. The effect is particularly important at low metallicities, where the angular velocity gradient is higher. In any case, it is hard to imagine why the rotation of the stars should be different in galaxies inhabitating environments of different galaxy density.

The second mechanism that could produce variations in the chemical abundances of $\mathrm{C}$ and $\mathrm{N}$ between galaxies in different environments is a difference in their star formation history. In particular, if we assumed that the main contributors to the $\mathrm{C}$ and $\mathrm{N}$ are the intermediate- and low-mass stars, the timescale for the release of these elements into the interstellar medium would be $\sim 3 \times 10^{7}$ years (this is, approximately, the lifetime of an $8 M_{\odot}$ star). Therefore, if star formation in the HDEGs was shorter than this, the stars would not incorporate those elements. However, if the timescale of the star formation in LDEGs were long enough for stars between 3 and $8 M_{\odot}$ to complete their evolution until the AGB, and to release the products of their nucleosynthesis into the interstellar medium, the next generation of stars would incorporate those elements, which would produce the observed differences between both subsamples (LDEGs and HDEGs). These differences in the star formation timescales were proposed in Sect. 5.2 to explain the trends between $\mathrm{Z}(\mathrm{Mgb}) / \mathrm{Z}(\mathrm{Fe} 4383)$ and age. Note that the ages and metallicities that we are measuring are mean values weighted with the luminosity of the stars. Therefore, it is not required that all the stars of the LDEGs are formed more recently, since the differences could be produced by a small percentage of stars formed in later bursts (see Trager et al. 2000b).

\section{The relation between $[\alpha / \mathrm{Fe}]$ and age}

In Sect. 5.2 we showed that the $[\mathrm{Mg} / \mathrm{Fe}]$ ratio is related to the age in such a way that older galaxies exhibit, on average, larger $[\mathrm{Mg} / \mathrm{Fe}]$ ratios than the younger ones.

Several different mechanisms have been proposed to explain the existence, in giant elliptical galaxies, of an overabundance of $[\mathrm{Mg} / \mathrm{Fe}]$ (see, for example, Worthey et al. 1992). All are based on the assumption that this parameter quantifies the relative importance of the chemical enrichment from type II versus the delayed type Ia supernovae. We assess whether these proposed mechanisms can explain the relation between the age and the relative abundance of $\mathrm{Mg}$ with respect to $\mathrm{Fe}$.

- Variations of the IMF: a shallower slope of the IMF would lead to a larger fraction of massive stars and, therefore, to a larger fraction of type II supernovae. We could explain the variation of $[\mathrm{Mg} / \mathrm{Fe}]$ with age under this scenario, assuming that the slope of the IMF increases over time. Galaxies that formed their stars earlier, therefore, would contain more massive stars compared with galaxies that formed their stars in a more recent epoch. The evolution of the IMF with time was proposed to explain the bimodal star formation of our Galaxy (Schmidt 1963). Under this scenario, the first generation of stars enriched the interstellar medium very quickly, out of which other stars formed, with a mass distribution compatible with a much steeper IMF. The first author to

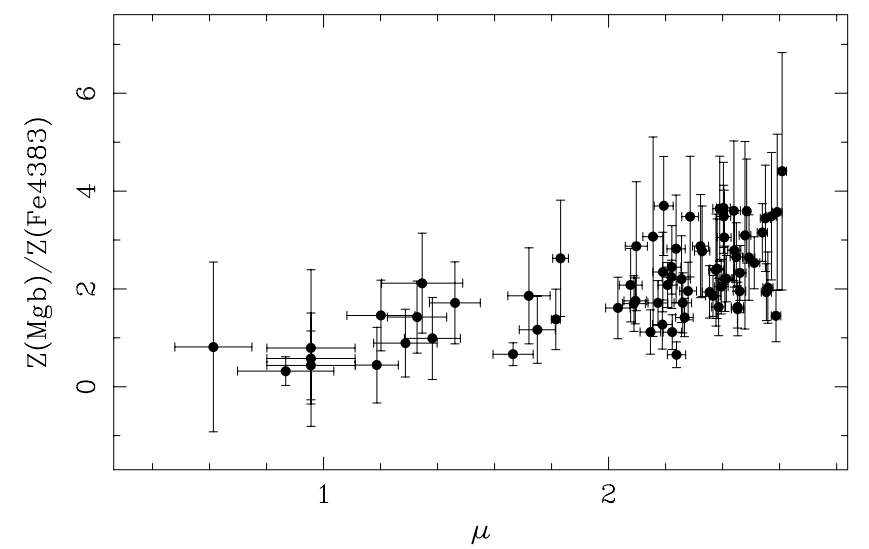

Fig. 14. Slope of the IMF $(\mu)$ derived from the relation between this parameter and $\sigma$ calculated by Cenarro et al. (2003) versus the quotient between the metallicities determined from Mgb and Fe4383.

propose an IMF varying with time was Schmidt (1963), but several authors have analysed this idea (Arnaud et al. 1992; Worthey et al. 1992; Elbaz et al. 1995; Vazdekis 1999, among others).

To explore the possibility of a variation of the IMF slope with $\sigma$, we made use of the Vazdekis et al. (2003) stellar population models. In these models, a new calibration of the $\mathrm{CaT}$ index in the near-infrared derived from a new stellar library (Cenarro et al. 2002) was presented. These authors found that this index has a high sensitivity to the slope of the IMF. They also analysed a sample of galaxies with high quality observed spectra (Cenarro et al. 2003), proposing the existence of a variation of the IMF with metallicity, in the sense that the larger the metallicity, the larger the IMF slope. This dependence of the IMF on metallicity would give rise to the observed tendency of $[\mathrm{Mg} / \mathrm{Fe}]$ with age, if there were a relation between age and metallicity, i.e., younger galaxies were also more metal rich. This relation has been observed in several studies (Trager et al. 2000b; K01 among others), although it is difficult to separate the real trend from the one caused by the correlated errors in both parameters (see, e.g., $\mathrm{K} 01)$. A discussion of this relation is presented in Paper II. Figure 14 shows the relation between the slope of the IMF derived from the relation given in Cenarro et al. (2003) to the $\mathrm{Z}(\mathrm{Mgb}) / \mathrm{Z}(\mathrm{Fe} 4383)$ ratio (the quotient between the metallicity measured separately with both Mgb and Fe4383 combined with $\mathrm{H} \beta$, which can be used as an estimation of behaviour (not the numerical value) of the $[\mathrm{Mg} / \mathrm{Fe}]$ ratio (see Sect. 5.2). As can be seen, the slope of the IMF obtained in this way is higher for galaxies with larger $[\mathrm{Mg} / \mathrm{Fe}]$ ratios, which is the opposite of the expected trend. Therefore, although we do not discard the possibility of differences in the IMF between galaxies, they cannot be the responsible for the relation between $[\mathrm{Mg} / \mathrm{Fe}]$ abundance and age.

- Selective loss mechanisms: the gas losses due to supernova winds in the earlier phases of galaxy formation is another mechanism proposed to explain the overabundances of $[\mathrm{Mg} / \mathrm{Fe}]$ in elliptical galaxies (see, for example, Worthey et al. 1992). To produce an overabundance of $[\mathrm{Mg} / \mathrm{Fe}]$, the mechanism has to be selective, so $\mathrm{Mg}$ has to be retained with a higher efficiency than Fe. Powerful starbursts in giant ellipticals could drive out all previously accumulated Fe-rich gas. The next generations of stars would then be preferentially $\mathrm{Mg}$-rich. In any case, there is no reason to suggest that 
gas loss should be more efficient in older galaxies than in younger ones.

- Differences in the star formation history of the galaxies: the last possibility is that the differences in the chemical abundance ratios are due to variations in the star formation histories. Under this scenario, primeval, genuinely old ellipticals preserve the $\alpha$-element overabundances attained at their formation epoch (which should in turn be mainly controlled by the depth of the potential well). On the other hand, galaxies that have experienced subsequent star formation episodes (that is, those that exhibit younger mean ages) have been able to incorporate the later created $\mathrm{Fe}$ into their stars, lowering their $[\mathrm{Mg} / \mathrm{Fe}]$ ratio. This scenario naturally explains the relation between $[\mathrm{Mg} / \mathrm{Fe}]$ abundances and the age of the galaxies, and therefore is our preferred one. Under this scenario, the differences in the $\mathrm{Mg} / \mathrm{Fe}$ ratios between LDEG and HDEG are due to differences in the star formation history, where galaxies in dense clusters have suffered a truncation of their star formation at early epochs. This was already proposed to explain the differences in other chemical species by Sánchez-Blázquez et al. (2003). Under this scenario we would expect to find an age-metallicity relationship in which the younger galaxies should also be more metal rich. We will analyse this relation in Paper II.

\section{Conclusions}

In this paper we have investigated the relations between linestrength indices and velocity dispersion with the aim of understanding their origin, the causes of the scatter, and the influence of environment. The main results can be summarised as follows:

1. The slopes in the index $-\sigma$ relations are mostly due to an increase in metallicity with the velocity dispersion of the galaxies. However, different chemical elements do not vary in lockstep along the $\sigma$ sequence. In particular, $\alpha$ elements vary more than Fe-peak elements. On the other hand, the necessary variations of $\mathrm{N}$ and $\mathrm{Mg}$ to explain the slope in the relations are even larger than those required by the rest of $\alpha$ elements.

2. To explain the slope of the relation of $\mathrm{H} \beta$ with the velocity dispersion obtained for the LDEGs, a variation of the age with the velocity dispersion is required in the sense that low$\sigma$ galaxies also have to be younger. This age variation is not required to explain the slope of the HDEGs.

3. Studying the residuals of the relations of the indices with the velocity dispersion, we conclude that they are due to a variation of the relative abundance of $[\mathrm{Mg} / \mathrm{Fe}]$ with the age of the galaxies, in the sense that older galaxies show, on average, a higher content of $\mathrm{Mg}$ with respect to Fe. This relation is interpreted in terms of different histories of star formation. If the younger galaxies have had a more extended star formation history, the low mass stars have had time to release the products of their nucleosynthesis into the interstellar medium out of which new stars are formed.

4. We have detected differences in some indices between galaxies belonging to distinct environments. These variations are likely a consequence of changes in the overall metallicity, plus differences in the abundances ratios of $\mathrm{Ti}, \mathrm{Ca}, \mathrm{N}$, and probably $\mathrm{C}$, between galaxies in different environments.

All the differences between galaxies in distinct environments can be explained under a common scenario in which galaxies in dense clusters have suffered a more truncated star formation history than their counterparts in low density environments. We will analyse this in more detail in the second paper of the series, where we will derive ages and metallicities by comparing the line-strength indices with synthesis stellar population models.

Acknowledgements. We would like to thank Brad Gibson, Daisuke Kawata and Christopher Thom for their careful reading of the manuscript and for their useful comments. We are also grateful to Javier Cenarro, Reynier Peletier, and Alexandre Vazdekis for fruitful discussions. We are very grateful to the referee, Jim Rose, for his very constructive report and many useful suggestions. The WHT is operated on the island of La Palma by the Royal Greenwich Observatory at the Observatorio del Roque de los Muchachos of the Instituto de Astrofísica de Canarias. The Calar Alto Observatory is operated jointly by the Max-Planck Institute für Astronomie, Heidelberg, and the Spanish Instituto de Astrofísica de Andalucía (CSIC). This research has made use of the SIMBAD database operated at CDS, Strasbourg, France. This work was supported by the Spanish research project AYA 2003-01840, the European Community through its NOVA fellowship program, and the Australian Research Council. We are grateful to the CATs for generous allocation of telescope time.

\section{References}

Arnaud, M., Rothenflug, R., Boulade, O., Vigroux, L., \& Vangioni-Flam, E. 1992, A\&A, 254, 49

Bender, R. 1990, A\&A, 229, 441

Bender, R., Burstein, D., \& Faber, S. M. 1993, ApJ, 411, 153

Bender, R., Saglia, R. P., Ziegler, B., et al. 1998, ApJ, 493, 529

Bernardi, M., Renzini, A., \& da Costa, G., et al. 1998, ApJ, 508, L143

Bernardi, M., Sheth, R. K., \& Annis, J., et al. 2003, AJ, 125, 1866

Bica, E., \& Alloin, D. 1987, A\&A, 181, 270

Binggeli, B., Sandage, A., \& Tammann, G. A. 1985, AJ, 90, 1681

Binggeli, B., Popescu, C. C., \& Tammann, G. A. 1993, A\&AS, 98, 275

Bower, R. G., Ellis, R. S., Rose, J. A., \& Sharples, R. M. 1990, AJ, 99, 530

Bruzual, G. A. 1983, ApJ, 273, 105

Burstein, D., Faber, S. M., Gaskell, C. M., \& Krumm, N. 1984, ApJ, 287, 586

Caldwell, N. 1984, PASP, 96, 287

Caldwell, N., Rose, J. A., \& Concannon, K. D. 2003, AJ, 125, 2891

Cardiel, N. 1999, Ph.D. Thesis, Universidad Complutense de Madrid

Cardiel, N., Gorgas, J., \& Aragón-Salamanca, A. 1995, MNRAS, 277, 502

Carigi, L. 2000, RMxAA, 36, 171

Cenarro, A. J., Gorgas, J., Cardiel, N., Vazdekis, A., \& Peletier, R. F. 2002, MNRAS, 329, 863

Cenarro, A. J., Gorgas, J., Vazdekis, A., Cardiel, N., \& Peletier, R. F. 2003, MNRAS, 339, L12

Cenarro, A. J., Sánchez-Blázquez, P., Cardiel, N., \& Gorgas, J. 2004, ApJ, 614, L101

Chiappini, C., Romano, D., \& Matteucci, F. 2003a, MNRAS, 339, 63

Chiappini, C., Matteucci, F., \& Meynet, G. 2003b, A\&A, 410, 257

Chiosi, C., \& Carraro, G. 2002, MNRAS, 335, 335

Colless, M., Burstein, D., \& Davies, R. L., et al. 1999, MNRAS, 303, 813

Concannon, K. D., Rose, J. A., \& Caldwell, N. 2000, ApJ, 536, L19

de Carvalho, R. R., \& Djorgovski, S. 1992, ApJ, 389, 49

de Lucia, G., Springel, V., White, S. D. M., Croton, D., \& Kauffmann, G. 2006, MNRAS, 366, 499

Denicoló, G., Terlevich, R., \& Terlevich, E., et al. 2005a, MNRAS, 356, 1440 (D05a)

Denicoló, G., Terlevich, R., Terlevich, E., Forbes, D. A., \& Terlevich, A. 2005b, MNRAS, 358, 813

de Vaucouleurs, G. 1961, ApJS, 6, 213

de Vaucouleurs, G., de Vaucouleurs, A., Corwin, H. G., \& Buta, R. J. 1991, Third Reference Catalogue of Bright Galaxies (New York: Springer-Verlag)

de Zeeuw, P. T., Bureau, M., \& Emsellem, E., et al. 2002, MNRAS, 329, 513

Diaferio, A., Kauffmann, G., \& Balogh, M. L., et al. 2001, MNRAS, 323, 999

Djorgovski, S., \& Davis, M. 1987, ApJ, 313, 59

Elbaz, D., Arnaud, M., \& Vangioni-Flam, E. 1995, A\&A, 303, 345

Ellis, R. 1998, Nature, 395, 3

Faber, S. M., Trager, S. C., González, J. J., \& Worthey, G. 1995, in Stellar population, ed. P. C. van der Kruit, \& G. Gilmore (Dordrecht: Kluwer Publisher), IAU Symp., 164, 249

Ferreras, I., Charlot, S., \& Silk, J. 1999, ApJ, 521, 81

Fisher, D., Franx, M., \& Illingworth, G. 1995, ApJ, 448, 119

Forbes, D. A., Ponman, T. J., \& Brown, R. J. N. 1998, ApJ, 508, L43

Fulbright, J. P., Rich, R. M., \& McWilliam, A. 2005, Nuclear Physics A, 758, 197

García, A. M. 1993, A\&AS, 100, 470 
Gavazzi, G., Boselli, A., Scodeggio, M., Pierini, D., \& Belsole, E. 1999, MNRAS, 304, 595

Gebhardt, K., Faber, S. M., \& Koo, D., et al. 2003, ApJ, 597, 239

Gibson, B. K. 1997, MNRAS, 290, 471

Godwin, J. G., Metcalfe, N., \& Peach, J. V. 1983, MNRAS, 202, 113

González, J. J. 1993, Ph.D. Thesis, University of California, Santa Cruz (G93)

González, J. J., \& Gorgas, J. 1996, in Fresh views of elliptical galaxies, ed. A. Buzzoni, \& A. Renzini, ASP Conf. Ser., 86, 225 (GG96)

Gorgas, J., Efstathiou, G., \& Aragón-Salamanca, A. 1990, MNRAS, 245, 217

Gorgas, J., Faber, S. M., \& Burstein, D., et al. 1993, ApJS, 86, 153

Gorgas, J., Cardiel, N., Pedraz, S., \& González, J. J. 1999, A\&AS, 139, 29

Goudfrooij, P., Hansen, L., Jørgensen, H. E., \& Norgaard-Nielsen, H. U. 1994, A\&AS, 105, 341

Greggio, L. 1997, MNRAS, 285, 151

Greggio, L., \& Renzini, A. 1983, A\&A, 118, 217

Guzmán, R., Lucey, J. R., Carter, D., \& Terlevich, R. J. 1992, MNRAS, 257, 187

Henry, R. B. C., Edmunds, M. G., \& Köppen, J. 2000, ApJ, 541, 660

Jørgensen, I. 1997, MNRAS, 288, 161

Jørgensen, I. 1999, MNRAS, 306, 607

Jørgensen, I., Franx, M., \& Kjærgaard, P. 1996, MNRAS, 280, 167

Kauffmann, G. 1996, MNRAS, 281, 487

Kauffmann, G., \& Charlot, S. 1998, MNRAS, 297, L23

King, D. L. 1985, in RGO-La Palma Technical Notes, No. 31

Kobayashi, C., \& Arimoto, N. 1999, ApJ, 527, 573

Kuntschner, H. 1998, Ph.D. Thesis, University of Durham

Kuntschner, H. 2000, MNRAS, 315, 184

Kuntschner, H., \& Davies, R. L. 1998, MNRAS, 295, L29

Kuntschner, H., Lucey, J. R., Smith, R. J., Hudson, M. J., \& Davies, R. L. 2001, MNRAS, 323, 615 (K01)

Kuntschner, H., Smith, R. J., \& Colless, M., et al. 2002, MNRAS, 337, 172

Laird, J. B., \& Levison, H. F. 1985, AJ, 90, 2652

Larson, R. B., Tinsley, B. M., \& Caldwell, C. N. 1980, ApJ, 237, 692

Maeder, A. 1992, A\&A, 264, 105

Marigo, P. 2001, A\&A, 370, 194

Matteucci, F. 2003, Ap\&SS, 284, 539

Matteucci, F., \& Greggio, L. 1986, A\&A, 154, 279

McWilliam, A., \& Rich, R. M. 1994, 91, 749

McWilliam, A., \& Rich, R. M. 2004, in Origin and Evolution of the Elements, from the Carnegie Observatories Centennial Symposia, ed. A. McWilliam, \& M. Rauch, Pasadena, Carnegie Observatories

Mehlert, D., Saglia, R. P., Bender, R., \& Wegner, G. 2000, A\&AS, 141, 449 (M00)

Mehlert, D., Thomas, D., Saglia, R. P., Bender, R., \& Wegner, G. 2003, A\&A, 407,423

Meynet, G., \& Maeder, A. 2002, A\&A, 390, 561

Mobasher, B., \& James, P. A. 1996, MNRAS, 316, 507

Moore, S. A. W., Lucey, J. R, Kuntschner, H., \& Colless, M. 2002, MNRAS, 336, 382 (M02)

Nomoto, K., Thielemann, F. K., \& Wheeler, J. C. 1984, ApJ, 279, 23

Norris, J. 1981, ApJ, 248, 177

Oke, J. B. 1990, AJ, 99, 1621

Pedraz, S., Gorgas, J., Cardiel, N., \& Guzmán, R. 1998, Ap\&SS, 263, 159

Pedraz, S., Gorgas, J., Cardiel, N., Sánchez-Blázquez, P., \& Guzmán, R. 2002, MNRAS, 332, L59

Phillips, M. M., Jenkins, C. R., Dopita, M. A., Sadler, E. M., \& Binette, L. 1986, AJ, 91, 1062

Pickles, A. J. 1985, ApJ, 296, 340

Poggianti, B. M., Bridges, T. J., et al. 2001a, ApJ, 562, 689

Poggianti, B. M., Bridges, T. J., \& Carter, D., et al. 2001b, ApJ, 563, 118

Prantzos, N., Vangioni-Flam, E., \& Chauveau, S. 1994, A\&A, 285, 132

Proctor, R. N., \& Sansom, A. E. 2002, MNRAS, 333, 517

Proctor, R. N., Forbes, D. A., \& Hau, G. K. T. 2004, MNRAS, 349, 1381

Prugniel, Ph., Golev, V., \& Maubon, G. 1999, A\&A, 346, L25

Renzini, A., \& Voli, M. 1981, A\&A, 94, 175

Rose, J. A. 1985, AJ, 90, 1927
Rose, J. A., Bower, R. G., Caldwell, N., et al. 1994, AJ, 108, 2054

Rusin, D., Kochanek, C. S., \& Falco, E. E. 2003, ApJ, 587, 143

Sánchez-Blázquez, P. 2004, Ph.D. Thesis, Universidad Complutense de Madrid

Sánchez-Blázquez, P., Gorgas, J., Cardiel, N., Cenarro, A. J., \& González, J. J. 2003, ApJ, 590, L91

Sánchez-Blázquez, P., Peletier, R. F., Jimenez-Vicente, J., et al. 2006, MNRAS [arXiv: astro-ph/0607009]

Sargent, W. L. W., Schechter, P. L., Boksenberg, A., \& Shortridge, K. 1977, ApJ, 212,326

Savage, B. D., \& Mathis, J. S. 1979, ARA\&A, 17, 73

Schiavon, R. P., Caldwell, N., \& Rose, J. A. 2004, AJ, 127, 1513

Schmidt, M. 1963, ApJ, 137, 758

Schweizer, F., Seitzer, P., \& Faber, S. M., et al. 1990, ApJ, 364, L33

Somerville, R. S., \& Primack, J. R. 1999, MNRAS, 310, 1087

Smail, I., Kuntschner, H., \& Kodama, T. 2001, MNRAS, 323, 839

Sweigart, A. V. 1997, ApJ, 474, L23

Terlevich, R., Davies, R. L., Faber, S. M., \& Burstein, D. 1981, MNRAS, 196, 381

Terlevich, A. I., Kuntschner, H., Bower, R. G., Caldwell, N., \& Sharples, R. M. 1999, MNRAS, 310, 445

Thielemann, F. K., Nomoto, K., \& Hashimoto, M. A. 1996, ApJ, 460, 408

Thomas, D., Greggio, L., \& Bender, R. 1998 MNRAS, 296, 119

Thomas, D., Maraston, C., \& Bender, R. 2002, Ap\&SS, 281, 371

Thomas, D., Maraston, C., \& Bender, R. 2003, MNRAS, 343, 279 (TMB03)

Thomas, D., Maraston, C., Bender, R., \& de Oliveira, C. M. 2005, ApJ, 621, 673

Trager, S. C. 1997, Ph.D. Thesis, University of California, Santa Cruz

Trager, S. C., Worthey, G., Faber, S. M., Burstein, D., \& González, J. J. 1998, ApJS, 116, 1 (T98)

Trager, S. C., Faber, S. M., Worthey, G., \& González, J. J. 2000a, AJ, 119, 1645 (T00a)

Trager, S. C., Faber, S. M., Worthey, G., \& González, J. J. 2000b, AJ, 120, 165

Trager, S. C., Faber, S. M., \& Dressler, A. 2001, in Astrophysical Ages and Times Scales, ASP Conf. Ser., 245, 429

Treu, T., Stiavalli, M., Casertano, S., Møller, P., \& Bertin, G. 1999, MNRAS, 308,1037

Treu, T., Stiavelli, M., Bertin, G., Casertano, S., \& Møller, P. 2001, MNRAS, 326,237

Treu, T., Stiavelli, M., Casertano, S., Møller, P., \& Bertin, G. 2002, ApJ, 564, L13

Treu, T., Ellis, R. S., Liao, T. X., et al. 2005, ApJ, 633, 174

Tripicco, M. J., \& Bell, R. A. 1995, AJ, 110, 3035 (TB95)

Toomre, A. 1977, in Evolution of galaxies and stellar population, ed. B. M. Tinsley, \& R. Larson, New Haven, Yale University Observatory, 401

van Dokkum, P. G., \& Ellis, R. S. 2003, ApJ, 592, L53

van Dokkum, P. G., Franx, M., Kelson, D., Illingworth, G., \& Fabricant, D. 2001, in Proceedings of the ESO/ECF/STScI Workshop, ed. A. Renzini, \& R. E. Williams, Srpinger, 194

Vazdekis, A. 1999, ApJ, 513, 224

Vazdekis, A., Cenarro, A. J., Gorgas, J., Cardiel, N., \& Peletier, R. F. 2003, MNRAS, 340, 1317

Visvanathan, N., \& Sandage, A. 1977, ApJ, 216, 214

Worthey, G. 1994, ApJ, 95, 107

Worthey, G. 1998, PASP, 110, 888

Worthey, G. 2004, AJ, 128, 2826

Worthey, G., \& Ottaviani, D. L. 1997, ApJS, 111, 377

Worthey, G., \& Collobert, M. 2003, ApJ, 586, 17

Worthey, G., Faber, S. M., \& González, J. J. 1992, ApJ, 398, 69

Worthey, G., Faber, S. M., González, J. J., \& Burstein, D. 1994, ApJS, 94, 687

Worthey, G., Trager, S. C., \& Faber, S. M. 1995, in Fresh views of elliptical galaxies, ASP Conf. Ser., 86, 203

Woosley, S. E., \& Weaver, T. A. 1995, ApJS, 101, 181

Yi, S. K., Yoon, S.-J., \& Kaviraj, S., et al. 2005, ApJ, 619, L111

Zwicky, F., Herzog, E., Wild, P., Karpowicz, M., \& Kowal, C. T. 1961, AJ, 79, 1356 
P. Sánchez-Blázquez et al.: Stellar populations of early-type galaxies in different environments. I., Online Material p 1

\section{Online Material}


P. Sánchez-Blázquez et al.: Stellar populations of early-type galaxies in different environments. I., Online Material p 2

\section{Appendix}

\section{Appendix A: Comparison with the Lick library}

Figure A.1 shows, for the stars observed in the different runs, the comparison between the original Lick/IDS index measurements and the indices determined from our data. Table A.1 shows the offsets obtained in the different observing periods and the final offset adopted in this paper.
Table A.1. Final Lick/IDS offsets (Lick/IDS - This work).

\begin{tabular}{lr}
\hline \hline Index & Offset (Lick-this work) \\
\hline $\mathrm{H}_{\delta A}$ & $0.000 \pm 0.227 \AA$ \\
$\mathrm{H}_{\delta F}$ & $0.000 \pm 0.130 \AA$ \\
$\mathrm{CN}_{2}$ & $-0.014 \pm 0.011 \mathrm{mag}$ \\
$\mathrm{Ca} 4227$ & $-0.193 \pm 0.117 \AA$ \\
$\mathrm{G} 4300$ & $-0.346 \pm 0.160 \AA$ \\
$\mathrm{H}_{\gamma A}$ & $0.568 \pm 0.229 \AA$ \\
$\mathrm{H}_{\gamma F}$ & $0.451 \pm 0.116 \AA$ \\
$\mathrm{Fe} 4383$ & $0.000 \pm 0.303 \AA$ \\
$\mathrm{Ca} 4455$ & $0.201 \pm 0.132 \AA$ \\
$\mathrm{Fe} 4531$ & $0.000 \pm 0.128 \AA$ \\
$\mathrm{C} 4668$ & $-0.682 \pm 0.250 \AA$ \\
$\mathrm{H}_{\beta}$ & $-0.104 \pm 0.097 \AA$ \\
$\mathrm{Fe} 5015$ & $0.000 \pm 0.162 \AA$ \\
$\mathrm{Mgb}$ & $-0.157 \pm 0.081 \AA$ \\
$\mathrm{Fe} 5270$ & $0.000 \pm 0.075 \AA$ \\
$\mathrm{Fe} 5335$ & $0.000 \pm 0.150 \AA$
\end{tabular}


P. Sánchez-Blázquez et al.: Stellar populations of early-type galaxies in different environments. I., Online Material p 3
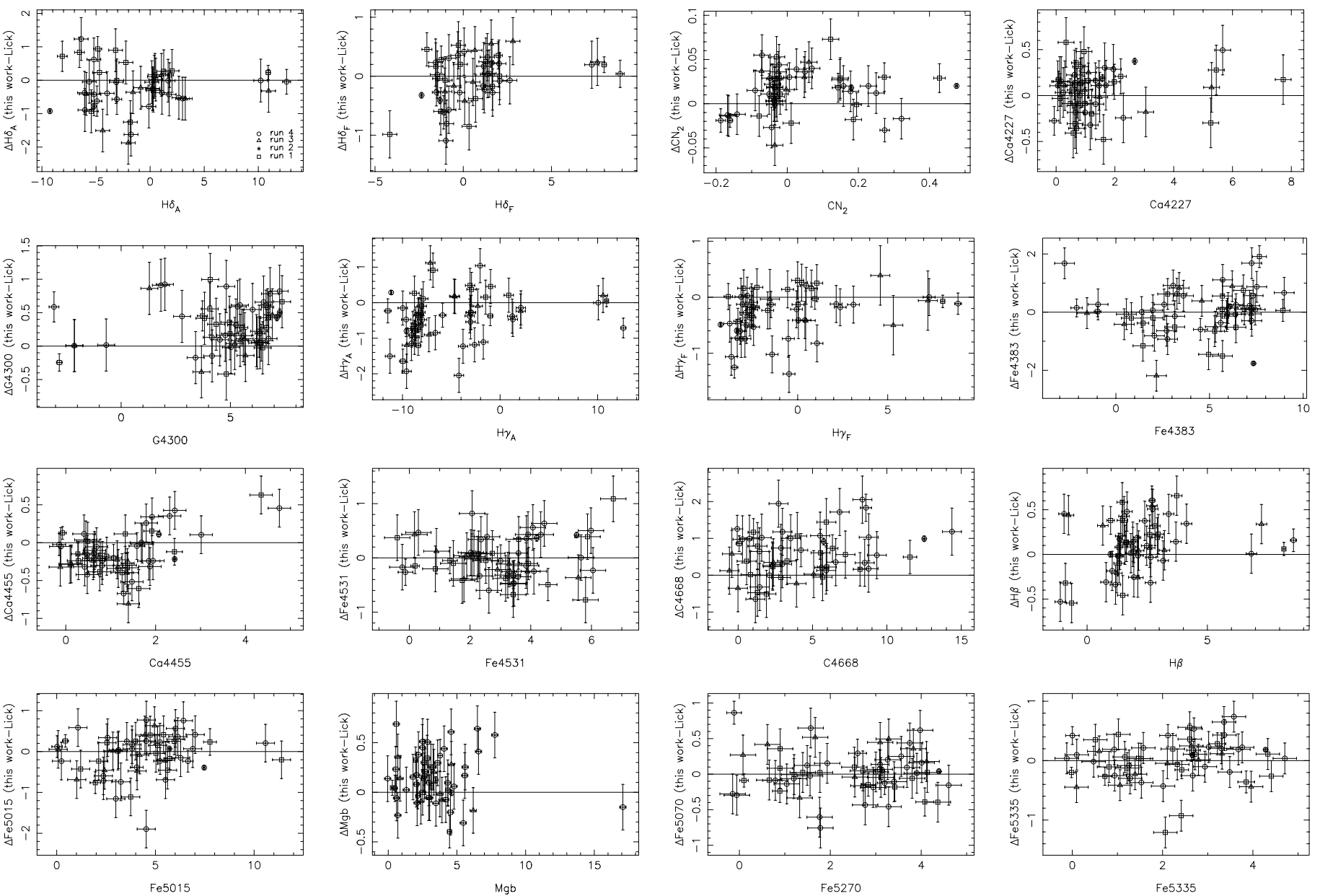

Fig. A.1. Differences between the indices measured in the Lick stars and our own measurements as a function of the latter. Different symbols represent the data obtained in the 4 observing runs; run1: squares; run2: stars; run3: triangles, and run4: circles. 


\section{Appendix B: Comparison of stars in different runs}

Figure B.1 shows the comparison of the indices measured in stars observed in Run 4 and other runs. Table B.1 indicates the mean offset, rms dispersion, and rms dispersion expected by errors. The last column of the table shows the $z$ parameter of the comparison. A $z$-value higher than 1.96 indicates that the offset is significant, with a significance level lower than 0.05. Fe5270 and Fe5335 could not be measured in Run 2 and therefore are not shown in the table.
Table B.1. Mean offset, rms $(\sigma)$, and rms expected by the errors $(\sigma(\exp ))$ in the comparison between stars observed in Run 4 and in the other runs. The last column shows the $z$ parameter, which indicates the significance of the mean offset. A $z$-value higher than 1.96 indicates that the offsets are significant with a significance level lower than 0.05 .

\begin{tabular}{lrrrr}
\hline \hline Index & Offset & $\sigma$ & $\sigma(\exp )$ & $z$ \\
\hline $\mathrm{H} \delta_{A}$ & -0.141 & 0.249 & 0.117 & 1.18 \\
$\mathrm{H} \delta_{F}$ & -0.021 & 0.135 & 0.092 & 0.38 \\
$\mathrm{CN}_{2}$ & -0.004 & 0.016 & 0.006 & 0.56 \\
$\mathrm{Ca} 4227$ & 0.095 & 0.279 & 0.045 & 0.78 \\
$\mathrm{G} 4300$ & -0.017 & 0.524 & 0.087 & 0.08 \\
$\mathrm{H} \gamma_{A}$ & -0.235 & 0.680 & 0.151 & 0.79 \\
$\mathrm{H} \gamma_{F}$ & 0.017 & 0.282 & 0.107 & 0.15 \\
$\mathrm{Fe} 4383$ & 0.129 & 0.362 & 0.201 & 0.81 \\
$\mathrm{Ca} 4455$ & 0.219 & 0.329 & 0.066 & 1.32 \\
$\mathrm{Fe} 4531$ & 0.099 & 0.146 & 0.097 & 1.34 \\
$\mathrm{C} 4668$ & -0.010 & 0.200 & 0.310 & 0.12 \\
$\mathrm{H} \beta$ & -0.045 & 0.091 & 0.063 & 1.06 \\
$\mathrm{Fe} 5015$ & 0.004 & 0.137 & 0.149 & 0.07 \\
$\mathrm{Mgb}$ & 0.077 & 0.079 & 0.067 & 1.64 \\
\hline
\end{tabular}


P. Sánchez-Blázquez et al.: Stellar populations of early-type galaxies in different environments. I., Online Material p 5

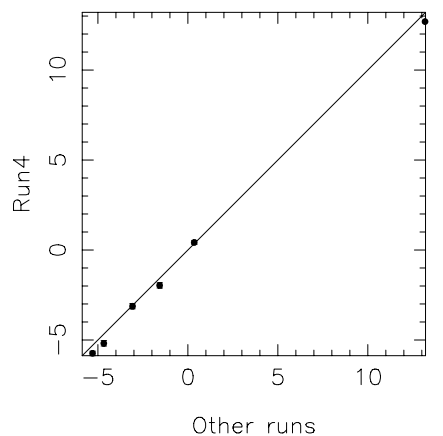

$\operatorname{Ca} 4227$

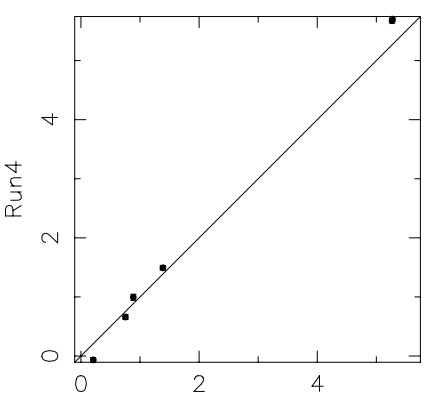

Other runs

Fe4383

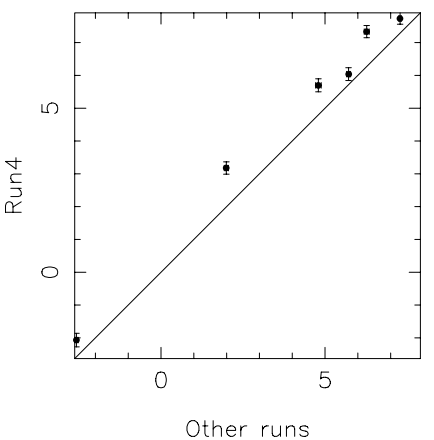

$H \beta$

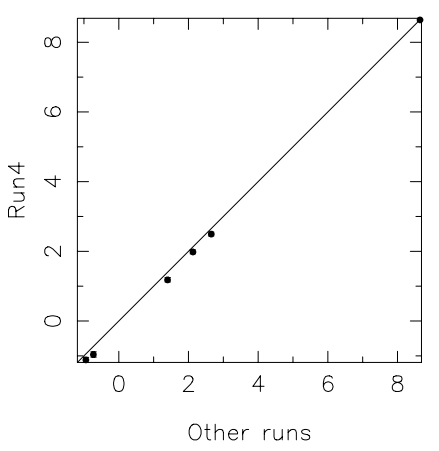

H $\delta F$

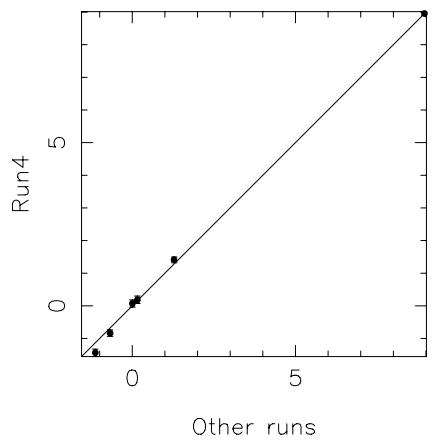

64300

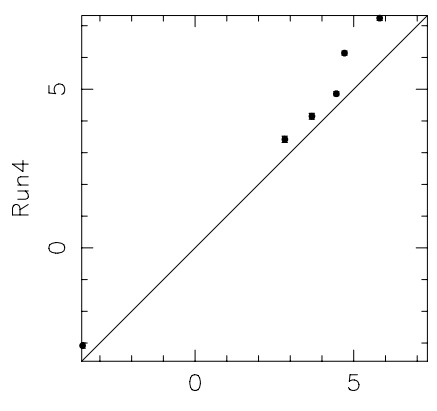

Other runs

Ca4455

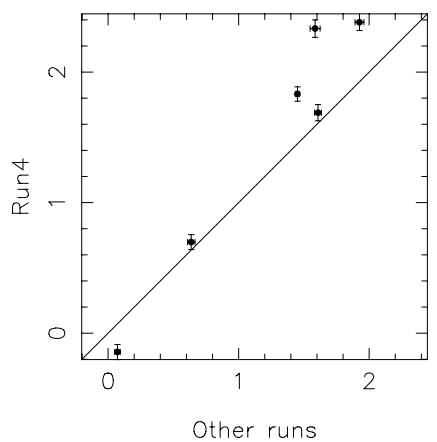

$\mathrm{CN}_{1}$

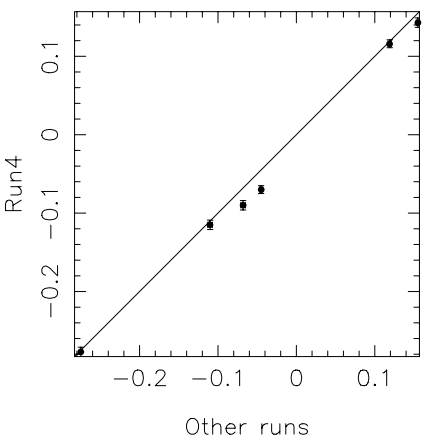

HyA

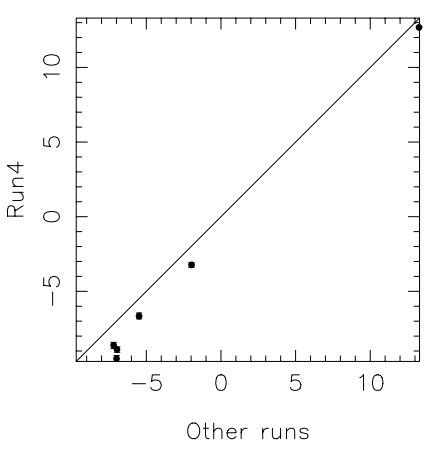

Fe4531

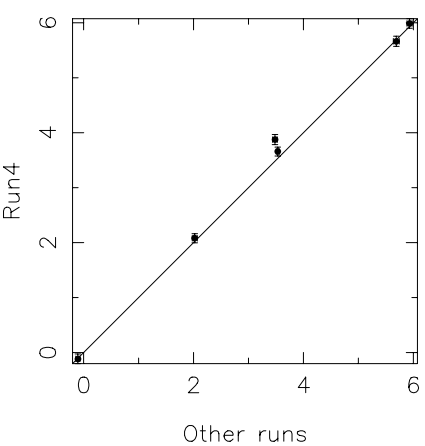

Fe5015

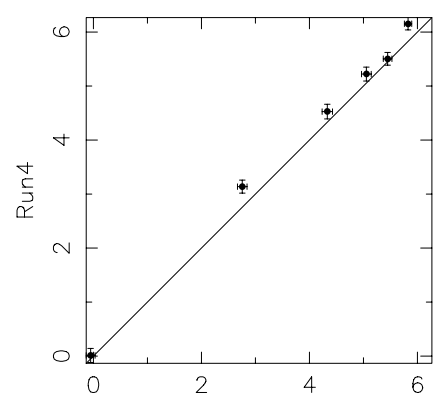

Other runs

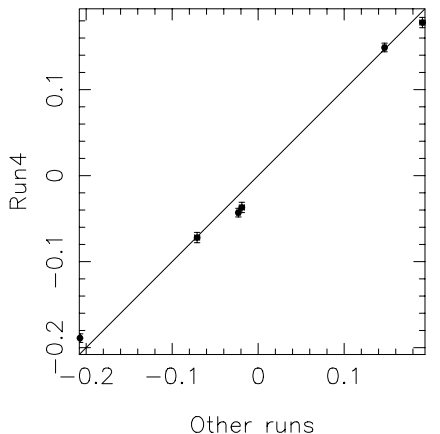

HyF

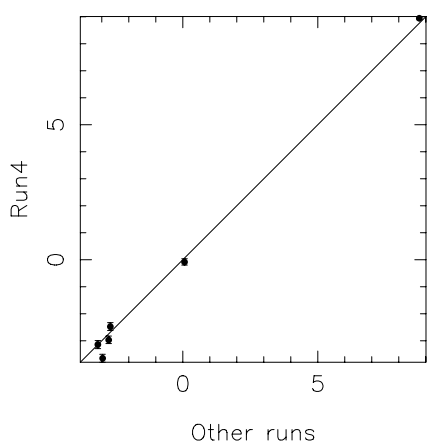

C4668

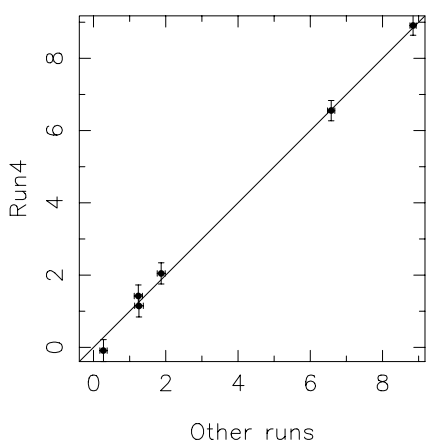

Mgo

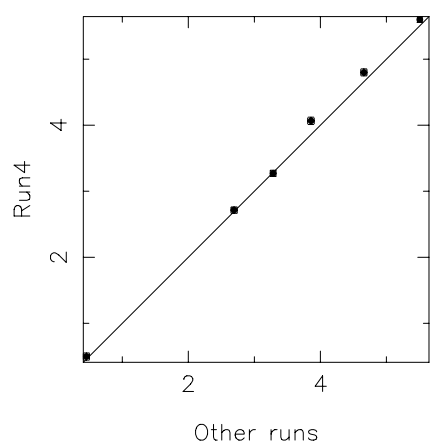

Fig. B.1. Comparison of the indices measured in the common stars between Run 4 and the other runs. 


\section{Appendix C: Velocity dispersion corrections for line-strengths}

The observed spectrum of a galaxy is the convolution of the integrated spectrum of its stellar population with the instrumental broadening and the distribution of line-of-sight velocities of the stars. The broadening of the spectra causes the indices to appear weaker than they intrinsically are. To compare line-strength indices among different galaxies, it is necessary to correct for the velocity dispersion and instrumental resolution broadening effects. This was done by using the optimal template obtained in the calculation of the velocity dispersion and radial velocity. This reference spectrum is the composition of the template stellar spectra that best matches the spectrum of each galaxy, corrected for differences in the intensities of the spectral lines. We used these composite templates instead of individual spectra because the variation of the indices with the broadening varies with their intensity (see Kuntschner 2000).

The templates were first broadened to the Lick resolution (which varies with wavelength) and then convolved with a Gaussian function of widths ranging from 0 to $450 \mathrm{~km} \mathrm{~s}^{-1}$ (in steps of $20 \mathrm{~km} \mathrm{~s}^{-1}$ ) to simulate the velocity dispersion broadening within a galaxy. Index strengths were measured for each spectrum and a correction factor was calculated as

$C(\sigma)=I(0) / I(\sigma)$,

where $I(0)$ is the index measured in the template spectrum, previously broadened to the instrumental resolution of Lick $\left(\sigma_{\text {lick }}\right)$, and $I(\sigma)$ the index measured in the same spectrum after being broadened by $\sigma$. Although a different polynomial was calculated for each galaxy spectrum, an average polynomial was applied to correct all the galaxies. This was done to avoid possible systematic effects due to a non-perfect match between the optimal template and the galaxy spectrum. Table C. 1 shows the final polynomial used to correct the indices for all the galaxies. The last column gives an estimate of the maximum error in each index due to this correction. This is calculated as the standard deviation of all the coefficients $C(\sigma)$ obtained with the different templates computed at $\sigma=400 \mathrm{~km} \mathrm{~s}^{-1}$. Note that all the corrections are multiplicative except for the $\mathrm{CN}_{1}, \mathrm{CN}_{2}, \mathrm{H} \delta_{A}$, and $\mathrm{H} \delta_{F}$ indices for which the corrections are additive $C(\sigma)=I(0)-I(\sigma)$.
Table C.1. Average polynomial coefficients for each line index correction function, where $\sigma *=\sigma_{\text {Lick }} / \sigma$. The last column shows the maximum error due to this correction for a galaxy of $\sigma=400 \mathrm{~km} \mathrm{~s}^{-1}$.

\begin{tabular}{|c|c|c|c|}
\hline Index & & $(\sigma)$ & Error \\
\hline $\mathrm{H} \delta_{A}$ & $0.0118-0.0005$ & $\times \sigma-1.5421 \times 10^{-6} \times \sigma^{2}$ & 0.175 \\
\hline $\mathrm{H} \delta_{F}$ & $-0.0013+2.4565 \times 10^{-5}$ & $5 \times \sigma+1.2798 \times 10^{-6} \times \sigma^{2}$ & 0.068 \\
\hline $\mathrm{CN}_{1}$ & $-0.0001+1.081 \times 10^{-5}$ & $\times \sigma+1.2259 \times 10^{-8} \times \sigma^{2}$ & 0.002 \\
\hline $\mathrm{CN}_{2}$ & $-0.0008+2.6876 \times 10^{-5}$ & $5 \times \sigma+6.1555 \times 10^{-8} \times \sigma^{2}$ & 0.006 \\
\hline Ca4227 & $0.7802-0.2809$ & $\times \sigma_{*}+0.5007$ & 0.059 \\
\hline G4300 & $0.8915+0.1000$ & $\times \sigma_{*}+0.0085$ & 0.014 \\
\hline $\mathrm{H} \gamma_{A}$ & $1.1184-0.2125$ & $\times \sigma_{*}+0.0940$ & 0.008 \\
\hline $\mathrm{H} \gamma_{F}$ & $0.8043+0.2565$ & $\times \sigma_{*}-0.0608$ & 0.035 \\
\hline $\mathrm{Fe} 4383$ & $0.8798-0.0095$ & $\times \sigma_{*}+0.1297$ & 0.025 \\
\hline $\mathrm{Ca} 4455$ & $0.6385+0.0962$ & $\times \sigma_{*}+0.2653$ & 0.034 \\
\hline $\mathrm{Fe} 4531$ & $0.9133-0.0123$ & $\times \sigma_{*}+0.0990$ & 0.009 \\
\hline C4668 & $1.0054-0.1268$ & $\times \sigma_{*}+0.1214$ & 0.013 \\
\hline $\mathrm{H} \beta$ & $0.9907+0.0056$ & $\times \sigma_{*}+0.0037$ & 0.012 \\
\hline Fe5015 & $0.7816+0.1791$ & $\times \sigma_{*}+0.0393$ & 0.023 \\
\hline $\mathrm{Mgb}$ & $0.9645-0.0749$ & $\times \sigma_{*}+0.1104$ & 0.020 \\
\hline Fe5270 & $0.8253+0.1228$ & $\times \sigma_{*}+0.0518$ & 0.004 \\
\hline Fe5335 & $0.8432-0.0814$ & $\times \sigma_{*}+0.2382$ & 0.051 \\
\hline
\end{tabular}




\section{Appendix D: Comparison with other authors}

Although we have checked the possible existence of offsets between different observing runs by comparing galaxies observed more than once, the number of available galaxies to make this comparison was admittedly sparse. Therefore, in this appendix, we repeat the comparison with other authors subdividing the galaxies in different observing runs. Table D.1 shows this comparison.

To check if the offsets with other authors were different in the different observing runs, we have performed a $t$-test comparing the offsets of Runs 2, 3, and 4 with the offsets obtained in Run 1. The results are shown in Table D.2. A value of $t$ larger than 1.96 indicates that the offset in the corresponding run is significatively different than the offset obtained in Run 1 (with a significance level lower than $\alpha=0.001$ ), and it would suggest the existence of offsets between runs. The only two cases in which that happens are in the comparison of $\mathrm{H} \gamma_{A}$ with the data of D05 (the offset in Run 2 is significatively larger than the one obtained in Run 1), and in the comparison of Fe4383 with the same reference, D05 (the offset in Run 3 is larger as well, but negative, than in the comparison with Run 1). In the first case, the number of galaxies in common between D05 and Run 2 is only 3 , which makes the result very uncertain. In the later case, we do not obtain significant differences in the offsets of Fe4383 between Run 3 and other runs when we compare them with other references (different than D05). We conclude, therefore, that the measurements obtained in different observing runs do not present any systematic difference.
Table D.1. Comparison of line strengths measured in this and other studies. Ref.: reference of the comparison work (see description in the text); $N$ : number of galaxies in common; $\Delta I$ : calculated offset between both studies (other studies minus this work); $\sigma$ : rms dispersion; $\sigma_{\text {exp }}$ : expected rms from the errors; $t$ : $t$-parameter of the comparison of means.

\begin{tabular}{|c|c|c|c|c|c|c|}
\hline$\overline{\overline{\text { Index }}}$ & Ref. & $\overline{\bar{N}}$ & $\overline{\overline{\Delta I}}$ & $\overline{\sigma \sigma}$ & $\overline{\sigma_{\exp }}$ & $t$ \\
\hline$\overline{\text { Run1 }}$ & & & & & & \\
\hline $\mathrm{H} \delta_{A}$ & $\mathrm{C} 03$ & 7 & $-0.095 \pm 0.129$ & 0.293 & 0.791 & 0.81 \\
\hline $\mathrm{H} \delta_{A}$ & D05 & 13 & $0.188 \pm 0.149$ & 0.459 & 0.531 & 1.36 \\
\hline $\mathrm{H} \gamma_{A}$ & $\mathrm{C} 03$ & 7 & $0.379 \pm 0.135$ & 0.305 & 0.710 & 1.96 \\
\hline $\mathrm{H} \gamma_{A}$ & D05 & 13 & $0.285 \pm 0.130$ & 0.426 & 0.532 & 1.98 \\
\hline $\mathrm{H} \delta_{F}$ & $\mathrm{C} 03$ & 7 & $-0.268 \pm 0.056$ & 0.126 & 0.586 & 2.25 \\
\hline $\mathrm{H} \delta_{F}$ & D05 & 13 & $-0.217 \pm 0.073$ & 0.185 & 0.399 & 2.68 \\
\hline $\mathrm{H} \gamma_{F}$ & $\mathrm{C} 03$ & 7 & $0.415 \pm 0.053$ & 0.142 & 0.518 & 2.34 \\
\hline $\mathrm{H} \gamma_{F}$ & D05 & 13 & $0.069 \pm 0.128$ & 0.441 & 0.478 & 0.55 \\
\hline $\mathrm{CN}_{2}$ & T98 & 19 & $-0.006 \pm 0.006$ & 0.023 & 0.190 & 1.05 \\
\hline $\mathrm{CN}_{2}$ & $\mathrm{C} 03$ & 7 & $-0.006 \pm 0.023$ & 0.051 & 0.128 & 0.30 \\
\hline $\mathrm{CN}_{2}$ & D05 & 13 & $-0.014 \pm 0.009$ & 0.028 & 0.093 & 1.62 \\
\hline Ca4227 & T98 & 19 & $-0.150 \pm 0.064$ & 0.289 & 0.735 & 2.26 \\
\hline Ca4227 & $\mathrm{C} 03$ & 7 & $-0.307 \pm 0.036$ & 0.082 & 0.469 & 2.38 \\
\hline Ca4227 & D05 & 13 & $-0.208 \pm 0.057$ & 0.141 & 0.301 & 2.90 \\
\hline G4300 & T98 & 19 & $-0.088 \pm 0.120$ & 0.460 & 0.763 & 0.80 \\
\hline G4300 & $\mathrm{C} 03$ & 7 & $-0.357 \pm 0.119$ & 0.269 & 0.607 & 2.01 \\
\hline G4300 & D05 & 13 & $-0.168 \pm 0.068$ & 0.222 & 0.474 & 2.14 \\
\hline Fe4383 & T98 & 19 & $-0.101 \pm 0.144$ & 0.543 & 1.039 & 0.79 \\
\hline $\mathrm{Fe} 4383$ & $\mathrm{C} 03$ & 7 & $0.066 \pm 0.099$ & 0.224 & 0.709 & 0.74 \\
\hline $\mathrm{Fe} 4383$ & D05 & 13 & $-0.641 \pm 0.120$ & 0.416 & 0.580 & 2.94 \\
\hline $\mathrm{Ca} 4455$ & T98 & 19 & $-0.083 \pm 0.062$ & 0.236 & 0.658 & 1.44 \\
\hline $\mathrm{Ca} 4455$ & D05 & 13 & $0.086 \pm 0.060$ & 0.200 & 0.387 & 1.41 \\
\hline $\mathrm{Fe} 4531$ & T98 & 19 & $-0.235 \pm 0.108$ & 0.417 & 0.837 & 2.13 \\
\hline Fe4531 & D05 & 13 & $-0.212 \pm 0.082$ & 0.261 & 0.482 & 2.24 \\
\hline C4668 & T98 & 19 & $-0.716 \pm 0.170$ & 0.664 & 1.104 & 3.15 \\
\hline C4668 & D05 & 13 & $-0.956 \pm 0.185$ & 0.572 & 0.675 & 3.00 \\
\hline $\mathrm{H} \beta$ & G93 & 17 & $-0.208 \pm 0.028$ & 0.113 & 0.326 & 3.54 \\
\hline $\mathrm{H} \beta$ & T98 & 19 & $0.129 \pm 0.065$ & 0.259 & 0.586 & 1.80 \\
\hline $\mathrm{H} \beta$ & K01 & 8 & $-0.050 \pm 0.043$ & 0.121 & 0.536 & 1.06 \\
\hline $\mathrm{H} \beta$ & $\mathrm{C} 03$ & 7 & $-0.016 \pm 0.049$ & 0.111 & 0.423 & 0.37 \\
\hline $\mathrm{H} \beta$ & D05 & 13 & $-0.179 \pm 0.100$ & 0.220 & 0.362 & 2.23 \\
\hline Fe5015 & G93 & 17 & $-0.281 \pm 0.151$ & 0.610 & 0.626 & 1.72 \\
\hline Fe5015 & T98 & 17 & $-0.251 \pm 0.149$ & 0.549 & 0.954 & 1.70 \\
\hline Fe5015 & $\mathrm{C} 03$ & 7 & $0.328 \pm 0.121$ & 0.281 & 0.674 & 1.92 \\
\hline Fe5015 & D05 & 13 & $-0.323 \pm 0.231$ & 0.744 & 0.577 & 1.43 \\
\hline $\mathrm{Mgb}$ & G93 & 17 & $-0.264 \pm 0.066$ & 0.267 & 0.375 & 2.86 \\
\hline $\mathrm{Mgb}$ & T98 & 19 & $-0.159 \pm 0.063$ & 0.262 & 0.677 & 2.16 \\
\hline Mgb & K01 & 8 & $-0.123 \pm 0.113$ & 0.316 & 0.579 & 1.02 \\
\hline Mgb & $\mathrm{C} 03$ & 7 & $0.301 \pm 0.088$ & 0.207 & 0.509 & 2.07 \\
\hline Mgb & D05 & 13 & $-0.011 \pm 0.077$ & 0.264 & 0.467 & 0.15 \\
\hline Fe5270 & T98 & 19 & $-0.088 \pm 0.060$ & 0.228 & 0.618 & 1.56 \\
\hline Fe5270 & $\mathrm{C} 03$ & 7 & $-0.188 \pm 0.098$ & 0.221 & 0.469 & 1.66 \\
\hline Fe5270 & D05 & 13 & $-0.275 \pm 0.057$ & 0.180 & 0.411 & 2.93 \\
\hline Fe5335 & T98 & 19 & $-0.098 \pm 0.077$ & 0.287 & 0.720 & 1.41 \\
\hline Fe5335 & D05 & 13 & $-0.243 \pm 0.080$ & 0.248 & 0.446 & 2.47 \\
\hline
\end{tabular}


P. Sánchez-Blázquez et al.: Stellar populations of early-type galaxies in different environments. I., Online Material p 8

Table D.1. continued.

\begin{tabular}{|c|c|c|c|c|c|c|}
\hline$\overline{\text { Index }}$ & $\overline{\text { Ref. }}$ & $\overline{\bar{N}}$ & $\overline{\overline{\Delta I}}$ & $\bar{\sigma}$ & $\sigma_{\exp }$ & $\bar{t}$ \\
\hline$\overline{\text { Run2 }}$ & & & & & & \\
\hline $\mathrm{H} \delta_{A}$ & D05 & 3 & $-0.172 \pm 0.251$ & 0.346 & 0.576 & 0.73 \\
\hline $\mathrm{H} \delta_{F}$ & D05 & 3 & $-0.256 \pm 0.046$ & 0.056 & 0.313 & 1.39 \\
\hline $\mathrm{H} \gamma_{A}$ & D05 & 3 & $-0.044 \pm 0.305$ & 0.477 & 0.533 & 0.16 \\
\hline $\mathrm{H} \gamma_{F}$ & D05 & 3 & $-0.016 \pm 0.390$ & 0.634 & 0.452 & 0.04 \\
\hline $\mathrm{CN}_{2}$ & T98 & 5 & $-0.017 \pm 0.020$ & 0.035 & 0.190 & 0.99 \\
\hline $\mathrm{CN}_{2}$ & D05 & 3 & $0.008 \pm 0.017$ & 0.020 & 0.104 & 0.60 \\
\hline $\mathrm{Ca} 4227$ & T98 & 5 & $-0.265 \pm 0.073$ & 0.120 & 0.709 & 1.85 \\
\hline $\mathrm{Ca} 4227$ & D05 & 3 & $-0.194 \pm 0.069$ & 0.088 & 0.215 & 1.33 \\
\hline G4300 & T98 & 5 & $-0.103 \pm 0.452$ & 0.767 & 0.769 & 0.30 \\
\hline G4300 & D05 & 3 & $-0.151 \pm 0.080$ & 0.134 & 0.479 & 1.14 \\
\hline $\mathrm{Fe} 4383$ & T98 & 5 & $-0.118 \pm 0.238$ & 0.408 & 1.039 & 0.62 \\
\hline $\mathrm{Fe} 4383$ & D05 & 3 & $-0.348 \pm 0.351$ & 0.549 & 0.610 & 0.87 \\
\hline Ca4455 & T98 & 5 & $-0.107 \pm 0.172$ & 0.279 & 0.635 & 0.99 \\
\hline Ca4455 & D05 & 3 & $0.158 \pm 0.151$ & 0.216 & 0.401 & 0.95 \\
\hline $\mathrm{Fe} 4531$ & T98 & 5 & $-0.263 \pm 0.180$ & 0.296 & 0.823 & 1.41 \\
\hline $\mathrm{Fe} 4531$ & D05 & 3 & $-0.035 \pm 0.236$ & 0.337 & 0.446 & 0.18 \\
\hline C4668 & T98 & 5 & $-0.632 \pm 0.521$ & 0.878 & 1.063 & 1.25 \\
\hline C4668 & D05 & 3 & $-0.798 \pm 0.385$ & 0.641 & 0.739 & 1.18 \\
\hline $\mathrm{H} \beta$ & G93 & 5 & $-0.314 \pm 0.117$ & 0.231 & 0.329 & 1.46 \\
\hline $\mathrm{H} \beta$ & T98 & 5 & $0.120 \pm 0.121$ & 0.204 & 0.575 & 1.10 \\
\hline $\mathrm{H} \beta$ & D05 & 3 & $-0.026 \pm 0.184$ & 0.231 & 0.247 & 0.19 \\
\hline $\mathrm{Fe} 5015$ & G93 & 4 & $-0.558 \pm 0.281$ & 0.483 & 0.614 & 1.15 \\
\hline $\mathrm{Fe} 5015$ & T98 & 3 & $-0.158 \pm 0.165$ & 0.235 & 0.807 & 0.90 \\
\hline $\mathrm{Fe} 5015$ & D05 & 3 & $-0.818 \pm 0.575$ & 0.945 & 0.736 & 1.03 \\
\hline Mgb & G93 & 5 & $-0.406 \pm 0.288$ & 0.572 & 0.374 & 1.10 \\
\hline Mgb & T98 & 5 & $-0.203 \pm 0.342$ & 0.607 & 0.675 & 0.70 \\
\hline Mgb & D05 & 3 & $0.144 \pm 0.018$ & 0.031 & 0.412 & 1.39 \\
\hline $\mathrm{Fe} 5270$ & T98 & 5 & $-0.031 \pm 0.134$ & 0.217 & 0.595 & 0.31 \\
\hline $\mathrm{Fe} 5270$ & D05 & 3 & $-0.247 \pm 0.164$ & 0.258 & 0.411 & 1.08 \\
\hline $\mathrm{Fe} 5335$ & T98 & 5 & $0.094 \pm 0.126$ & 0.203 & 0.680 & 1.02 \\
\hline $\mathrm{Fe} 5335$ & D05 & 3 & $-0.044 \pm 0.360$ & 0.545 & 0.464 & 0.14 \\
\hline
\end{tabular}

Table D.1. continued.

\begin{tabular}{|c|c|c|c|c|c|c|}
\hline Index & Ref. & $\overline{\bar{N}}$ & $\overline{\overline{\Delta I}}$ & $\sigma$ & $\sigma_{\exp }$ & $t$ \\
\hline \multicolumn{7}{|l|}{$\overline{\text { Run3 }}$} \\
\hline $\mathrm{H} \delta_{A}$ & $\mathrm{C} 03$ & 9 & $-0.098 \pm 0.132$ & 0 & 33 & 0.8 \\
\hline $\mathrm{H} \delta_{A}$ & D05 & 12 & & & & 1.78 \\
\hline $\mathrm{H} \gamma_{A}$ & $\mathrm{C} 03$ & 9 & $.453 \pm 0.080$ & & & 2.61 \\
\hline$\gamma_{A}$ & 05 & 12 & $.567 \pm 0.1$ & & 569 & 8 \\
\hline$\delta_{F}$ & 03 & 9 & $-0.243 \pm 0.050$ & 25 & 475 & 2.5 \\
\hline$\delta_{F}$ & D05 & 12 & $-0.080 \pm 0$ & 81 & 500 & 13 \\
\hline$\gamma_{F}$ & $\mathrm{C} 03$ & 9 & $0.422 \pm 0.064$ & 0.476 & .421 & 2.6 \\
\hline$\gamma_{F}$ & D05 & 12 & $0.321 \pm 0.061$ & 0.182 & 0.449 & 2.9 \\
\hline $\mathrm{N}_{2}$ & 98 & 31 & $-0.017 \pm 0.007$ & 0.037 & .203 & 2.3 \\
\hline $\mathrm{N}_{2}$ & 03 & J & $-0.013 \pm$ & & .118 & 1.6 \\
\hline $\mathrm{N}_{2}$ & 05 & 12 & $-0.036 \pm 0$ & & .094 & $2.4 C$ \\
\hline$a 422$ & T98 & 31 & $-0.198 \pm 0$ & & 787 & 2.57 \\
\hline 47 & $\mathrm{C} 03$ & 9 & -0 & & & 2.76 \\
\hline 4 & D05 & 12 & -0 . & & & 2.73 \\
\hline 128 & T98 & 31 & & & & 2.63 \\
\hline & & 9 & & & & .0 \\
\hline & 05 & 12 & & & & .0 \\
\hline 43 & 98 & 31 & -0 & & & .4 \\
\hline 243 & 33 & 9 & -0 . & & & 1.8 \\
\hline 243 & D05 & 12 & $-1 .($ & & & 3.1 \\
\hline 55 & 8 & 31 & -0.2 & & 7 & 3.1 \\
\hline 5 & D05 & 12 & -0 & & & 2.2 \\
\hline & & 31 & -0 . & & & 1.9 \\
\hline & - & 12 & -0 . & & & 3.1 \\
\hline & & 31 & -0 & & & 3.0 \\
\hline & & 12 & & & & 2.80 \\
\hline$\beta$ & G93 & 17 & & & & 1.45 \\
\hline$\beta$ & 98 & 31 & & & & 2.57 \\
\hline$B$ & & 17 & & & & 0.46 \\
\hline$\beta$ & & 9 & & & & 1.3 \\
\hline & & 12 & & & & 0.2 \\
\hline & J & 17 & & & & 2.8 \\
\hline & 98 & 31 & -0 & & & 2.3 \\
\hline & 03 & 9 & -0.2 & 0.237 & 0.530 & $2.1^{\prime}$ \\
\hline 50 & D05 & 12 & $-0.543 \pm 0.200$ & 0.632 & 0.601 & 2.2 \\
\hline \multicolumn{7}{|c|}{ 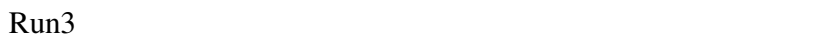 } \\
\hline & & 17 & & & & 2.2 \\
\hline & & 31 & -0 & & & 3.33 \\
\hline & 01 & 17 & -0 . & 38 & & 1.23 \\
\hline & $\mathrm{C} 03$ & 9 & & & & 0.50 \\
\hline & D05 & 12 & 05 & & 58 & 2.44 \\
\hline 5270 & T98 & 30 & 38 & & 693 & 1.32 \\
\hline & $\mathrm{C} 03$ & 9 & & & & 1.14 \\
\hline & D0 & 12 & 1 & & & 2.25 \\
\hline & 13 & 30 & & & & 1.52 \\
\hline $50 \pi+4$ & D05 & 12 & $-0.263 \pm 0.060$ & 0.176 & 0.448 & 2.7 \\
\hline
\end{tabular}


P. Sánchez-Blázquez et al.: Stellar populations of early-type galaxies in different environments. I., Online Material p 9

Table D.1. continued.

\begin{tabular}{lcccccc}
\hline \hline Index & Ref. & $N$ & \multicolumn{1}{c}{$\Delta I$} & $\sigma$ & $\sigma_{\exp }$ & $t$ \\
\hline Run 4 & & & & & & \\
CN $_{2}$ & T98 & 7 & $-0.010 \pm 0.014$ & 0.036 & 0.216 & 0.80 \\
Ca4227 & T98 & 7 & $-0.049 \pm 0.093$ & 0.237 & 0.895 & 0.54 \\
G4300 & T98 & 7 & $-0.407 \pm 0.376$ & 0.954 & 0.914 & 1.03 \\
Fe4383 & T98 & 7 & $0.181 \pm 0.478$ & 0.478 & 1.232 & 0.93 \\
Ca4455 & T98 & 7 & $-0.058 \pm 0.182$ & 0.460 & 0.831 & 0.33 \\
Fe4531 & T98 & 7 & $-0.310 \pm 0.174$ & 0.441 & 1.028 & 1.48 \\
C4668 & T98 & 7 & $-0.659 \pm 0.327$ & 0.831 & 1.310 & 1.59 \\
C4668 & M02 & 11 & $-1.133 \pm 0.446$ & 1.806 & 1.131 & 2.08 \\
H $\beta$ & T98 & 7 & $0.270 \pm 0.099$ & 0.251 & 0.690 & 1.86 \\
H $\beta$ & M00 & 6 & $-0.428 \pm 0.154$ & 0.316 & 0.418 & 1.86 \\
H $\beta$ & K01 & 9 & $-0.105 \pm 0.046$ & 0.138 & 0.618 & 1.77 \\
Fe5015 & T98 & 7 & $-0.068 \pm 0.118$ & 0.301 & 1.190 & 0.58 \\
Fe5015 & M02 & 11 & $0.003 \pm 0.248$ & 0.664 & 1.031 & 0.01 \\
Mgb & T98 & 7 & $-0.493 \pm 0.257$ & 0.657 & 0.840 & 1.54 \\
Mgb & M00 & 6 & $-0.158 \pm 0.099$ & 0.220 & 0.491 & 1.39 \\
Mgb & K01 & 9 & $-0.112 \pm 0.049$ & 0.189 & 0.664 & 1.78 \\
Fe5270 & T98 & 7 & $0.237 \pm 0.080$ & 0.202 & 0.769 & 1.92 \\
Fe5270 & M00 & 6 & $0.212 \pm 0.070$ & 0.153 & 0.480 & 1.87 \\
Fe5270 & M02 & 11 & $0.107 \pm 0.067$ & 0.218 & 0.490 & 1.55 \\
Fe5335 & T98 & 7 & $0.311 \pm 0.211$ & 0.530 & 0.922 & 1.31 \\
Fe5335 & M00 & 6 & $0.111 \pm 0.182$ & 0.398 & 0.514 & 0.65 \\
Fe5335 & M02 & 11 & $0.088 \pm 0.110$ & 0.358 & 0.607 & 0.79 \\
\hline
\end{tabular}


P. Sánchez-Blázquez et al.: Stellar populations of early-type galaxies in different environments. I., Online Material p 10

Table D.2. $t$-parameter in the comparison of the offsets between different observing runs and other authors. A $t$-value higher than 1.96 indicates that the offsets are significant different, which would suggest the existence of systematic differences between observing runs.

\begin{tabular}{|c|c|c|c|c|c|}
\hline$\overline{\overline{\text { Indice }}}$ & $\overline{\overline{\text { Ref }}}$ & 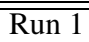 & 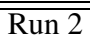 & 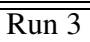 & $\overline{\overline{\text { Run4 }}}$ \\
\hline$\overline{\mathrm{H} \delta_{A}}$ & $(\mathrm{C} 03)$ & $\overline{0}$ & & 0.02 & \\
\hline $\mathrm{H} \delta_{A}$ & (D05) & 0 & 1.23 & 0.38 & \\
\hline $\mathrm{H} \delta_{F}$ & (D05) & 0 & 0.45 & 1.40 & \\
\hline $\mathrm{H} \delta_{F}$ & (C03) & 0 & 0.33 & & \\
\hline $\mathrm{H} \gamma_{A}$ & (C03) & 0 & & 0.47 & \\
\hline $\mathrm{H} \gamma_{A}$ & (D05) & 0 & 3.84 & 1.60 & \\
\hline $\mathrm{H} \gamma_{F}$ & (C03) & 0 & & 0.08 & \\
\hline $\mathrm{H} \gamma_{F}$ & (D05) & 0 & 0.21 & 1.77 & \\
\hline $\mathrm{CN}_{2}$ & (T98) & 0 & 0.52 & 1.19 & 0.26 \\
\hline $\mathrm{CN}_{2}$ & (D05) & 0 & 1.14 & 1.39 & \\
\hline $\mathrm{CN}_{2}$ & $(\mathrm{C} 03)$ & 0 & & 0.29 & \\
\hline $\mathrm{Ca} 4227$ & (T98) & 0 & 1.18 & 0.50 & 0.89 \\
\hline $\mathrm{Ca} 4227$ & (C03) & 0 & & 0.04 & \\
\hline $\mathrm{Ca} 4227$ & (D05) & 0 & 0.16 & 0.99 & \\
\hline G4300 & (T98) & 0 & 0.03 & 1.60 & 0.81 \\
\hline G4300 & $(\mathrm{C} 02)$ & 0 & & 0.14 & \\
\hline G4300 & (D05) & 0 & 0.16 & 1.29 & \\
\hline $\mathrm{Fe} 4383$ & (T98) & 0 & 0.06 & 0.30 & 0.56 \\
\hline $\mathrm{Fe} 4383$ & (C03) & 0 & & 1.65 & \\
\hline $\mathrm{Fe} 4383$ & (D05) & 0 & 0.78 & 2.44 & \\
\hline $\mathrm{Ca} 4455$ & (T98) & 0 & 0.13 & 1.53 & 0.13 \\
\hline $\mathrm{Ca} 4455$ & (D05) & 0 & 0.44 & 0.74 & \\
\hline $\mathrm{Fe} 4531$ & (T98) & 0 & 0.13 & 0.64 & 0.36 \\
\hline $\mathrm{Fe} 4531$ & (D05) & 0 & 0.71 & 1.91 & \\
\hline C4668 & (T98) & 0 & 0.15 & 0.45 & 0.15 \\
\hline C4668 & (D05) & 0 & 0.37 & 0.33 & \\
\hline $\mathrm{H} \beta$ & (T98) & 0 & 0.06 & 0.12 & 1.19 \\
\hline $\mathrm{H} \beta$ & (K01) & 0 & & 0.35 & 0.87 \\
\hline $\mathrm{H} \beta$ & (C03) & 0 & & 0.83 & \\
\hline $\mathrm{H} \beta$ & (D05) & 0 & 0.73 & 1.20 & \\
\hline Fe5015 & (G93) & 0 & 0.86 & 0.89 & \\
\hline Fe5015 & (T98) & 0 & 0.41 & 0.35 & 0.96 \\
\hline Fe5015 & (C03) & 0 & & 0.39 & \\
\hline Fe5015 & (D05) & 0 & 0.80 & 0.72 & \\
\hline
\end{tabular}

Table D.2. continued.

\begin{tabular}{lccccc}
\hline \hline Indice & Ref & Run 1 & Run 2 & Run 3 & Run4 \\
\hline Mgb & (G93) & 0 & 0.48 & 0.74 & \\
Mgb & (T98) & 0 & 0.13 & 2.07 & 1.26 \\
Mgb & (K01) & 0 & & 0.25 & 0.09 \\
Mgb & (C03) & 0 & & 0.96 & \\
Mgb & (D05) & 0 & 1.96 & 0.54 & \\
Fe5270 & (T98) & 0 & 0.38 & 1.92 & 3.25 \\
Fe5270 & (C03) & 0 & & 2.16 & \\
Fe5270 & (D05) & 0 & 0.16 & 1.72 & \\
Fe5335 & (T98) & 0 & 1.30 & 1.90 & 0.94 \\
Fe5335 & (D05) & 0 & 0.54 & 0.20 & \\
\hline
\end{tabular}


P. Sánchez-Blázquez et al.: Stellar populations of early-type galaxies in different environments. I., Online Material p 11

Table D.3. Fully corrected line-strength indices in the central $2^{\prime \prime} \times$ eqap aperture, where eqap represents $4 "$ at redshift $z=0.016$.

\begin{tabular}{|c|c|c|c|c|c|c|c|c|c|c|}
\hline $\begin{array}{c}\text { Galaxy } \\
\text { name }\end{array}$ & $\begin{array}{c}\begin{array}{c}\mathrm{D} 4000^{\prime} \\
(\mathrm{mag})\end{array}\end{array}$ & $\begin{array}{c}\mathrm{H} \delta_{A}^{\prime} \\
(\mathrm{mag})\end{array}$ & $\begin{array}{l}\mathrm{H} \delta_{F} 4 \\
(\mathrm{mag})\end{array}$ & $\begin{array}{l}\mathrm{CN}_{2} \\
(\mathrm{mag})\end{array}$ & $\begin{array}{c}\text { Ca4227' } \\
\text { (mag) }\end{array}$ & $\begin{array}{c}\begin{array}{c}\mathrm{G} 4300^{\prime} \\
(\mathrm{mag})\end{array}\end{array}$ & $\begin{array}{c}\mathrm{H} \gamma_{A}^{\prime} \\
(\mathrm{mag})\end{array}$ & $\begin{array}{c}\mathrm{H} \gamma_{F}^{\prime} \\
(\mathrm{mag})\end{array}$ & $\begin{array}{c}\begin{array}{c}\text { Fe4383' } \\
\text { (mag) }\end{array}\end{array}$ & $\begin{array}{l}\begin{array}{l}\text { Ca4455' } \\
\text { (mag) }\end{array} \\
\text { (a) }\end{array}$ \\
\hline \multirow[t]{2}{*}{ NGC 221 } & 0.784 & -0.026 & 0.040 & 0.039 & 0.078 & 0.158 & -0.097 & -0.007 & 0.109 & 0.078 \\
\hline & 0.013 & 0.001 & 0.001 & 0.014 & 0.001 & 0.001 & 0.000 & 0.004 & 0.001 & 0.002 \\
\hline \multirow[t]{2}{*}{ NGC 315} & 0.880 & -0.068 & -0.002 & 0.175 & 0.085 & 0.177 & -0.147 & -0.102 & 0.110 & 0.081 \\
\hline & 0.014 & 0.004 & 0.005 & 0.015 & 0.007 & 0.004 & 0.003 & 0.005 & 0.004 & 0.006 \\
\hline \multirow[t]{2}{*}{ NGC 507} & 0.843 & -0.073 & 0.001 & 0.145 & 0.100 & 0.160 & -0.122 & -0.056 & 0.107 & 0.081 \\
\hline & 0.084 & 0.009 & 0.012 & 0.018 & 0.014 & 0.010 & 0.007 & 0.010 & 0.009 & 0.011 \\
\hline \multirow[t]{2}{*}{ NGC 584} & 0.819 & -0.059 & 0.015 & 0.118 & 0.088 & 0.168 & -0.124 & -0.051 & 0.118 & 0.089 \\
\hline & 0.097 & 0.005 & 0.006 & 0.015 & 0.007 & 0.005 & 0.003 & 0.006 & 0.004 & 0.005 \\
\hline \multirow[t]{2}{*}{ NGC 636} & 0.812 & -0.061 & 0.013 & 0.124 & 0.100 & 0.175 & -0.132 & -0.061 & 0.116 & 0.078 \\
\hline & 0.098 & 0.004 & 0.005 & 0.015 & 0.006 & 0.004 & 0.003 & 0.005 & 0.004 & 0.004 \\
\hline \multirow[t]{2}{*}{ NGC 821} & 0.884 & -0.071 & 0.000 & 0.147 & 0.097 & 0.180 & -0.144 & -0.071 & 0.125 & 0.079 \\
\hline & 0.099 & 0.005 & 0.006 & 0.016 & 0.008 & 0.005 & 0.004 & 0.006 & 0.004 & 0.005 \\
\hline \multirow[t]{2}{*}{ NGC 1453} & 0.860 & -0.078 & -0.021 & 0.181 & 0.114 & 0.183 & -0.160 & -0.107 & 0.129 & 0.087 \\
\hline & 0.088 & 0.005 & 0.006 & 0.016 & 0.009 & 0.005 & 0.004 & 0.006 & 0.006 & 0.007 \\
\hline \multirow[t]{2}{*}{ NGC 1600} & 0.908 & -0.061 & 0.015 & 0.165 & 0.090 & 0.178 & -0.144 & -0.076 & 0.123 & 0.081 \\
\hline & 0.009 & 0.004 & 0.005 & 0.015 & 0.006 & 0.004 & 0.003 & 0.005 & 0.004 & 0.005 \\
\hline \multirow[t]{2}{*}{ NGC 1700} & 0.832 & -0.053 & 0.022 & 0.139 & 0.079 & 0.174 & -0.128 & -0.054 & 0.120 & 0.087 \\
\hline & 0.088 & 0.004 & 0.005 & 0.015 & 0.006 & 0.004 & 0.003 & 0.005 & 0.004 & 0.004 \\
\hline \multirow[t]{2}{*}{ NGC 2300} & 0.870 & -0.066 & -0.001 & 0.174 & 0.107 & 0.164 & -0.133 & -0.063 & 0.124 & 0.091 \\
\hline & 0.095 & 0.006 & 0.007 & 0.016 & 0.009 & 0.005 & 0.004 & 0.006 & 0.005 & 0.006 \\
\hline \multirow[t]{2}{*}{ NGC 2329} & 0.801 & -0.045 & 0.020 & 0.106 & 0.081 & 0.151 & -0.122 & -0.055 & 0.114 & 0.071 \\
\hline & 0.010 & 0.006 & 0.008 & 0.016 & 0.010 & 0.007 & 0.006 & 0.008 & 0.007 & 0.008 \\
\hline \multirow[t]{2}{*}{ NGC 2693} & 0.907 & -0.066 & -0.005 & 0.174 & 0.118 & 0.169 & -0.139 & -0.076 & 0.125 & 0.088 \\
\hline & 0.084 & 0.005 & 6 & 5 & 08 & .004 & 0.003 & 0.005 & 0.005 & 0.006 \\
\hline \multirow[t]{2}{*}{ NGC 2694} & 0.906 & -0.055 & 0.007 & 0.099 & 0.108 & 0.170 & -0.129 & -0.068 & 0.112 & 0.093 \\
\hline & 0.083 & 0.007 & 0.009 & .017 & 0.011 & 0.007 & 0.005 & 0.007 & 0.007 & 0.007 \\
\hline \multirow[t]{2}{*}{ NGC 2778} & 0.882 & -0.076 & -0.004 & 0.137 & 0.096 & 0.176 & -0.142 & -0.084 & 0.115 & 0.080 \\
\hline & 0.010 & 0.002 & 0.003 & 0.015 & 0.004 & 0.002 & 0.002 & 0.004 & 0.003 & 0.003 \\
\hline NGC 2832 & 0.907 & -0.073 & -0.013 & 0.163 & 0.097 & 0.165 & -0.152 & -0.097 & 0.125 & 0.082 \\
\hline & 0.009 & 0.003 & 0.004 & 0.015 & 0.006 & 0.004 & 0.003 & 0.005 & 0.004 & 0.005 \\
\hline NGC 3115 & 0.886 & -0.068 & 0.005 & 0.129 & 0.114 & 0.173 & -0.133 & -0.066 & 0.120 & 0.080 \\
\hline & 0.010 & 0.001 & 0.002 & 0.014 & 0.002 & 0.001 & 0.001 & 0.004 & 0.002 & 0.002 \\
\hline NGC 3377 & 0.778 & -0.037 & 0.025 & 0.092 & 0.078 & 0.155 & -0.108 & -0.026 & 0.108 & 0.073 \\
\hline & 0.102 & 0.003 & 0.004 & 0.015 & 0.004 & 0.003 & 0.002 & 0.004 & 0.003 & 0.003 \\
\hline NGC 3379 & 0.899 & -0.073 & -0.002 & 0.157 & 0.091 & 0.182 & -0.140 & -0.077 & 0.114 & 0.079 \\
\hline & 0.010 & 0.001 & 0.001 & & 0.002 & 0.001 & 0.001 & 0.004 & 0.002 & 0.001 \\
\hline NGC 3605 & 0.838 & -0.036 & 0.036 & 0.046 & 0.088 & 0.179 & -0.110 & -0.029 & 0.103 & 0.067 \\
\hline & 0.011 & 0.004 & 05 & & 07 & 05 & 0.004 & 0.006 & 0.005 & 0.005 \\
\hline NGC 3608 & 0.834 & -0.066 & -0.001 & 0. & 0.084 & 0.171 & -0.134 & -0.067 & 0.114 & 0.083 \\
\hline & 0.098 & 0.004 & 0.005 & 5 & 0.006 & 0.004 & 0.003 & 0.005 & 0.004 & 0.004 \\
\hline NGC 3641 & 0.902 & -0.056 & 0.011 & 0.1 & 0.083 & 0.171 & -0.119 & -0.046 & 0.100 & 0.071 \\
\hline & 0.010 & 0.003 & 0.004 & 0.015 & 0.006 & 0.004 & 0.003 & 0.005 & 0.004 & 0.004 \\
\hline NGC 3665 & 0.761 & -0.041 & 0.027 & 0.099 & 0.086 & 0.151 & -0.116 & -0.058 & 0.111 & 0.083 \\
\hline & 0.094 & 0.005 & 0.006 & 0.016 & 0.007 & 0.005 & 0.004 & 0.006 & 0.005 & 0.005 \\
\hline NGC 3818 & 0.901 & -0.066 & 0.001 & 0.134 & 0.097 & 0.167 & -0.132 & -0.069 & 0.111 & 0.077 \\
\hline & 0.010 & 0.002 & 0.002 & 0.014 & 0.003 & 0.002 & 0.002 & 0.004 & 0.002 & 0.002 \\
\hline NGC 4261 & 0.888 & -0.073 & -0.005 & 0.163 & 0.095 & 0.153 & -0.144 & -0.087 & 0.116 & 0.083 \\
\hline & 0.010 & 0.002 & 0.002 & 0.014 & 0.003 & 0.002 & 0.001 & 0.004 & 0.002 & 0.002 \\
\hline NGC 4278 & 0.864 & -0.074 & -0.018 & 0.161 & 0.088 & 0.178 & -0.160 & -0.126 & 0.112 & 0.078 \\
\hline & 0.010 & 0.001 & 0.001 & 0.014 & 0.002 & 0.001 & 0.001 & 0.003 & 0.002 & 0.002 \\
\hline NGC 4374 & 0.838 & -0.072 & -0.002 & 0.150 & 0.094 & 0.175 & -0.143 & -0.080 & 0.119 & 0.083 \\
\hline & 0.098 & 0.003 & 0.004 & 0.015 & 0.004 & 0.003 & 0.002 & 0.004 & 0.003 & 0.003 \\
\hline NGC 4431 & 0.616 & -0.016 & 0.034 & 0.004 & 0.091 & 0.157 & -0.101 & -0.015 & 0.087 & 0.051 \\
\hline & 0.099 & 0.008 & 0.010 & 0.017 & 0.012 & 0.008 & 0.006 & 0.009 & 0.007 & 0.008 \\
\hline NGC 4486B & 0.908 & -0.067 & 0.003 & 0.169 & 0.098 & 0.179 & -0.142 & -0.083 & 0.115 & 0.079 \\
\hline & 0.099 & 0.005 & 0.006 & & 0.008 & 0.005 & 0.004 & 0.006 & 0.004 & 0.005 \\
\hline NGC 4489 & 0.885 & -0.027 & 0.038 & 0.042 & 0.070 & 0.153 & -0.094 & -0.002 & 0.106 & 0.078 \\
\hline & 0.096 & 0.006 & 0.008 & 0.016 & 0.010 & 0.006 & 0.005 & 0.007 & 0.006 & 0.006 \\
\hline
\end{tabular}


P. Sánchez-Blázquez et al.: Stellar populations of early-type galaxies in different environments. I., Online Material p 12

Table D.3. continued.

\begin{tabular}{|c|c|c|c|c|c|c|c|c|c|c|}
\hline $\begin{array}{c}\text { Galaxy } \\
\text { name }\end{array}$ & $\begin{array}{r}\begin{array}{c}\text { D4000' } \\
(\mathrm{mag})\end{array} \\
\end{array}$ & $\begin{array}{c}\mathrm{H} \delta_{A}^{\prime} \\
(\mathrm{mag})\end{array}$ & $\begin{array}{c}\mathrm{H} \delta_{F}^{\prime} \\
(\mathrm{mag})\end{array}$ & $\begin{array}{c}\mathrm{CN}_{2} \\
(\mathrm{mag})\end{array}$ & $\begin{array}{c}\text { Ca4227' }^{\prime} \\
\text { (mag) }\end{array}$ & $\begin{array}{l}\begin{array}{l}\text { G4300' } \\
(\mathrm{mag})\end{array} \\
\end{array}$ & $\begin{array}{c}\mathrm{H} \gamma_{A}^{\prime} \\
(\mathrm{mag})\end{array}$ & $\begin{array}{c}\mathrm{H} \gamma_{F}^{\prime} \\
(\mathrm{mag})\end{array}$ & $\begin{array}{c}\begin{array}{c}\text { Fe4383' } \\
\text { (mag) }\end{array} \\
\end{array}$ & $\begin{array}{l}\begin{array}{l}\text { Ca4455' } \\
\text { (mag) }\end{array} \\
\end{array}$ \\
\hline \multirow[t]{2}{*}{$\overline{N G C ~} 4552$} & 0.820 & -0.063 & 0.006 & 0.164 & 0.100 & 0.168 & -0.140 & -0.083 & 0.110 & 0.082 \\
\hline & 0.101 & 0.004 & 0.004 & 0.015 & 0.004 & 0.003 & 0.002 & 0.004 & 0.003 & 0.003 \\
\hline \multirow[t]{2}{*}{ NGC 4636} & 0.808 & -0.070 & -0.006 & 0.161 & 0.097 & 0.171 & -0.145 & -0.086 & 0.115 & 0.080 \\
\hline & 0.099 & 0.006 & 0.007 & 0.016 & 0.008 & 0.005 & 0.004 & 0.006 & 0.005 & 0.005 \\
\hline \multirow[t]{2}{*}{ NGC 4649} & 0.855 & -0.085 & -0.011 & 0.230 & 0.120 & 0.169 & -0.141 & -0.078 & 0.121 & 0.090 \\
\hline & 0.097 & 0.005 & 0.005 & 0.015 & 0.008 & 0.004 & 0.003 & 0.005 & 0.004 & 0.006 \\
\hline \multirow[t]{2}{*}{ NGC 4365} & 0.924 & -0.073 & 0.002 & 0.168 & 0.105 & 0.188 & -0.134 & -0.052 & 0.120 & 0.077 \\
\hline & 0.010 & 0.002 & 0.002 & 0.014 & 0.003 & 0.002 & 0.001 & 0.004 & 0.002 & 0.002 \\
\hline \multirow{2}{*}{ NGC 4692} & 0.903 & -0.069 & 0.005 & 0.143 & 0.104 & 0.186 & -0.146 & -0.085 & 0.126 & 0.084 \\
\hline & 0.014 & 0.007 & 0.006 & 0.021 & 0.006 & 0.005 & 0.005 & 0.006 & 0.004 & 0.005 \\
\hline \multirow[t]{2}{*}{ NGC 4415} & 0.708 & -0.021 & 0.043 & 0.004 & 0.075 & 0.163 & -0.084 & 0.014 & 0.084 & 0.049 \\
\hline & 0.010 & 0.003 & 0.004 & 0.015 & 0.006 & 0.004 & 0.003 & 0.005 & 0.004 & 0.004 \\
\hline \multirow{2}{*}{ NGC 4464} & 0.857 & -0.061 & 0.007 & 0.103 & 0.078 & 0.175 & -0.128 & -0.048 & 0.104 & 0.064 \\
\hline & 0.010 & 0.002 & 0.002 & 0.014 & 0.003 & 0.002 & 0.001 & 0.004 & 0.002 & 0.002 \\
\hline \multirow[t]{2}{*}{ NGC 4467} & 0.850 & -0.067 & 0.007 & 0.112 & 0.091 & .170 & -0.127 & -0.044 & 0.112 & 0.074 \\
\hline & 0.010 & 0.004 & 0.005 & 0.015 & 0.007 & .005 & 0.003 & 0.006 & 0.005 & 0.005 \\
\hline \multirow[t]{2}{*}{ NGC 4472} & 0.929 & -0.082 & -0.010 & 0.184 & 0.117 & 0.177 & -0.149 & -0.084 & 0.129 & 0.095 \\
\hline & 0.010 & 0.003 & 0.003 & 0.015 & 0.005 & 0.003 & 0.002 & 0.004 & 0.003 & 0.004 \\
\hline \multirow[t]{2}{*}{ NGC 4478} & 0.816 & -0.044 & 0.025 & 0.083 & 0.096 & 0.165 & -0.119 & -0.041 & 0.109 & 0.072 \\
\hline & 0.010 & 0.001 & 0.002 & 0.014 & 0.002 & 0.002 & 0.001 & 0.004 & 0.002 & 0.002 \\
\hline \multirow[t]{2}{*}{ NGC 4564} & 0.873 & -0.071 & 0.001 & 0.150 & 0.096 & 0.169 & -0.139 & -0.063 & 0.124 & 0.080 \\
\hline & 0.010 & 0.001 & 0.002 & 0.014 & 0.002 & 0.001 & 0.001 & 0.004 & 0.002 & 0.002 \\
\hline \multirow[t]{2}{*}{ NGC 4594} & 0.940 & -0.073 & -0.011 & 0.157 & 0.099 & 0.185 & -0.152 & -0.090 & 0.116 & 0.079 \\
\hline & 0.011 & 0.006 & 0.008 & 0.016 & 0.010 & 0.006 & 0.005 & 0.007 & 0.006 & 0.007 \\
\hline \multirow[t]{2}{*}{ NGC 4621} & 0.892 & -0.072 & -0.002 & 0.153 & 0.097 & 0.179 & -0.139 & -0.081 & 0.112 & 0.083 \\
\hline & 0.010 & 0.002 & 0.002 & 0.014 & 0.003 & 0.002 & 0.002 & 0.004 & 0.002 & 0.002 \\
\hline \multirow[t]{2}{*}{ NGC 4673} & 0.825 & -0.037 & 0.041 & 0.117 & 0.072 & 0.166 & -0.111 & -0.042 & 0.108 & 0.076 \\
\hline & 0.013 & 0.009 & 0.007 & .018 & 0.004 & .004 & 0.005 & 0.006 & 0.003 & 0.003 \\
\hline NGC 4697 & 0.899 & -0.072 & 0.000 & 129 & 0.096 & 174 & -0.141 & -0.068 & 0.120 & 0.077 \\
\hline & 0.010 & 0.001 & 0.002 & 0.014 & 0.002 & .002 & 0.001 & 0.004 & 0.002 & 0.002 \\
\hline NGC 4742 & 0.701 & 0.083 & 0.152 & -0.010 & 0.043 & .082 & 0.003 & 0.123 & 0.075 & 0.056 \\
\hline & 0.010 & 0.001 & 0.001 & 0.014 & 0.001 & 0.001 & 0.001 & 0.004 & 0.002 & 0.001 \\
\hline NGC 4839 & 0.873 & -0.044 & 0.032 & 0.133 & 0.146 & 0.174 & -0.143 & -0.081 & 0.139 & 0.124 \\
\hline & 0.015 & 0.011 & 0.011 & 0.021 & 0.015 & 0.009 & 0.007 & 0.009 & 0.009 & 0.012 \\
\hline NGC 4842A & 0.863 & -0.046 & 0.022 & 0.109 & 0.117 & 0.180 & -0.150 & -0.082 & 0.121 & 0.077 \\
\hline & 0.014 & 0.009 & 0.008 & 0.020 & 0.007 & 0.005 & 0.006 & 0.006 & 0.005 & 0.005 \\
\hline NGC 4842B & 0.842 & -0.037 & 0.030 & 0.081 & 0.115 & 0.177 & -0.141 & -0.075 & 0.122 & 0.071 \\
\hline & 0.014 & 0.010 & 0.009 & 0.019 & 0.009 & 0.006 & 0.006 & 0.007 & 0.006 & 0.006 \\
\hline NGC 4864 & 0.841 & -0.034 & 0.044 & 0.103 & 0.102 & 0.176 & -0.138 & -0.080 & 0.112 & 0.084 \\
\hline & 0.014 & 0.010 & 0.010 & 18 & 0.010 & .007 & 0.007 & 0.008 & 0.006 & 0.008 \\
\hline NGC 4865 & 0.829 & -0.041 & 0.019 & 0.107 & 0.097 & .159 & -0.110 & -0.046 & 0.109 & 0.076 \\
\hline & 0.013 & 0.004 & 6 & & 0.006 & 05 & 0.005 & 0.007 & 004 & 0.004 \\
\hline NGC 4867 & 0.768 & -0.038 & 1 & & 0.098 & 0.148 & -0.107 & -0.041 & 04 & 0.067 \\
\hline & 0.013 & 0.006 & 0.008 & 7 & 0.009 & 0.007 & 0.006 & 0.008 & 0.006 & 0.007 \\
\hline NGC 4874 & 0.887 & -0.050 & 0.016 & 2 & 0.094 & 0.196 & -0.155 & -0.094 & 0.126 & 0.084 \\
\hline & 0.014 & 0.010 & 0.009 & 19 & 0.010 & 0.006 & 0.006 & 0.007 & 0.006 & 0.008 \\
\hline NGC 4875 & 0.875 & -0.020 & 0.048 & 0.094 & 0.094 & 0.167 & -0.125 & -0.060 & 0.105 & 0.078 \\
\hline & 0.015 & 0.008 & 0.007 & 0.021 & 0.007 & 0.006 & 0.005 & 0.007 & 0.005 & 0.006 \\
\hline NGC 4889 & 0.905 & -0.078 & -0.019 & 0.158 & 0.107 & 0.170 & -0.151 & -0.093 & 0.114 & 0.073 \\
\hline & 0.010 & 0.007 & 0.008 & 7 & 0.013 & 0.008 & 0.006 & 0.008 & 0.008 & 0.011 \\
\hline NGC 4908 & 0.853 & -0.058 & 0.015 & 0.078 & 0.101 & 0.171 & -0.124 & -0.057 & 0.106 & 0.062 \\
\hline & 0.014 & 0.006 & 0.006 & 0.021 & 0.008 & 0.005 & 0.005 & 0.007 & 0.004 & 0.005 \\
\hline NGC 5638 & 0.870 & -0.066 & 0.003 & 0.128 & 0.093 & 0.172 & -0.138 & -0.071 & 0.112 & 0.078 \\
\hline & 0.010 & 0.002 & 0.002 & 0.014 & 0.003 & 0.002 & 0.002 & 0.004 & 0.002 & 0.002 \\
\hline NGC 5796 & 0.875 & -0.076 & 0.001 & 0.155 & 0.101 & 0.150 & -0.130 & -0.066 & 0.120 & 0.086 \\
\hline & 0.009 & 0.003 & 0.004 & 0.015 & 0.005 & 0.003 & 0.003 & 0.005 & 0.004 & 0.004 \\
\hline NGC 5812 & 0.898 & -0.073 & 0.004 & 0.166 & 0.093 & 0.190 & -0.140 & -0.065 & 0.121 & 0.082 \\
\hline & 0.009 & 0.002 & 0.002 & 0.014 & 0.003 & 0.002 & 0.001 & 0.004 & 0.002 & 0.002 \\
\hline
\end{tabular}


Table D.3. continued.

\begin{tabular}{|c|c|c|c|c|c|c|c|c|c|c|}
\hline $\begin{array}{c}\text { Galaxy } \\
\text { name }\end{array}$ & $\begin{array}{c}\begin{array}{c}\text { D4000' } \\
(\mathrm{mag})\end{array}\end{array}$ & $\begin{array}{c}\mathrm{H} \delta_{A}^{\prime} \\
(\mathrm{mag})\end{array}$ & $\begin{array}{c}\mathrm{H} \delta_{F}^{\prime} \\
(\mathrm{mag})\end{array}$ & $\begin{array}{c}\mathrm{CN}_{2} \\
(\mathrm{mag})\end{array}$ & $\begin{array}{c}\text { Ca4227' } \\
\text { (mag) }\end{array}$ & $\begin{array}{c}\begin{array}{c}\text { G4300' } \\
(\mathrm{mag})\end{array}\end{array}$ & $\begin{array}{c}\mathrm{H} \gamma_{A}^{\prime} \\
(\mathrm{mag})\end{array}$ & $\begin{array}{c}\mathrm{H} \gamma_{F}^{\prime} \\
(\mathrm{mag})\end{array}$ & $\begin{array}{c}\begin{array}{c}\text { Fe4383' } \\
(\mathrm{mag})\end{array} \\
\end{array}$ & $\begin{array}{c}\begin{array}{c}\text { Ca4455' } \\
(\mathrm{mag})\end{array} \\
\end{array}$ \\
\hline \multirow[t]{2}{*}{$\overline{\text { NGC } 5813}$} & 0.917 & -0.081 & -0.009 & 0.156 & 0.091 & 0.190 & -0.144 & -0.079 & 0.111 & 0.075 \\
\hline & 0.010 & 0.002 & 0.002 & 0.014 & 0.003 & 0.002 & 0.001 & 0.004 & 0.002 & 0.002 \\
\hline \multirow[t]{2}{*}{ NGC 5831} & 0.865 & -0.060 & 0.011 & 0.114 & 0.102 & 0.185 & -0.131 & -0.058 & 0.116 & 0.076 \\
\hline & 0.010 & 0.002 & 0.002 & 0.014 & 0.003 & 0.002 & 0.002 & 0.004 & 0.002 & 0.002 \\
\hline \multirow[t]{2}{*}{ NGC 5845} & 0.912 & -0.067 & 0.004 & 0.138 & 0.091 & 0.193 & -0.138 & -0.064 & 0.115 & 0.079 \\
\hline & 0.010 & 0.001 & 0.002 & 0.014 & 0.002 & 0.002 & 0.001 & 0.004 & 0.002 & 0.002 \\
\hline \multirow[t]{2}{*}{ NGC 5846} & 0.894 & -0.079 & -0.009 & 0.176 & 0.095 & 0.189 & -0.146 & -0.085 & 0.116 & 0.083 \\
\hline & 0.010 & 0.002 & 0.003 & 0.015 & 0.004 & 0.003 & 0.002 & 0.004 & 0.003 & 0.003 \\
\hline \multirow[t]{2}{*}{ NGC 5846A } & 0.858 & -0.067 & 0.000 & 0.133 & 0.083 & 0.172 & -0.128 & -0.068 & 0.101 & 0.069 \\
\hline & 0.010 & 0.003 & 0.003 & 0.015 & 0.004 & 0.003 & 0.002 & 0.004 & 0.003 & 0.003 \\
\hline \multirow[t]{2}{*}{ NGC 6127} & 0.886 & -0.058 & 0.007 & 0.144 & 0.107 & 0.170 & -0.144 & -0.083 & 0.124 & 0.079 \\
\hline & 0.009 & 0.002 & 0.003 & 0.015 & 0.004 & 0.003 & 0.002 & 0.004 & 0.003 & 0.003 \\
\hline \multirow[t]{2}{*}{ NGC 6166} & 0.801 & -0.066 & -0.006 & 0.145 & 0.140 & 0.164 & -0.156 & -0.117 & 0.091 & 0.072 \\
\hline & 0.014 & 0.017 & 0.020 & 0.025 & 0.037 & 0.020 & 0.013 & 0.018 & 0.020 & 0.028 \\
\hline \multirow[t]{2}{*}{ NGC 6411} & 0.917 & -0.058 & 0.010 & 0.124 & 0.099 & 0.174 & -0.135 & -0.064 & 0.125 & 0.085 \\
\hline & 0.014 & 0.004 & 0.005 & 0.015 & 0.006 & 0.004 & 0.003 & 0.005 & 0.004 & 0.004 \\
\hline \multirow[t]{2}{*}{ NGC 6482} & 0.886 & -0.072 & -0.013 & 0.174 & 0.117 & 0.158 & -0.139 & -0.096 & 0.113 & 0.076 \\
\hline & 0.012 & 0.005 & 0.007 & 0.018 & 0.006 & 0.004 & 0.007 & 0.008 & 0.005 & 0.004 \\
\hline \multirow[t]{2}{*}{ NGC 6577} & 0.841 & -0.064 & -0.003 & 0.139 & 0.111 & 0.171 & -0.126 & -0.073 & 0.113 & 0.078 \\
\hline & 0.013 & 0.006 & 0.005 & 0.016 & 0.006 & 0.006 & 0.004 & 0.006 & 0.004 & 0.005 \\
\hline \multirow[t]{2}{*}{ NGC 6702} & 0.888 & -0.027 & 0.042 & 0.077 & 0.079 & 0.160 & -0.114 & -0.036 & 0.120 & 0.077 \\
\hline & 0.009 & 0.003 & 0.004 & 0.015 & 0.005 & 0.003 & 0.003 & 0.005 & 0.003 & 0.004 \\
\hline \multirow[t]{2}{*}{ NGC 6703} & 0.880 & -0.064 & 0.003 & 0.119 & 0.081 & 0.166 & -0.143 & -0.074 & 0.120 & 0.079 \\
\hline & 0.010 & 0.002 & 0.002 & 0.014 & 0.003 & 0.002 & 0.002 & 0.004 & 0.002 & 0.002 \\
\hline \multirow[t]{2}{*}{ NGC 7052} & 0.847 & -0.060 & -0.014 & 0.161 & 0.080 & 0.161 & -0.140 & -0.094 & 0.104 & 0.076 \\
\hline & 0.014 & 0.005 & 0.006 & 0.015 & 0.008 & 0.005 & 0.004 & 0.006 & 0.005 & 0.006 \\
\hline \multirow[t]{2}{*}{ IC 767} & 0.546 & 0.040 & 0.092 & -0.001 & 0.042 & 0.103 & -0.016 & 0.082 & 0.060 & 0.051 \\
\hline & 0.098 & 0.007 & 0.013 & 0.024 & 0.019 & 016 & 0.005 & 0.009 & 0.011 & 0.023 \\
\hline IC 794 & 0.761 & -0.049 & 0.028 & 0.025 & 0.093 & 0.140 & -0.101 & -0.013 & 0.104 & 0.066 \\
\hline & 0.010 & 0.006 & 0.008 & 0.016 & 0.011 & .007 & 0.005 & 0.008 & 0.007 & 0.008 \\
\hline IC 832 & 0.699 & 0.020 & 0.070 & 0.045 & 0.060 & 0.123 & -0.078 & -0.008 & 0.092 & 0.059 \\
\hline & 0.014 & 0.009 & 0.008 & 0.019 & 0.007 & 0.005 & 0.006 & 0.007 & 0.004 & 0.005 \\
\hline IC 3957 & 1.042 & -0.063 & 0.013 & 0.121 & 0.097 & 0.176 & -0.136 & -0.073 & 0.119 & 0.066 \\
\hline & 0.013 & 0.009 & 0.008 & 0.017 & 0.007 & 0.006 & 0.006 & 0.007 & 0.005 & 0.005 \\
\hline IC 3959 & 0.911 & -0.050 & 0.022 & 0.136 & 0.095 & 0.171 & -0.123 & -0.061 & 0.111 & 0.077 \\
\hline & 0.014 & 0.009 & 0.007 & 0.018 & 0.005 & 0.004 & 0.005 & 0.006 & 0.004 & 0.004 \\
\hline IC 3963 & 0.811 & -0.041 & 0.033 & 0.066 & 0.084 & 0.174 & -0.124 & -0.047 & 0.116 & 0.069 \\
\hline & 0.014 & 0.010 & 0.008 & 0.019 & 0.008 & 0.006 & 0.006 & 0.007 & 0.005 & 0.006 \\
\hline IC 3973 & 0.852 & -0.039 & 0.027 & 0.125 & 0.086 & 0.160 & -0.105 & -0.036 & 0.119 & 0.074 \\
\hline & 0.012 & 0.005 & 0.006 & 0.017 & 0.005 & 0.006 & 0.005 & 0.006 & 0.004 & 0.004 \\
\hline IC 4026 & 1.112 & -0.016 & 0.047 & 0.061 & 0.098 & 0.155 & -0.111 & -0.039 & 0.108 & 0.076 \\
\hline & 0.015 & 0.010 & 0.011 & 22 & 0.012 & 009 & 0.007 & 0.009 & 0.007 & 0.008 \\
\hline IC 4042 & 1.107 & -0.034 & 0.017 & & 0.069 & 0.126 & -0.125 & -0.056 & 0.110 & 0.058 \\
\hline & 0.013 & 0.010 & 0.009 & 7 & 0.008 & 0.007 & 0.006 & 0.007 & 0.005 & 0.006 \\
\hline IC 4051 & 0.875 & -0.078 & -0.004 & 0.216 & 0.094 & 0.192 & -0.140 & -0.079 & 0.114 & 0.089 \\
\hline & 0.013 & 0.006 & 0.007 & 0.017 & 0.009 & 0.007 & 0.005 & 0.007 & 0.006 & 0.007 \\
\hline CGCG159-041 & 0.860 & -0.066 & 0.012 & 0.143 & 0.079 & 0.167 & -0.125 & -0.063 & 0.116 & 0.065 \\
\hline & 0.013 & 0.009 & 0.008 & 0.017 & 0.006 & 0.005 & 0.006 & 0.006 & 0.004 & 0.005 \\
\hline CGCG159-043 & 0.982 & -0.077 & 0.011 & 0.183 & 0.086 & 0.184 & -0.144 & -0.091 & 0.113 & 0.076 \\
\hline & 0.014 & 0.009 & 0.008 & 0.018 & 0.006 & 0.005 & 0.005 & 0.006 & 0.004 & 0.005 \\
\hline CGCG159-083 & 0.711 & -0.001 & 0.033 & 0.055 & 0.068 & 0.136 & -0.100 & -0.035 & 0.101 & 0.059 \\
\hline & 0.014 & 0.009 & 0.008 & 0.018 & 0.006 & 0.005 & 0.006 & 0.007 & 0.004 & 0.005 \\
\hline CGCG159-089 & 0.915 & -0.050 & 0.019 & 0.088 & 0.087 & 0.168 & -0.127 & -0.057 & 0.109 & 0.075 \\
\hline & 0.014 & 0.009 & 0.007 & 0.021 & 0.007 & 0.005 & 0.005 & 0.007 & 0.005 & 0.005 \\
\hline DRCG 27-032 & 0.829 & -0.032 & 0.015 & 0.019 & 0.126 & 0.149 & -0.112 & -0.049 & 0.104 & 0.086 \\
\hline & 0.016 & 0.014 & 0.016 & 0.025 & 0.022 & 0.015 & 0.011 & 0.014 & 0.013 & 0.015 \\
\hline DRCG 27-127 & 0.916 & -0.045 & 0.019 & 0.054 & 0.112 & 0.175 & -0.133 & -0.073 & 0.109 & 0.087 \\
\hline & 0.014 & 0.011 & 0.011 & 0.022 & 0.012 & 0.009 & 0.007 & 0.009 & 0.008 & 0.009 \\
\hline
\end{tabular}


P. Sánchez-Blázquez et al.: Stellar populations of early-type galaxies in different environments. I., Online Material p 14

Table D.3. continued.

\begin{tabular}{|c|c|c|c|c|c|c|c|c|c|c|}
\hline $\begin{array}{l}\text { Galaxy } \\
\text { name }\end{array}$ & $\begin{array}{c}\begin{array}{c}\text { D4000' } \\
(\mathrm{mag})\end{array}\end{array}$ & $\begin{array}{c}\mathrm{H} \delta_{A}^{\prime} \\
(\mathrm{mag})\end{array}$ & $\begin{array}{c}\mathrm{H} \delta_{F}^{\prime} \\
(\mathrm{mag})\end{array}$ & $\begin{array}{c}\mathrm{CN}_{2} \\
(\mathrm{mag})\end{array}$ & $\begin{array}{c}\text { Ca4227' } \\
\text { (mag) }\end{array}$ & 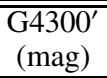 & $\begin{array}{c}\mathrm{H} \gamma_{A}^{\prime} \\
(\mathrm{mag})\end{array}$ & $\begin{array}{c}\mathrm{H} \gamma_{F}^{\prime} \\
(\mathrm{mag})\end{array}$ & $\begin{array}{c}\begin{array}{c}\text { Fe4383' } \\
(\mathrm{mag})\end{array}\end{array}$ & $\begin{array}{c}\text { Ca4455' } \\
\text { (mag) }\end{array}$ \\
\hline \multirow[t]{2}{*}{$\overline{\text { DRCG 27-128 }}$} & 0.893 & -0.036 & 0.022 & 0.016 & 0.104 & 0.172 & -0.122 & -0.045 & 0.113 & 0.066 \\
\hline & 0.014 & 0.008 & 0.008 & 0.022 & 0.010 & 0.007 & 0.006 & 0.008 & 0.006 & 0.007 \\
\hline \multirow[t]{2}{*}{ GMP 2585} & 0.611 & 0.009 & 0.054 & -0.038 & 0.062 & 0.112 & -0.055 & 0.033 & 0.069 & 0.060 \\
\hline & 0.015 & 0.013 & 0.014 & 0.022 & 0.017 & 0.012 & 0.010 & 0.013 & 0.011 & 0.013 \\
\hline \multirow[t]{2}{*}{ GMP 3121} & 0.728 & -0.012 & 0.029 & 0.038 & 0.099 & 0.166 & -0.119 & -0.031 & 0.122 & 0.080 \\
\hline & 0.016 & 0.014 & 0.016 & 0.024 & 0.019 & 0.014 & 0.010 & 0.014 & 0.012 & 0.014 \\
\hline \multirow[t]{2}{*}{ GMP 3131} & 0.675 & -0.014 & 0.060 & -0.036 & 0.075 & 0.136 & -0.070 & 0.003 & 0.071 & 0.040 \\
\hline & 0.016 & 0.014 & 0.016 & 0.024 & 0.020 & 0.015 & 0.012 & 0.015 & 0.013 & 0.015 \\
\hline \multirow[t]{2}{*}{ GMP 3196} & 0.658 & 0.014 & 0.048 & -0.027 & 0.088 & 0.151 & -0.097 & -0.015 & 0.099 & 0.049 \\
\hline & 0.014 & 0.011 & 0.011 & 0.020 & 0.012 & 0.009 & 0.008 & 0.010 & 0.008 & 0.009 \\
\hline \multirow[t]{2}{*}{$\mathrm{MCG}+5-31-63$} & 0.817 & -0.018 & 0.042 & 0.044 & 0.076 & 0.178 & -0.124 & -0.061 & 0.093 & 0.052 \\
\hline & 0.014 & 0.009 & 0.009 & 0.022 & 0.010 & 0.007 & 0.006 & 0.008 & 0.006 & 0.007 \\
\hline \multirow[t]{2}{*}{ PCG 126756} & 0.642 & 0.011 & 0.052 & -0.024 & 0.046 & 0.132 & -0.073 & 0.006 & 0.080 & 0.061 \\
\hline & 0.015 & 0.011 & 0.012 & 0.023 & 0.014 & 0.011 & 0.009 & 0.011 & 0.009 & 0.011 \\
\hline \multirow[t]{2}{*}{ PCG 126775} & 0.617 & 0.011 & 0.067 & 0.031 & 0.067 & 0.160 & -0.068 & 0.034 & 0.068 & 0.058 \\
\hline & 0.015 & 0.013 & 0.014 & 0.021 & 0.020 & 0.014 & 0.010 & 0.014 & 0.012 & 0.014 \\
\hline \multirow[t]{2}{*}{ RB 091} & 1.116 & -0.049 & 0.023 & 0.061 & 0.097 & 0.175 & -0.132 & -0.057 & 0.117 & 0.062 \\
\hline & 0.015 & 0.010 & 0.012 & 0.023 & 0.014 & 0.011 & 0.006 & 0.009 & 0.008 & 0.009 \\
\hline
\end{tabular}


P. Sánchez-Blázquez et al.: Stellar populations of early-type galaxies in different environments. I., Online Material p 15

Table D.3. continued.

\begin{tabular}{|c|c|c|c|c|c|c|c|c|c|c|}
\hline $\begin{array}{l}\text { Galaxy } \\
\text { name }\end{array}$ & $\begin{array}{c}\begin{array}{c}\text { Fe4531' } \\
\text { (mag) }\end{array} \\
\end{array}$ & $\begin{array}{c}\text { C4668' } \\
(\mathrm{mag})\end{array}$ & $\begin{array}{c}\mathrm{H} \beta^{\prime} \\
(\mathrm{mag})\end{array}$ & $\begin{array}{c}\begin{array}{c}\text { Fe5015' } \\
(\mathrm{mag})\end{array} \\
\end{array}$ & $\begin{array}{l}\mathrm{Mgb}^{\prime} \\
(\mathrm{mag})\end{array}$ & $\begin{array}{c}\begin{array}{c}\text { Fe5270' } \\
(\mathrm{mag})\end{array} \\
\end{array}$ & $\begin{array}{c}\begin{array}{c}\text { Fe5335' } \\
\text { (mag) }\end{array} \\
\end{array}$ & $\begin{array}{l}<\mathrm{Fe}>^{\prime} \\
(\mathrm{mag})\end{array}$ & $\begin{array}{c}\sigma \\
\left(\mathrm{km} \mathrm{s}^{-1}\right)\end{array}$ & $\begin{array}{c}E W([\mathrm{O} \text { III }]) \\
(\AA)\end{array}$ \\
\hline \multirow[t]{2}{*}{ NGC 221} & 0.083 & 0.062 & 0.087 & 0.078 & 0.099 & 0.083 & 0.072 & 0.078 & 82.5 & 0.000 \\
\hline & 0.001 & 0.003 & 0.001 & 0.002 & 0.008 & 0.002 & 0.000 & 0.001 & 1.0 & 0.000 \\
\hline \multirow[t]{2}{*}{ NGC 315} & 0.085 & 0.093 & 0.052 & 0.035 & 0.187 & 0.080 & 0.068 & 0.074 & 303.9 & -2.169 \\
\hline & 0.004 & 0.004 & 0.003 & 0.003 & 0.009 & 0.004 & 0.005 & 0.003 & 5.4 & 0.020 \\
\hline \multirow{2}{*}{ NGC 507} & 0.085 & 0.091 & 0.053 & 0.075 & 0.154 & 0.087 & 0.095 & 0.091 & 265.9 & 0.000 \\
\hline & 0.007 & 0.006 & 0.006 & 0.006 & 0.011 & 0.008 & 0.010 & 0.006 & 9.5 & 0.000 \\
\hline \multirow[t]{2}{*}{ NGC 584} & 0.085 & 0.094 & 0.066 & 0.080 & 0.143 & 0.083 & 0.079 & 0.081 & 224.4 & -0.154 \\
\hline & 0.003 & 0.006 & 0.004 & 0.004 & 0.009 & 0.004 & 0.005 & 0.003 & 4.3 & 0.015 \\
\hline \multirow[t]{2}{*}{ NGC 636} & 0.088 & 0.094 & 0.065 & 0.088 & 0.142 & 0.084 & 0.082 & 0.083 & 182.0 & 0.000 \\
\hline & 0.003 & 0.005 & 0.003 & 0.004 & 0.008 & 0.003 & 0.004 & 0.003 & 2.3 & 0.000 \\
\hline \multirow[t]{2}{*}{ NGC 821} & 0.096 & 0.100 & 0.068 & 0.086 & 0.162 & 0.083 & 0.077 & 0.080 & 212.4 & 0.000 \\
\hline & 0.004 & 0.006 & 0.004 & 0.004 & 0.009 & 0.004 & 0.005 & 0.003 & 4.0 & 0.000 \\
\hline \multirow[t]{2}{*}{ NGC 1453} & 0.093 & 0.108 & 0.050 & 0.077 & 0.193 & 0.093 & 0.086 & 0.090 & 336.6 & -1.159 \\
\hline & 0.004 & 0.005 & 0.004 & 0.004 & 0.010 & 0.005 & 0.007 & 0.005 & 7.9 & 0.049 \\
\hline \multirow{2}{*}{ NGC 1600} & 0.091 & 0.101 & 0.055 & 0.079 & 0.184 & 0.083 & 0.089 & 0.086 & 308.1 & 0.000 \\
\hline & 0.004 & 0.004 & 0.003 & 0.004 & 0.011 & 0.003 & 0.003 & 0.002 & 4.1 & 0.000 \\
\hline \multirow[t]{2}{*}{ NGC 1700} & 0.092 & 0.108 & 0.070 & 0.093 & 0.146 & 0.087 & 0.084 & 0.086 & 252.7 & 0.000 \\
\hline & 0.003 & 0.004 & 0.003 & 0.003 & 0.009 & 0.004 & 0.006 & 0.004 & 2.9 & 0.000 \\
\hline \multirow[t]{2}{*}{ NGC 2300} & 0.092 & 0.105 & 0.059 & 0.086 & 0.182 & 0.081 & 0.082 & 0.082 & 303.5 & 0.000 \\
\hline & 0.004 & 0.006 & 0.004 & 0.004 & 0.009 & 0.004 & 0.006 & 0.003 & 5.8 & 0.000 \\
\hline \multirow[t]{2}{*}{ NGC 2329} & 0.089 & 0.090 & 0.047 & 0.074 & 0.177 & 0.083 & 0.074 & 0.078 & 228.3 & -0.523 \\
\hline & 0.006 & 0.006 & 0.006 & 0.006 & 0.015 & 0.006 & 0.009 & 0.005 & 4.4 & 0.017 \\
\hline \multirow[t]{2}{*}{ NGC 2693} & 0.090 & 0.107 & 0.043 & 0.083 & 0.179 & 0.092 & 0.075 & 0.084 & 322.1 & 0.000 \\
\hline & 0.004 & 0.004 & 0.003 & 0.004 & 0.009 & 0.006 & 0.007 & 0.005 & 4.5 & 0.000 \\
\hline \multirow[t]{2}{*}{ NGC 2694} & 0.082 & 0.099 & 0.059 & 0.079 & 0.156 & 0.085 & 0.065 & 0.075 & 142.7 & 0.000 \\
\hline & 0.005 & 0.005 & 0.005 & 0.005 & 0.010 & 0.007 & 0.008 & 0.005 & 3.7 & 0.000 \\
\hline \multirow[t]{2}{*}{ NGC 2778} & 0.088 & 0.098 & 0.070 & 0.068 & 0.152 & 0.086 & 0.076 & 0.081 & 167.4 & -0.998 \\
\hline & 0.002 & 0.003 & 0.002 & 0.002 & 0.008 & 0.002 & 0.002 & 0.001 & 1.3 & 0.019 \\
\hline NGC 2832 & 0.091 & 0.109 & 0.052 & 0.092 & 0.187 & 0.094 & 0.083 & 0.088 & 322.1 & 0.000 \\
\hline & 0.003 & 0.004 & 0.003 & 0.003 & 0.015 & 0.010 & 0.014 & 0.009 & 2.3 & 0.000 \\
\hline NGC 3115 & 0.086 & 0.095 & 0.057 & 0.081 & 0.164 & 0.096 & 0.087 & 0.091 & 283.7 & 0.000 \\
\hline & 0.001 & 0.003 & 0.002 & 0.002 & 0.008 & 0.006 & 0.009 & 0.006 & 1.8 & 0.000 \\
\hline NGC 3377 & 0.079 & 0.074 & 0.074 & 0.072 & 0.135 & 0.071 & 0.062 & 0.067 & 142.0 & -0.178 \\
\hline & 0.002 & 0.006 & 0.003 & 0.003 & 0.008 & 0.002 & 0.003 & 0.002 & 1.7 & 0.013 \\
\hline NGC 3379 & 0.085 & 0.093 & 0.060 & 0.073 & 0.146 & 0.086 & 0.078 & 0.082 & 228.1 & -0.272 \\
\hline & 0.001 & 0.003 & 0.002 & 0.002 & 0.008 & 0.002 & 0.001 & 0.001 & 1.1 & 0.015 \\
\hline NGC 3605 & 0.080 & 0.071 & 0.081 & 0.072 & 0.116 & 0.089 & 0.078 & 0.083 & 91.0 & -0.192 \\
\hline & 0.004 & 0.004 & 0.004 & 0.004 & 0.009 & 0.002 & 0.002 & 0.001 & 1.5 & 0.015 \\
\hline NGC 3608 & 0.087 & 0.093 & 0.059 & 0.081 & 0.162 & 0.079 & 0.073 & 0.076 & 203.2 & -0.245 \\
\hline & 0.003 & 0.006 & 0.003 & 0.003 & 0.009 & 0.003 & 0.004 & 0.002 & 3.8 & 0.018 \\
\hline NGC 3641 & 0.083 & 0.076 & 0.067 & 0.067 & 0.138 & 0.092 & 0.067 & 0.080 & 163.4 & -0.451 \\
\hline & 0.003 & 0.004 & 0.003 & 0.003 & 0.009 & 0.004 & 0.005 & 0.003 & 1.7 & 0.019 \\
\hline NGC 3665 & 0.089 & 0.090 & 0.054 & 0.080 & 0.148 & 0.080 & 0.080 & 0.080 & 222.5 & -0.349 \\
\hline & 0.004 & 0.006 & 0.004 & 0.004 & 0.009 & 0.004 & 0.005 & 0.003 & 5.3 & 0.013 \\
\hline NGC 3818 & 0.084 & 0.093 & 0.065 & 0.071 & 0.141 & 0.086 & 0.081 & 0.084 & 187.5 & -0.529 \\
\hline & 0.002 & 0.003 & 0.002 & 0.002 & 0.008 & 0.003 & 0.002 & 0.002 & 1.3 & 0.017 \\
\hline NGC 4261 & 0.087 & 0.106 & 0.064 & 0.069 & 0.186 & 0.093 & 0.086 & 0.090 & 302.7 & -0.744 \\
\hline & 0.002 & 0.003 & 0.002 & 0.002 & 0.014 & 0.002 & 0.002 & 0.001 & 1.7 & 0.018 \\
\hline NGC 4278 & 0.084 & 0.083 & 0.049 & 0.039 & 0.185 & 0.078 & 0.075 & 0.076 & 277.3 & -2.778 \\
\hline & 0.001 & 0.003 & 0.002 & 0.002 & 0.008 & 0.002 & 0.001 & 0.001 & 1.8 & 0.020 \\
\hline NGC 4374 & 0.083 & 0.087 & 0.053 & 0.076 & 0.171 & 0.078 & 0.071 & 0.075 & 300.7 & -0.396 \\
\hline & 0.002 & 0.006 & 0.002 & 0.003 & 0.008 & 0.003 & 0.003 & 0.002 & 4.1 & 0.016 \\
\hline NGC 4431 & 0.066 & 0.050 & 0.065 & 0.068 & 0.103 & 0.059 & 0.055 & 0.057 & 60.6 & 0.000 \\
\hline & 0.006 & 0.007 & 0.005 & 0.005 & 0.009 & 0.005 & 0.006 & 0.004 & 9.2 & 0.000 \\
\hline NGC 4486B & 0.084 & 0.082 & 0.046 & 0.076 & 0.172 & 0.079 & 0.072 & 0.075 & 188.6 & 0.000 \\
\hline & 0.004 & 0.006 & 0.004 & 0.004 & 0.009 & 0.003 & 0.004 & 0.003 & 3.1 & 0.000 \\
\hline NGC 4489 & 0.085 & 0.076 & 0.087 & 0.087 & 0.111 & 0.079 & 0.071 & 0.075 & 50.5 & 0.000 \\
\hline & 0.005 & 0.007 & 0.005 & 0.004 & 0.009 & 0.004 & 0.005 & 0.003 & 3.5 & 0.000 \\
\hline
\end{tabular}


P. Sánchez-Blázquez et al.: Stellar populations of early-type galaxies in different environments. I., Online Material p 16

Table D.3. continued.

\begin{tabular}{|c|c|c|c|c|c|c|c|c|c|c|}
\hline $\begin{array}{l}\text { Galaxy } \\
\text { name }\end{array}$ & $\begin{array}{c}\text { Fe4531' } \\
\text { (mag) }\end{array}$ & $\begin{array}{r}\text { C4668' } \\
\text { (mag) }\end{array}$ & $\begin{array}{c}\mathrm{H} \beta^{\prime} \\
(\mathrm{mag})\end{array}$ & $\begin{array}{c}\text { Fe5015' } \\
\text { (mag) }\end{array}$ & $\begin{array}{l}\mathrm{Mgb}^{\prime} \\
(\mathrm{mag})\end{array}$ & $\begin{array}{c}\text { Fe5270' } \\
\text { (mag) }\end{array}$ & $\begin{array}{c}\text { Fe5335' } \\
\text { (mag) }\end{array}$ & $\begin{array}{l}<\mathrm{Fe}>^{\prime} \\
(\mathrm{mag})\end{array}$ & $\begin{array}{c}\sigma \\
\left(\mathrm{km} \mathrm{s}^{-1}\right)\end{array}$ & $\begin{array}{c}E W([\mathrm{O} \text { III }]) \\
(\AA)\end{array}$ \\
\hline \multirow[t]{2}{*}{ NGC 4552} & 0.085 & 0.098 & 0.051 & 0.080 & 0.172 & 0.077 & 0.074 & 0.076 & 262.5 & 0.000 \\
\hline & 0.002 & 0.006 & 0.002 & 0.003 & 0.008 & 0.003 & 0.003 & 0.002 & 3.2 & 0.000 \\
\hline \multirow[t]{2}{*}{ NGC 4636} & 0.087 & 0.094 & 0.053 & 0.079 & 0.175 & 0.083 & 0.067 & 0.075 & 226.1 & -0.508 \\
\hline & 0.004 & 0.006 & 0.004 & 0.004 & 0.009 & 0.004 & 0.004 & 0.003 & 5.9 & 0.016 \\
\hline \multirow[t]{2}{*}{ NGC 4649} & 0.090 & 0.113 & 0.050 & 0.091 & 0.196 & 0.083 & 0.072 & 0.077 & 368.5 & 0.000 \\
\hline & 0.003 & 0.006 & 0.003 & 0.004 & 0.009 & 0.003 & 0.005 & 0.003 & 8.9 & 0.000 \\
\hline \multirow[t]{2}{*}{ NGC 4365} & 0.088 & 0.105 & 0.059 & 0.086 & 0.181 & 0.091 & 0.089 & 0.090 & 257.8 & 0.000 \\
\hline & 0.002 & 0.003 & 0.002 & 0.002 & 0.009 & 0.004 & 0.005 & 0.003 & 1.7 & 0.000 \\
\hline \multirow[t]{2}{*}{ NGC 4692} & 0.086 & 0.100 & 0.059 & 0.082 & 0.169 & 0.090 & 0.090 & 0.090 & 233.2 & 0.000 \\
\hline & 0.007 & 0.008 & 0.004 & 0.003 & 0.009 & 0.004 & 0.004 & 0.003 & 3.9 & 0.000 \\
\hline \multirow[t]{2}{*}{ NGC 4415} & 0.074 & 0.042 & 0.069 & 0.060 & 0.107 & 0.073 & 0.056 & 0.064 & 57.3 & 0.000 \\
\hline & 0.003 & 0.004 & 0.004 & 0.003 & 0.008 & 0.006 & 0.006 & 0.004 & 4.1 & 0.000 \\
\hline \multirow[t]{2}{*}{ NGC 4464} & 0.080 & 0.060 & 0.058 & 0.066 & 0.137 & 0.078 & 0.062 & 0.070 & 138.6 & -0.135 \\
\hline & 0.002 & 0.003 & 0.002 & 0.002 & 0.008 & 0.003 & 0.003 & 0.002 & 1.4 & 0.028 \\
\hline \multirow[t]{2}{*}{ NGC 4467} & 0.076 & 0.076 & 0.059 & 0.072 & 0.137 & 0.086 & 0.062 & 0.074 & 65.8 & 0.000 \\
\hline & 0.004 & 0.004 & 0.004 & 0.004 & 0.009 & 0.004 & 0.005 & 0.003 & 1.7 & 0.000 \\
\hline \multirow[t]{2}{*}{ NGC 4472} & 0.089 & 0.114 & 0.053 & 0.086 & 0.159 & 0.089 & 0.088 & 0.088 & 309.7 & 0.000 \\
\hline & 0.003 & 0.004 & 0.003 & 0.003 & 0.009 & 0.002 & 0.002 & 0.002 & 1.5 & 0.000 \\
\hline \multirow[t]{2}{*}{ NGC 4478} & 0.083 & 0.076 & 0.065 & 0.070 & 0.121 & 0.085 & 0.079 & 0.082 & 153.0 & 0.000 \\
\hline & 0.002 & 0.003 & 0.002 & 0.002 & 0.008 & 0.002 & 0.001 & 0.001 & 1.2 & 0.000 \\
\hline \multirow[t]{2}{*}{ NGC 4564} & 0.091 & 0.112 & 0.060 & 0.083 & 0.167 & 0.085 & 0.079 & 0.082 & 171.1 & 0.000 \\
\hline & 0.001 & 0.003 & 0.002 & 0.002 & 0.008 & 0.004 & 0.005 & 0.003 & 0.8 & 0.000 \\
\hline \multirow[t]{2}{*}{ NGC 4594} & 0.087 & 0.096 & 0.056 & 0.067 & 0.155 & 0.312 & 0.312 & 0.312 & 259.1 & -1.226 \\
\hline & 0.005 & 0.005 & 0.005 & 0.005 & 0.010 & 0.361 & 0.361 & 0.256 & 4.3 & 0.029 \\
\hline \multirow[t]{2}{*}{ NGC 4621} & 0.084 & 0.098 & 0.054 & 0.076 & 0.169 & 0.096 & 0.084 & 0.090 & 232.0 & 0.000 \\
\hline & 0.002 & 0.003 & 0.002 & 0.002 & 0.008 & 0.004 & 0.004 & 0.003 & 2.1 & 0.000 \\
\hline \multirow[t]{2}{*}{ NGC 4673} & 0.082 & 0.090 & 0.073 & 0.070 & 0.148 & 0.083 & 0.078 & 0.081 & 235.3 & -0.268 \\
\hline & 0.003 & 0.006 & 0.002 & 0.002 & 0.008 & 0.003 & 0.003 & 0.002 & 1.9 & 0.019 \\
\hline \multirow[t]{2}{*}{ NGC 4697} & 0.089 & 0.097 & 0.066 & 0.078 & 0.130 & 0.092 & 0.083 & 0.087 & 171.7 & -0.310 \\
\hline & 0.001 & 0.003 & 0.002 & 0.002 & 0.008 & 0.002 & 0.002 & 0.002 & 1.5 & 0.017 \\
\hline NGC 4742 & 0.070 & 0.064 & 0.130 & 0.067 & 0.080 & 0.071 & 0.055 & 0.063 & 91.9 & -0.133 \\
\hline & 0.001 & 0.003 & 0.002 & 0.002 & 0.008 & 0.005 & 0.005 & 0.004 & 0.5 & 0.018 \\
\hline NGC 4839 & 0.084 & 0.085 & 0.059 & 0.070 & 0.183 & 0.093 & 0.102 & 0.098 & 333.2 & 0.000 \\
\hline & 0.008 & 0.009 & 0.006 & 0.006 & 0.011 & 0.007 & 0.009 & 0.006 & 15.1 & 0.000 \\
\hline NGC 4842A & 0.085 & 0.091 & 0.056 & 0.077 & 0.174 & 0.095 & 0.089 & 0.092 & 232.2 & -0.397 \\
\hline & 0.005 & 0.007 & 0.004 & 0.004 & 0.009 & 0.004 & 0.005 & 0.003 & 3.4 & 0.019 \\
\hline NGC 4842B & 0.073 & 0.085 & 0.058 & 0.073 & 0.157 & 0.081 & 0.083 & 0.082 & 180.7 & 0.000 \\
\hline & 0.006 & 0.008 & 0.005 & 0.004 & 0.010 & 0.005 & 0.005 & 0.004 & 5.0 & 0.000 \\
\hline NGC 4864 & 0.081 & 0.072 & 0.061 & 0.076 & 0.151 & 0.091 & 0.097 & 0.094 & 220.6 & 0.000 \\
\hline & 0.006 & 0.007 & 0.005 & 0.005 & 0.010 & 0.005 & 0.006 & 0.004 & 5.0 & 0.000 \\
\hline NGC 4865 & 0.088 & 0.091 & 0.064 & 0.080 & 0.167 & 0.088 & 0.084 & 0.086 & 307.9 & 0.000 \\
\hline & 0.003 & 0.005 & 0.003 & 0.003 & 0.009 & 0.003 & 0.004 & 0.002 & 4.0 & 0.000 \\
\hline NGC 4867 & 0.075 & 0.094 & 0.067 & 0.078 & 0.165 & 0.079 & 0.082 & 0.080 & 246.9 & 0.000 \\
\hline & 0.005 & 0.005 & 0.005 & 0.005 & 0.010 & 0.005 & 0.006 & 0.004 & 5.5 & 0.000 \\
\hline NGC 4874 & 0.082 & 0.091 & 0.058 & 0.079 & 0.165 & 0.087 & 0.082 & 0.085 & 313.0 & 0.000 \\
\hline & 0.006 & 0.008 & 0.004 & 0.005 & 0.010 & 0.005 & 0.006 & 0.004 & 5.9 & 0.000 \\
\hline NGC 4875 & 0.074 & 0.070 & 0.059 & 0.069 & 0.160 & 0.081 & 0.074 & 0.077 & 189.6 & 0.000 \\
\hline & 0.007 & 0.008 & 0.004 & 0.004 & 0.009 & 0.004 & 0.004 & 0.003 & 4.0 & 0.000 \\
\hline NGC 4889 & 0.091 & 0.106 & 0.052 & 0.086 & 0.188 & 0.088 & 0.080 & 0.084 & 350.0 & 0.000 \\
\hline & 0.007 & 0.006 & 0.006 & 0.006 & 0.009 & 0.005 & 0.006 & 0.004 & 4.2 & 0.000 \\
\hline NGC 4908 & 0.086 & 0.069 & 0.058 & 0.069 & 0.152 & 0.076 & 0.073 & 0.074 & 201.5 & 0.000 \\
\hline & 0.006 & 0.008 & 0.004 & 0.004 & 0.009 & 0.004 & 0.004 & 0.003 & 2.4 & 0.000 \\
\hline NGC 5638 & 0.085 & 0.090 & 0.070 & 0.071 & 0.137 & 0.088 & 0.076 & 0.082 & 159.4 & -0.426 \\
\hline & 0.002 & 0.003 & 0.002 & 0.002 & 0.008 & 0.002 & 0.001 & 0.001 & 1.3 & 0.019 \\
\hline NGC 5796 & 0.090 & 0.112 & 0.064 & 0.079 & 0.172 & 0.076 & 0.072 & 0.074 & 273.6 & -0.428 \\
\hline & 0.003 & 0.004 & 0.003 & 0.003 & 0.018 & 0.012 & 0.016 & 0.010 & 2.5 & 0.018 \\
\hline
\end{tabular}


Table D.3. continued.

\begin{tabular}{|c|c|c|c|c|c|c|c|c|c|c|}
\hline $\begin{array}{l}\text { Galaxy } \\
\text { name }\end{array}$ & $\begin{array}{c}\begin{array}{c}\text { Fe4531' } \\
\text { (mag) }\end{array}\end{array}$ & $\begin{array}{l}\text { C4668' } \\
\text { (mag) }\end{array}$ & $\begin{array}{l}\mathrm{H} \beta^{\prime} \\
(\mathrm{mag})\end{array}$ & $\begin{array}{c}\text { Fe5015' } \\
\text { (mag) }\end{array}$ & $\begin{array}{l}\mathrm{Mgb}^{\prime} \\
\text { (mag) }\end{array}$ & $\begin{array}{c}\text { Fe5270' } \\
\text { (mag) }\end{array}$ & $\begin{array}{c}\text { Fe5335' } \\
\text { (mag) }\end{array}$ & $\begin{array}{l}<\mathrm{Fe}>^{\prime} \\
(\mathrm{mag})\end{array}$ & $\begin{array}{c}\sigma \\
\left(\mathrm{km} \mathrm{s}^{-1}\right)\end{array}$ & $\begin{array}{c}E W([\mathrm{O} \text { III }]) \\
(\AA)\end{array}$ \\
\hline \multirow[t]{2}{*}{ NGC 5812} & 0.090 & 0.111 & 0.065 & 0.083 & 0.154 & 0.089 & 0.088 & 0.088 & 216.8 & 0.000 \\
\hline & 0.002 & 0.003 & 0.002 & 0.002 & 0.008 & 0.002 & 0.001 & 0.001 & 1.3 & 0.000 \\
\hline \multirow{2}{*}{ NGC 5813} & 0.085 & 0.087 & 0.066 & 0.067 & 0.152 & 0.083 & 0.074 & 0.079 & 257.1 & -1.026 \\
\hline & 0.002 & 0.003 & 0.002 & 0.002 & 0.008 & 0.002 & 0.002 & 0.001 & 1.4 & 0.019 \\
\hline \multirow{2}{*}{ NGC 5831} & 0.083 & 0.096 & 0.077 & 0.077 & 0.131 & 0.094 & 0.085 & 0.089 & 163.1 & -0.438 \\
\hline & 0.002 & 0.003 & 0.002 & 0.002 & 0.008 & 0.002 & 0.001 & 0.001 & 1.0 & 0.018 \\
\hline \multirow[t]{2}{*}{ NGC 5845} & 0.085 & 0.095 & 0.060 & 0.075 & 0.137 & 0.099 & 0.067 & 0.083 & 253.7 & 0.000 \\
\hline & 0.001 & 0.003 & 0.002 & 0.002 & 0.008 & 0.010 & 0.013 & 0.008 & 1.3 & 0.000 \\
\hline \multirow[t]{2}{*}{ NGC 5846} & 0.088 & 0.101 & 0.058 & 0.071 & 0.161 & 0.085 & 0.079 & 0.082 & 248.0 & -0.537 \\
\hline & 0.002 & 0.003 & 0.003 & 0.003 & 0.009 & 0.002 & 0.001 & 0.001 & 1.9 & 0.016 \\
\hline \multirow[t]{2}{*}{ NGC 5846A } & 0.082 & 0.085 & 0.055 & 0.068 & 0.156 & 0.078 & 0.074 & 0.076 & 164.1 & 0.000 \\
\hline & 0.002 & 0.003 & 0.003 & 0.003 & 0.009 & 0.003 & 0.002 & 0.002 & 1.2 & 0.000 \\
\hline \multirow[t]{2}{*}{ NGC 6127} & 0.089 & 0.103 & 0.057 & 0.084 & 0.178 & 0.084 & 0.079 & 0.081 & 251.3 & 0.000 \\
\hline & 0.002 & 0.003 & 0.003 & 0.004 & 0.012 & 0.002 & 0.002 & 0.002 & 1.8 & 0.016 \\
\hline \multirow[t]{2}{*}{ NGC 6166} & 0.068 & 0.089 & 0.036 & 0.051 & 0.201 & 0.091 & 0.056 & 0.073 & 333.7 & -2.821 \\
\hline & 0.017 & 0.013 & 0.015 & 0.014 & 0.013 & 0.010 & 0.013 & 0.008 & 15.0 & 0.019 \\
\hline \multirow[t]{2}{*}{ NGC 6411} & 0.091 & 0.090 & 0.069 & 0.081 & 0.143 & 0.085 & 0.084 & 0.084 & 183.7 & 0.000 \\
\hline & 0.003 & 0.004 & 0.003 & 0.003 & 0.008 & 0.003 & 0.003 & 0.002 & 3.5 & 0.000 \\
\hline \multirow[t]{2}{*}{ NGC 6482} & 0.092 & 0.115 & 0.036 & 0.082 & 0.192 & 0.091 & 0.089 & 0.090 & 303.3 & -0.276 \\
\hline & 0.003 & 0.008 & 0.003 & 0.003 & 0.009 & 0.003 & 0.004 & 0.002 & 3.3 & 0.018 \\
\hline \multirow[t]{2}{*}{ NGC 6577} & 0.092 & 0.106 & 0.054 & 0.066 & 0.173 & 0.090 & 0.084 & 0.087 & 269.2 & 0.000 \\
\hline & 0.004 & 0.004 & 0.004 & 0.003 & 0.009 & 0.003 & 0.004 & 0.003 & 4.1 & 0.000 \\
\hline \multirow[t]{2}{*}{ NGC 6702} & 0.085 & 0.104 & 0.087 & 0.089 & 0.181 & 0.090 & 0.083 & 0.087 & 167.5 & -0.287 \\
\hline & 0.003 & 0.003 & 0.003 & 0.004 & 0.012 & 0.002 & 0.001 & 0.001 & 1.7 & 0.019 \\
\hline \multirow[t]{2}{*}{ NGC 6703} & 0.087 & 0.103 & 0.070 & 0.076 & 0.114 & 0.087 & 0.079 & 0.083 & 190.7 & -0.314 \\
\hline & 0.002 & 0.003 & 0.002 & 0.002 & 0.008 & 0.002 & 0.001 & 0.001 & 1.2 & 0.019 \\
\hline NGC 7052 & 0.085 & 0.095 & 0.028 & 0.065 & 0.173 & 0.076 & 0.063 & 0.069 & 201.5 & -0.864 \\
\hline & 0.004 & 0.004 & 0.004 & 0.003 & 0.009 & 0.004 & 0.004 & 0.003 & 6.2 & 0.018 \\
\hline IC 767 & 0.065 & 0.034 & 0.104 & 0.063 & 0.064 & 0.050 & 0.048 & 0.049 & 40.0 & 0.000 \\
\hline & 0.007 & 0.007 & 0.007 & 0.009 & 0.010 & 0.008 & 0.014 & 0.008 & 7.2 & 0.000 \\
\hline IC 794 & 0.081 & 0.065 & 0.077 & 0.066 & 0.083 & 0.074 & 0.076 & 0.075 & 36.9 & 0.000 \\
\hline & 0.006 & 0.005 & 0.006 & 0.005 & 0.009 & 0.006 & 0.006 & 0.005 & 4.8 & 0.000 \\
\hline IC 832 & 0.091 & 0.068 & 0.067 & 0.062 & 0.137 & 0.075 & 0.069 & 0.072 & 175.3 & -0.192 \\
\hline & 0.003 & 0.007 & 0.004 & 0.003 & 0.009 & 0.004 & 0.004 & 0.003 & 3.3 & 0.018 \\
\hline IC 3957 & 0.080 & 0.074 & 0.058 & 0.071 & 0.158 & 0.074 & 0.075 & 0.074 & 171.2 & 0.000 \\
\hline & 0.004 & 0.006 & 0.004 & 0.003 & 0.009 & 0.004 & 0.004 & 0.003 & 3.2 & 0.000 \\
\hline IC 3959 & 0.083 & 0.089 & 0.064 & 0.076 & 0.174 & 0.083 & 0.079 & 0.081 & 226.1 & 0.000 \\
\hline & 0.004 & 0.007 & 0.003 & 0.003 & 0.009 & 0.003 & 0.003 & 0.002 & 2.4 & 0.000 \\
\hline IC 3963 & 0.085 & 0.069 & 0.070 & 0.071 & 0.139 & 0.084 & 0.074 & 0.079 & 130.3 & 0.000 \\
\hline & 0.005 & 0.007 & 0.005 & 0.004 & 0.009 & 0.005 & 0.005 & 0.003 & 4.0 & 0.000 \\
\hline IC 3973 & 0.083 & 0.100 & 0.073 & 0.077 & 0.159 & 0.082 & 0.083 & 0.082 & 256.3 & 0.000 \\
\hline & 0.003 & 0.004 & 0.004 & 0.003 & 0.009 & 0.003 & 0.004 & 0.002 & 3.1 & 0.000 \\
\hline IC 4026 & 0.074 & 0.082 & 0.068 & 0.076 & 0.143 & 0.093 & 0.076 & 0.084 & 58.3 & 0.000 \\
\hline & 0.008 & 0.009 & 0.006 & 0.005 & 0.010 & 0.005 & 0.005 & 0.004 & 15.0 & 0.000 \\
\hline IC 4042 & 0.055 & 0.043 & 0.041 & 0.071 & 0.155 & 0.079 & 0.066 & 0.072 & 170.9 & -0.133 \\
\hline & 0.005 & 0.005 & 0.004 & 0.004 & 0.009 & 0.004 & 0.004 & 0.003 & 4.4 & 0.018 \\
\hline IC 4051 & 0.094 & 0.106 & 0.051 & 0.076 & 0.192 & 0.087 & 0.083 & 0.085 & 298.8 & 0.000 \\
\hline & 0.005 & 0.005 & 0.005 & 0.004 & 0.010 & 0.005 & 0.006 & 0.004 & 7.8 & 0.000 \\
\hline CGCG 159-041 & 0.078 & 0.102 & 0.050 & 0.066 & 0.173 & 0.079 & 0.069 & 0.074 & 189.6 & 0.000 \\
\hline & 0.004 & 0.006 & 0.003 & 0.003 & 0.009 & 0.004 & 0.004 & 0.003 & 2.9 & 0.000 \\
\hline CGCG 159-043 & 0.084 & 0.099 & 0.054 & 0.055 & 0.174 & 0.081 & 0.072 & 0.077 & 231.2 & -0.455 \\
\hline & 0.004 & 0.006 & 0.003 & 0.003 & 0.009 & 0.004 & 0.004 & 0.003 & 2.7 & 0.018 \\
\hline CGCG 159-083 & 0.073 & 0.079 & 0.034 & 0.066 & 0.145 & 0.076 & 0.073 & 0.075 & 212.9 & 0.000 \\
\hline & 0.004 & 0.006 & 0.004 & 0.003 & 0.009 & 0.004 & 0.004 & 0.003 & 4.7 & 0.000 \\
\hline CGCG 159-089 & 0.084 & 0.082 & 0.075 & 0.076 & 0.135 & 0.085 & 0.076 & 0.081 & 141.0 & 0.000 \\
\hline & 0.006 & 0.008 & 0.004 & 0.003 & 0.009 & 0.004 & 0.004 & 0.003 & 2.6 & 0.000 \\
\hline DRCG 27-032 & 0.086 & 0.057 & 0.052 & 0.053 & 0.128 & 0.080 & 0.076 & 0.078 & 104.5 & 0.000 \\
\hline & 0.012 & 0.011 & 0.011 & 0.009 & 0.014 & 0.010 & 0.011 & 0.007 & 18.7 & 0.000 \\
\hline DRCG 27-127 & 0.077 & 0.066 & 0.069 & 0.063 & 0.156 & 0.088 & 0.084 & 0.086 & 102.5 & 0.000 \\
\hline & 0.008 & 0.009 & 0.006 & 0.005 & 0.010 & 0.006 & 0.006 & 0.004 & 10.9 & 0.000 \\
\hline
\end{tabular}


P. Sánchez-Blázquez et al.: Stellar populations of early-type galaxies in different environments. I., Online Material p 18

Table D.3. continued.

\begin{tabular}{|c|c|c|c|c|c|c|c|c|c|c|}
\hline $\begin{array}{c}\text { Galaxy } \\
\text { name }\end{array}$ & $\begin{array}{c}\text { Fe4531' } \\
\text { (mag) }\end{array}$ & $\begin{array}{l}\text { C4668' } \\
\text { (mag) }\end{array}$ & $\begin{array}{c}\mathrm{H} \beta^{\prime} \\
(\mathrm{mag})\end{array}$ & $\begin{array}{c}\text { Fe5015' } \\
\text { (mag) }\end{array}$ & $\begin{array}{l}\mathrm{Mgb}^{\prime} \\
(\mathrm{mag})\end{array}$ & $\begin{array}{c}\begin{array}{c}\text { Fe5270' } \\
(\mathrm{mag})\end{array}\end{array}$ & $\begin{array}{c}\begin{array}{c}\text { Fe5335' } \\
(\mathrm{mag})\end{array}\end{array}$ & $\begin{array}{l}<\mathrm{Fe}>^{\prime} \\
(\mathrm{mag})\end{array}$ & $\begin{array}{c}\sigma \\
\left(\mathrm{km} \mathrm{s}^{-1}\right)\end{array}$ & $\begin{array}{c}E W\left(\left[\begin{array}{l}\text { III }]) \\
(\AA)\end{array}\right.\right. \\
(\AA)\end{array}$ \\
\hline \multirow[t]{2}{*}{$\overline{\text { DRCG 27-128 }}$} & 0.083 & 0.052 & 0.075 & 0.065 & 0.131 & 0.076 & 0.069 & 0.072 & 99.3 & 0.000 \\
\hline & 0.008 & 0.009 & 0.005 & 0.005 & 0.009 & 0.005 & 0.005 & 0.004 & 7.8 & 0.000 \\
\hline \multirow[t]{2}{*}{ GMP 2585} & 0.053 & 0.020 & 0.077 & 0.052 & 0.080 & 0.068 & 0.046 & 0.057 & 28.4 & 0.000 \\
\hline & 0.010 & 0.010 & 0.010 & 0.008 & 0.012 & 0.009 & 0.010 & 0.007 & 7.0 & 0.000 \\
\hline \multirow[t]{2}{*}{ GMP 3121} & 0.070 & 0.057 & 0.059 & 0.070 & 0.108 & 0.070 & 0.078 & 0.074 & 40.0 & 0.000 \\
\hline & 0.011 & 0.011 & 0.010 & 0.008 & 0.013 & 0.010 & 0.011 & 0.007 & 10.0 & 0.000 \\
\hline \multirow[t]{2}{*}{ GMP 3131} & 0.054 & 0.021 & 0.058 & 0.045 & 0.088 & 0.072 & 0.049 & 0.061 & 40.0 & 0.000 \\
\hline & 0.012 & 0.011 & 0.011 & 0.009 & 0.014 & 0.010 & 0.011 & 0.008 & 10.0 & 0.000 \\
\hline \multirow{2}{*}{ GMP 3196} & 0.068 & 0.028 & 0.068 & 0.056 & 0.106 & 0.058 & 0.056 & 0.057 & 51.7 & 0.000 \\
\hline & 0.007 & 0.008 & 0.007 & 0.006 & 0.010 & 0.006 & 0.007 & 0.005 & 7.2 & 0.000 \\
\hline \multirow[t]{2}{*}{$M G C+5-31-63$} & 0.075 & 0.046 & 0.058 & 0.071 & 0.139 & 0.074 & 0.067 & 0.070 & 155.1 & 0.000 \\
\hline & 0.008 & 0.009 & 0.005 & 0.005 & 0.010 & 0.006 & 0.006 & 0.004 & 9.8 & 0.000 \\
\hline \multirow[t]{2}{*}{ PGC 126756} & 0.061 & 0.037 & 0.069 & 0.057 & 0.084 & 0.063 & 0.044 & 0.053 & 40.0 & 0.000 \\
\hline & 0.010 & 0.010 & 0.008 & 0.007 & 0.011 & 0.008 & 0.009 & 0.006 & 10.0 & 0.000 \\
\hline \multirow[t]{2}{*}{ PGC 126775} & 0.082 & 0.038 & 0.099 & 0.050 & 0.074 & 0.058 & 0.044 & 0.051 & 40.0 & 0.000 \\
\hline & 0.011 & 0.009 & 0.010 & 0.008 & 0.013 & 0.009 & 0.011 & 0.007 & 10.0 & 0.000 \\
\hline \multirow[t]{2}{*}{ RB 091} & 0.089 & 0.071 & 0.064 & 0.072 & 0.147 & 0.076 & 0.074 & 0.075 & 88.0 & 0.000 \\
\hline & 0.009 & 0.009 & 0.006 & 0.005 & 0.010 & 0.006 & 0.006 & 0.004 & 12.0 & 0.000 \\
\hline
\end{tabular}

